sid.inpe.br/plutao/2014/11.16.19.25-TDI

\title{
DRAINAGE NETWORKS AND WATERSHEDS \\ DELINEATION DERIVED FROM TIN-BASED DIGITAL ELEVATION MODELS
}

\begin{abstract}
Henrique Rennó de Azeredo Freitas
Master's Dissertation of the Graduate Course in Applied Computing, advised by Drs. Corina da Costa Freitas, Sergio Rosim e João Ricardo de Freitas Oliveira, approved in October $2^{\text {nd }}$, 2014 .
\end{abstract}

URL of the original document:

<http://urlib.net/8JMKD3MGP3W/3HDPRRS >

INPE

São José dos Campos

2014 


\section{PUBLISHED BY:}

Instituto Nacional de Pesquisas Espaciais - INPE

Gabinete do Diretor (GB)

Serviço de Informação e Documentação (SID)

Caixa Postal 515 - CEP 12.245-970

São José dos Campos - SP - Brasil

Tel.:(012) 3208-6923/6921

Fax: (012) 3208-6919

E-mail: pubtc@sid.inpe.br

\section{BOARD OF PUBLISHING AND PRESERVATION OF INPE INTELLECTUAL PRODUCTION (RE/DIR-204):}

\section{Chairperson:}

Marciana Leite Ribeiro - Serviço de Informação e Documentação (SID)

\section{Members:}

Dr. Gerald Jean Francis Banon - Coordenação Observação da Terra (OBT)

Dr. Amauri Silva Montes - Coordenação Engenharia e Tecnologia Espaciais (ETE)

Dr. André de Castro Milone - Coordenação Ciências Espaciais e Atmosféricas (CEA)

Dr. Joaquim José Barroso de Castro - Centro de Tecnologias Espaciais (CTE)

Dr. Manoel Alonso Gan - Centro de Previsão de Tempo e Estudos Climáticos $(\mathrm{CPT})$

Dra Maria do Carmo de Andrade Nono - Conselho de Pós-Graduação

Dr. Plínio Carlos Alvalá - Centro de Ciência do Sistema Terrestre (CST)

\section{DIGITAL LIBRARY:}

Dr. Gerald Jean Francis Banon - Coordenação de Observação da Terra (OBT)

\section{DOCUMENT REVIEW:}

Maria Tereza Smith de Brito - Serviço de Informação e Documentação (SID)

Yolanda Ribeiro da Silva Souza - Serviço de Informação e Documentação (SID)

\section{ELECTRONIC EDITING:}

Maria Tereza Smith de Brito - Serviço de Informação e Documentação (SID)

André Luis Dias Fernandes - Serviço de Informação e Documentação (SID) 
sid.inpe.br/plutao/2014/11.16.19.25-TDI

\title{
DRAINAGE NETWORKS AND WATERSHEDS \\ DELINEATION DERIVED FROM TIN-BASED DIGITAL ELEVATION MODELS
}

\begin{abstract}
Henrique Rennó de Azeredo Freitas
Master's Dissertation of the Graduate Course in Applied Computing, advised by Drs. Corina da Costa Freitas, Sergio Rosim e João Ricardo de Freitas Oliveira, approved in October $2^{\text {nd }}$, 2014 .
\end{abstract}

URL of the original document:

<http://urlib.net/8JMKD3MGP3W/3HDPRRS >

INPE

São José dos Campos

2014 
Freitas, Henrique Rennó de Azeredo.

F884d Drainage networks and watersheds delineation derived from TIN-based digital elevation models / Henrique Rennó de Azeredo Freitas. - São José dos Campos : INPE, 2014.

xxii + 93 p. ; (sid.inpe.br/plutao/2014/11.16.19.25-TDI)

Dissertation (Master's in Applied Computing) - Instituto Nacional de Pesquisas Espaciais, São José dos Campos, 2014.

Advisors : Drs. Corina da Costa Freitas, Sergio Rosim, and João Ricardo de Freitas Oliveira.

1. Digital elevation models 2. Triangulated irregular networks (TIN) 3. Flats (landforms). 4. Pits. 5. Drainage. I.Title.

\section{(c) (7) (8)}

Esta obra foi licenciada sob uma Licença Creative Commons Atribuição-NãoComercial 3.0 Não Adaptada.

This work is licensed under a Creative Commons Attribution-NonCommercial 3.0 Unported License. 
Aprovado (a) pela Banca Examinadora em cumprimento ao requisito exigido para obtenção do Título de Mestre em

Computação Aplicada

Dra. Lúbia Vinhas

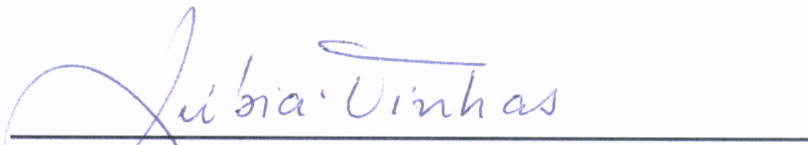

Presidente / INPE / São José dos Campos - SP

Dra. Corina da Costa Freitas

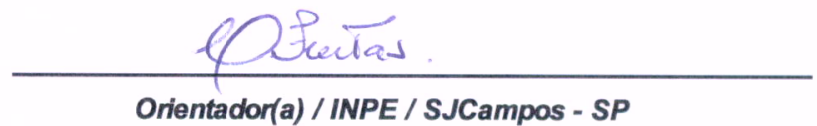

Dr. Sergio Rosim

Dr. João Ricardo de Freitas Oliveira

Dr. Rafael Duarte Coelho dos Santos
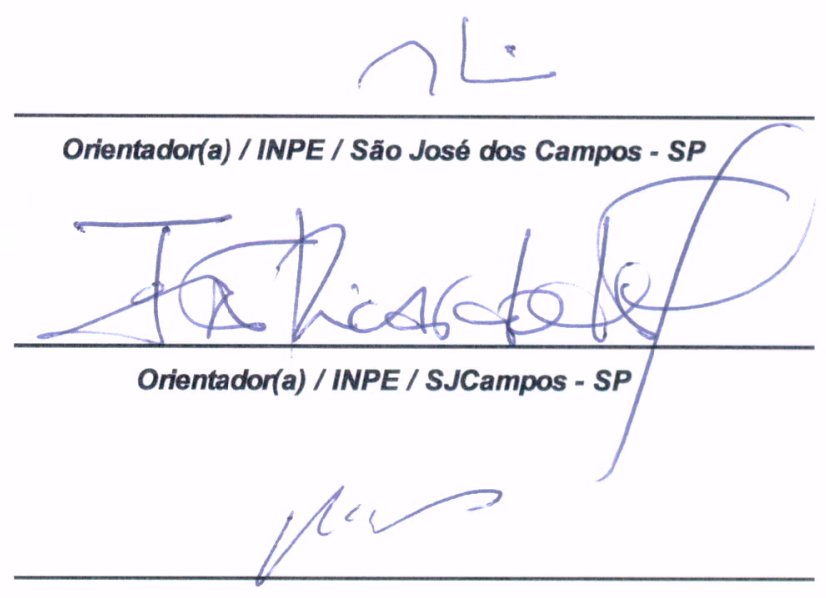

Membro da Banca / INPE / SJCampos - SP

Dr. Wilson Cabral de Sousa Júnior

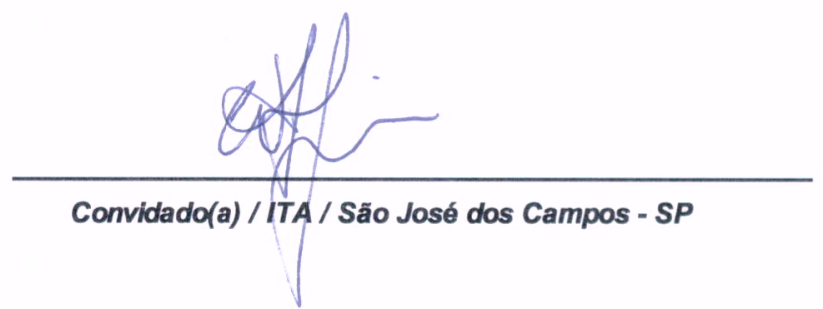

Este trabalho fol aprovado por:

( ) maioria simples

(X) unanimidade 

"Life is an opportunity, benefit from it.

Life is beauty, admire it.

Life is a dream, realize it.

Life is a challenge, meet it.

Life is a duty, complete it.

Life is a game, play it.

Life is a promise, fulfill it.

Life is sorrow, overcome it.

Life is a song, sing it.

Life is a struggle, accept it.

Life is a tragedy, confront it.

Life is an adventure, dare it.

Life is luck, make it.

Life is too precious, do not destroy it.

Life is life, fight for it."

\section{Mother Teresa}

"Music is the one incorporeal entrance into the higher world of knowledge which comprehends mankind but which mankind cannot comprehend."

LudWig VAN BeEthoven 

So my parents Senita and Pramon 



\section{ACKNOWLEDGEMENTS}

I am deeply thankful to my parents who have always supported me.

I gratefully thank to all my friends for every good moment in my life.

I am sincerely grateful to my advisors for their friendship and for the opportunity to develop this work.

I also thank to CAPES for the financial support. 



\begin{abstract}
Digital Elevation Models (DEMs) are objects of great importance in many studies of natural sciences and one relevant area of their application is hydrology, as from a DEM it is possible to generate drainage networks and watersheds, which are essential elements in the understanding of hydrological processes. All the methods developed to generate drainage networks from a DEM depend on its representation and the most common representation is given by regular grids, where elevation values are assigned to cells uniformly distributed over space. However, this representation presents limitations in the flow directions, which are defined in only eight distinct directions. This work considers the terrain model given by a Triangulated Irregular Network (TIN) generated from contour lines and sampled points, where the terrain surface is modeled by several triangles defined from a set of points adequately distributed over space, so that the TIN can efficiently adapt to its irregularities. In addition, flow directions on TINs encompass all the possible directions and depend on the slope of the triangles, so this structure is more flexible regarding the calculation of water flows. The triangulation used in this work is the constrained Delaunay triangulation and the method that generates drainage networks considers flow directions given by the gradient of the plane defined from each triangle. Problems that commonly occur in DEMs such as flat areas and pits, which create inconsistencies in the terrain model and discontinuities in flows, also deserve special attention. In the present work, flat areas are removed by the insertion of new points into the triangulation with interpolated elevation values and pits by the definition of paths of points starting from the pit until reaching another point of lower elevation, where all these points have their elevation values re-interpolated. Most importantly, this work describes a methodology for calculating drainage paths from a TIN traced by processing the triangles with an associated priority and then connected defining a drainage graph structure, which is proposed in this work for the calculation of accumulated flows. Afterwards, drainage networks are generated according to given threshold values and watersheds are then delineated from the drainage networks. All the results from the TIN are qualitatively compared to regular grid results generated with the TerraHidro computational platform developed at INPE and also to an available drainage network produced by specialists and considered here as a reference of the main courses of water over the terrain. Results are very promising showing that TIN terrain models can be used to generate drainage networks from accumulated flows consistent with real-world hydrological patterns.
\end{abstract}





\title{
REDES DE DRENAGEM E DELIMITAÇÃO DE BACIAS HIDROGRÁFICAS DERIVADAS DE MODELOS DIGITAIS DE ELEVAÇÃO BASEADOS EM TIN
}

\begin{abstract}
RESUMO
Modelos Digitais de Elevação (MDEs) são objetos de grande importância em muitos estudos das ciências naturais e uma área relevante de sua aplicação é a hidrologia, pois a partir de um MDE é possível gerar redes de drenagem e bacias hidrográficas, as quais são elementos essenciais no entendimento de processos hidrológicos. Todos os métodos desenvolvidos para gerar redes de drenagem a partir de um MDE dependem da sua representação e a representação mais comum é dada por grades regulares, onde valores de elevação são atribuídos em células uniformemente distribuídas no espaço. Entretanto, essa representação apresenta limitações nas direções de fluxo, as quais são definidas em apenas oito direções distintas. Este trabalho considera o modelo de terreno dado por uma Rede Triangular Irregular (Triangulated Irregular Network ou TIN) gerada de curvas de nível e pontos amostrados, onde a superfície do terreno é modelada por vários triângulos definidos a partir de um conjunto de pontos adequadamente distribuídos no espaço, de forma que o TIN pode eficientemente se adaptar as suas irregularidades. Além disso, direções de fluxo sobre TINs abrangem todas as direções possíveis e dependem da declividade dos triângulos, de modo que essa estrutura é mais flexível ao se considerar o cálculo dos fluxos de água. A triangulação utilizada neste trabalho é a triangulação de Delaunay restrita e o método que gera redes de drenagem considera direções de fluxo dadas pelo gradiente do plano definido por cada triângulo. Problemas que comumente ocorrem em MDEs tais como áreas planas e fossos, os quais criam inconsistências no modelo de terreno e descontinuidades nos fluxos, também merecem atenção especial. No presente trabalho, áreas planas são removidas pela inserção de novos pontos na triangulação com valores de elevação interpolados e fossos pela definição de caminhos de pontos iniciando do fosso até alcançar um outro ponto de menor elevação, onde todos esses pontos têm seus valores de elevação reinterpolados. Sobretudo, este trabalho descreve uma metodologia para calcular caminhos de drenagem a partir de um TIN traçados ao processar os triângulos com uma prioridade associada e depois conectados definindo uma estrutura de grafo de drenagem, a qual é proposta neste trabalho para o cálculo de fluxos acumulados. Posteriormente, redes de drenagem são geradas de acordo com determinados valores de limiar e bacias hidrográficas são então delimitadas a partir das redes de drenagem. Todos os resultados do TIN são comparados qualitativamente a resultados de grade regular gerados com a plataforma computacional TerraHidro desenvolvida no INPE e também a uma rede de drenagem disponível, produzida por especialistas e considerada aqui como uma referência dos principais cursos d'água no terreno. Os resultados são bastante promissores mostrando que modelos de terreno TIN podem ser utilizados para gerar redes de drenagem a partir de fluxos acumulados consistentes com padrões hidrológicos reais.
\end{abstract}





\section{LIST OF FIGURES}

Page

1.1 Flowchart of the steps performed in this work . . . . . . . . . . 4

$2.13 \mathrm{D}$ view of a regular grid . . . . . . . . . . . . . . . 7

2.2 Contour lines and sampled points define elevation data . . . . . . . . . 9

2.3 Contour lines and sampled points inconsistencies . . . . . . . . . . . 11

$2.43 \mathrm{D}$ view of a TIN . . . . . . . . . . . . . . . . . . . 13

2.5 Connections of triangles edges may create terrain inconsistencies . . . . . 13

2.6 Delaunay triangulation criteria . . . . . . . . . . . . . . . 14

2.7 Enclosing triangle of the set of points . . . . . . . . . . . . . . 15

2.8 Two different cases of inserting a point into the triangulation . . . . . . . 16

2.9 Illegal edge . . . . . . . . . . . . . . . . . . . . . . . . 17

2.10 Rotating an edge maximizes interior angles of triangles . . . . . . . . . . 17

2.11 A new point $p_{r}$ locally changes the triangulation . . . . . . . . . . . 18

2.12 Tree structure reflects triangles divisions . . . . . . . . . . . . . . . . . . 19

2.13 Triangulations before and after removing intersections . . . . . . . . . . 19

2.14 Edges are rotated and intersections are removed . . . . . . . . . . . 21

2.15 TIN from contour lines with flat triangles . . . . . . . . . . . . . . . 22

2.16 Flat triangles and critical edges . . . . . . . . . . . . . 23

2.17 Paths of triangles initiated at corner triangles define critical points for interpolation . . . . . . . . . . . . . . . 25

2.18 Interpolation of critical points following branching paths . . . . . . 26

2.19 Two different cases where the interpolation of elevation values of critical points is not possible . . . . . . . . . . . . . . . . 27

2.20 Neighboring contour lines indicate how interpolation is performed . . . . 28

2.21 Connections between triangles edges created a pit in the TIN . . . . . . 29

2.22 Pit removal by lowering the elevation of some points in a breaching path 30

2.23 Removal of flat areas creates a pit . . . . . . . . . . . . . . . . . . . . . . . 31

2.24 Paths of critical points for pit removal . . . . . . . . . . . . . . 32

3.1 Accumulated flows over the regular grid . . . . . . . . . . . . . 35

3.2 Drainage network and watersheds defined from a regular grid . . . . . . 36

3.3 Vectors defined from a triangle . . . . . . . . . . . . . . . 37

3.4 In-In-Out and In-Out-Out flow cases . . . . . . . . . . . . . . . . 38

3.5 Trickle path of a point $p$ includes a cycle of terrain features . . . . . . . . 39

3.6 Strip map over the TIN . . . . . . . . . . . . . . . . . . . . . . . 40

3.7 Drainage path over the TIN . . . . . . . . . . . . . . . 42 
3.8 Flows over the TIN . . . . . . . . . . . . . . . . . . . 44

3.9 Current flow continues from a vertex across a triangle . . . . . . . . . 44

3.10 Triangles centroids represent the elevation of each triangle . . . . . . 45

3.11 Connection of drainage paths . . . . . . . . . . . . 46

3.12 Contributing areas from triangles in two different cases . . . . . . . 49

3.13 Nodes of the drainage graph have upstream and downstream nodes . . . 51

3.14 Accumulated flows defined by a topological sort of the nodes of the drainage graph . . . . . . . . . . . . . . 53

3.15 Flow segments included in a drainage network depend on different threshold values . . . . . . . . . . . . . . . . 56

3.16 Drainage networks generated from different threshold values . . . . . . 58

3.17 Watershed delineated from the flow segments of the drainage graph . . . 60

3.18 Drainage segments of a drainage network . . . . . . . . . . . . 61

3.19 Subwatersheds delineated from each drainage segment . . . . . . . . . 62

4.1 Image from the area of study $\ldots \ldots \ldots \ldots$

4.2 Reference drainage network from the 'Cidade Viva' database . . . . . . . 64

4.3 Drainage paths and reference drainage network . . . . . . . . . 65

4.4 Drainage paths over the TIN . . . . . . . . . . . 66

4.5 Comparison between drainage networks . . . . . . . . . . . . . 67

4.6 Watersheds and subwatersheds delineated from the TIN . . . . . . 68

4.7 Execution times for different number of input points . . . . . . . . 72

4.8 Drainage networks generated from the TIN and from the regular grid . . 73

4.9 Differences between the drainage networks from the TIN and from the

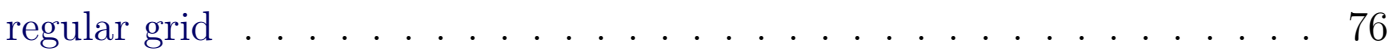

4.10 Watersheds and subwatersheds delineated from the TIN and from the

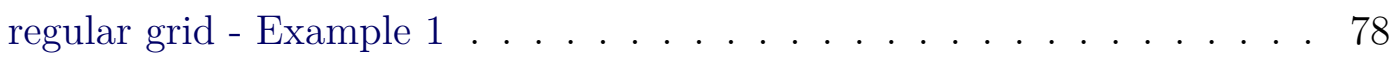

4.11 Differences between the subwatersheds from the TIN and from the regular grid . . . . . . . . . . . . . . . . . . . 79

4.12 Watersheds and subwatersheds delineated from the TIN and from the regular grid - Example $2 \ldots \ldots$. . . . . . . . . . . 80

4.13 Watersheds and subwatersheds delineated from the TIN and from the regular grid - Example $3 \ldots \ldots$. . . . . . . . . . . . 81 


\section{LIST OF TABLES}

$\underline{\text { Page }}$

2.1 Original and corrected sequences of contour line data . . . . . . . . . . . 12

2.2 Interpolated elevation values of critical points . . . . . . . . . 25

4.1 Output information . . . . . . . . . . . . . . . 70

4.2 TIN and regular grid computational times . . . . . . . . . . . 83 



\section{LIST OF ABBREVIATIONS}

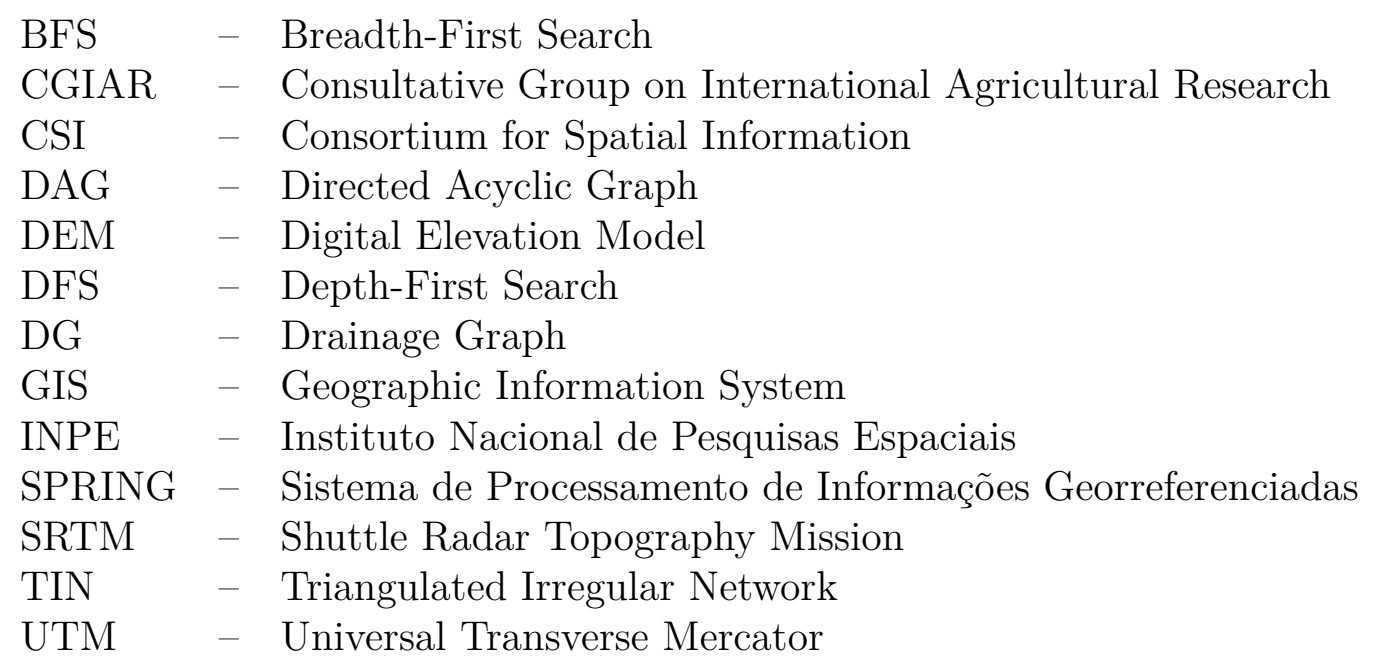





\section{CONTENTS}

$\underline{\text { Page }}$

1 INTRODUCTION ......................... 1

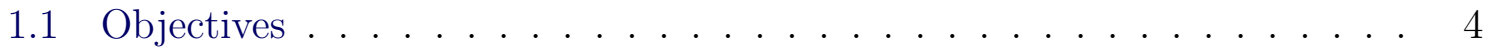

1.1.1 General objective . . . . . . . . . . . . . . . . . . . 4

1.1.2 Specific objectives ..................... 4

1.2 Contributions . . . . . . . . . . . . . . . . 5

1.3 Work organization . . . . . . . . . . . . . . . . . 6

2 DIGITAL ELEVATION MODELS . . . . . . . . . . 7

2.1 Regular grids . . . . . . . . . . . . . . . . . 7

2.2 Contour lines . . . . . . . . . . . . . . . . . . . . 8

2.2.1 Input data corrections . . . . . . . . . . . . . . . . . . . . 10

2.3 Triangulated irregular networks . . . . . . . . . . . . . . . 12

2.3.1 Delaunay triangulation . . . . . . . . . . . . . . . . 13

2.3.1.1 Incremental algorithm . . . . . . . . . . . . . . . . 15

2.3.2 Constrained Delaunay triangulation . . . . . . . . . . . . . . . 19

2.3.3 TIN corrections . . . . . . . . . . . . . . . . . . . . 22

2.3.3.1 Flat areas . . . . . . . . . . . . . . . . . . . . 22

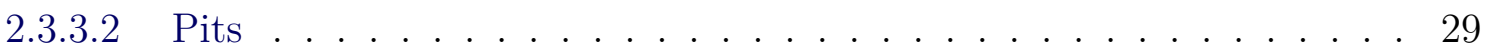

3 HYDROLOGICAL STRUCTURES . . . . . . . . . 35

3.1 Regular grid hydrological structures . . . . . . . . . . . . . . . . . 35

3.2 TIN hydrological structures . . . . . . . . . . . . . . . . . . . . 37

3.2.1 Gradient method . . . . . . . . . . . . . . . . . . 40

3.2.1.1 Drainage paths . . . . . . . . . . . . . . . . 42

3.2.1.2 Drainage graph . . . . . . . . . . . . . . . . . . 45

3.2 .2 Accumulated flows . . . . . . . . . . . . . . . . . . . . 48

3.2 .3 Drainage networks . . . . . . . . . . . . . . . . 55

3.2.4 Watersheds and subwatersheds delineation . . . . . . . . . . . . 59

4 RESULTS ............................. 63

4.1 Area of study . . . . . . . . . . . . . . . . . . . . 6 63

4.2 Reference drainage network . . . . . . . . . . . . . . . 64

4.3 TIN results . . . . . . . . . . . . . . . . . . 65 
4.3.1 Drainage paths . . . . . . . . . . . . . . . 65

4.3 .2 Drainage networks ...................... 66

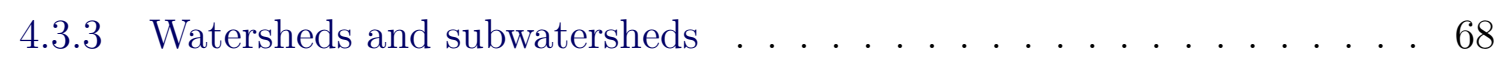

4.3.4 Computational times . . . . . . . . . . . . . . 70

4.4 TIN and regular grid results comparisons . . . . . . . . . . . . . 71

4.4 Drainage networks . . . . . . . . . . . . . . . 73

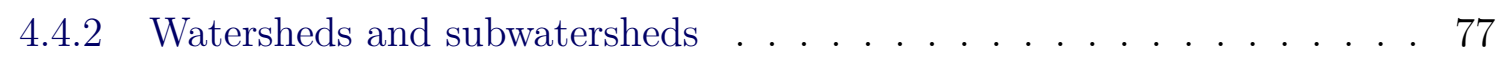

4.4 .3 Computational times . . . . . . . . . . . . . . . 82

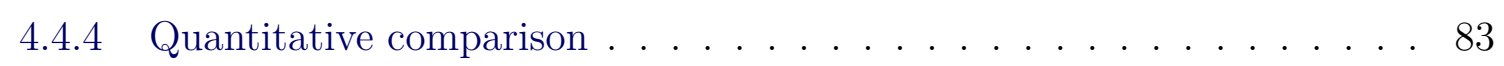

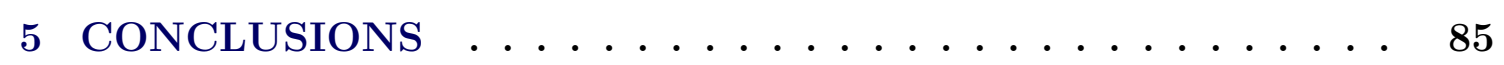

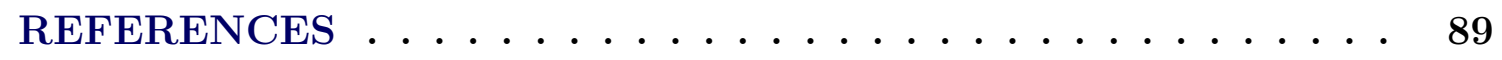




\section{INTRODUCTION}

Digital Elevation Models (DEMs) are important objects with relevant and useful applications in many areas of natural sciences. These models can be represented by different structures and the most common type of representation is by regular grids (FOWLER; LITTLE, 1979; PETRIE; KENNIE, 1987) as available from the Shuttle Radar Topography Mission (SRTM) (JARVIS et al., 2008), a well-known source of elevation data provided by the CGIAR-CSI.

In a regular grid, elevation values are defined in points or cells uniformly distributed over space as a two-dimensional matrix. Depending on the grid resolution, i.e., the number rows and columns, this structure may be well adjusted in regions with high variation in the elevation values although spatially redundant in regions where this elevation variation between cells is low or non-existent (in the case of flat areas). Usually, terrain surfaces are not regular in nature, so their representation is not required to be regular.

Another type of DEM representation is a Triangulated Irregular Network (TIN) (PETRIE; KENNIE, 1987; JONES et al., 1990; BERG et al., 2008), which is an efficient terrain model structure. In a TIN, the density of information can vary from region to region in a way that more points are included where there is more variation in the elevation values while fewer points are necessary in regions of less variation, thus avoiding data redundancy. The TIN model is comprised of several triangles generated from a set of points adequately distributed over space, where each point is defined by a triple $(x, y, z)$ with $x$ and $y$ as the coordinates on the horizontal plane and $z$ as the elevation value. These points ideally constitute the main characteristics and features of the terrain, so that a TIN can efficiently adapt to its irregularities.

Terrain surfaces represented by TINs are modeled with adjacent triangles of different sizes and shapes, where each triangle defines a linear plane in the portion of space that contains it. In addition, as there are many possible and distinct triangulations that can be calculated from the same set of points, it is necessary to generate a triangulation that results in a good approximation for terrain modeling, which is usually given by the Delaunay triangulation (JONES et al., 1990; BERG et al., 2008).

In this work, the Delaunay triangulation is calculated from an input dataset represented by contour lines and sampled points, where the contour lines comprise segments of successive points with same elevation value. As a consequence, it is likely that the triangulation may present edges crossing the contour line segments 
and these intersections could result in spurious terrain features. For this reason, intersections are removed by performing a further procedure that modifies the connections between points which define edges of the triangulation, so that each contour line segment is considered as a restriction line that cannot be crossed, resulting in a constrained Delaunay triangulation (ZHU; YAN, 2010).

Another problem that may occur in a TIN generated from contour lines is the existence of flat triangles. These triangles define flat areas where it is not possible to determine a flow direction because all three points or vertices of each triangle have the same elevation value, thus creating hydrological inconsistencies in the terrain model. This problem is solved by an improved procedure modified from a previous work (BARBALIC; OMERBEGOVIC, 1999), which inserts new points into the triangulation with interpolated elevation values. The procedure defines paths of flat triangles in the search for a point with a distinct elevation value used for the interpolation, then after a re-triangulation with the new points, each new triangle created has a defined flow direction and flows have no discontinuities.

Besides flat areas, an additional problem in terrain models is the presence of pits. In a TIN model, pits are points where no neighboring point connected by a triangle edge has a lower elevation value. Flows passing through these points do not follow any direction, hence determining discontinuities that must be avoided. The procedure used for pit removal defines paths of points that start from the pit and traverses triangles edges until reaching another point of lower elevation relative to the pit (SILVEIRA; OOSTRUM, 2007; SILVEIRA, 2009), then each point has its elevation value re-interpolated. This is a common solution to remove pits from regular grids known as carving process (SOILLE et al., 2003), but here performed on the TIN.

The generation of more exact and sophisticated terrain models brings many possibilities of use in applications of natural sciences since these models can be included and processed in computational systems known as GIS (Geographic Information System). For applications in hydrology, DEMs are used to generate flow directions, drainage networks, watersheds as well as many other hydrological structures.

The present work proposes a methodology for the automatic extraction of the aforementioned hydrological structures from a TIN terrain model based on the gradient method (JONES et al., 1990), which considers that each plane defined by a triangle determines the flow direction from its gradient vector. The methodology comprises procedures and algorithms that trace drainage paths by ordering and 
processing the triangles with the centroid's elevation as an associated priority and these paths are then connected to define a drainage graph structure, which allows to generate drainage networks from accumulated flows with the subsequent delineation of watersheds and subwatersheds.

One consideration to be made at this point is that, after the conclusion of this work, the author took knowledge of another work recently published which presents a different methodology for the extraction and generation of hydrological structures such as drainage networks, watersheds and subwatersheds from accumulated flows defined over digital elevation models represented by triangulated irregular networks (GUODONG et al., 2014). This other method also determines flow directions according to the gradient vectors, although it considers the flows only over triangles edges, either along channel edges or edges chosen from a particular condition that verifies the difference between two contributing areas in a triangle, which are separated by its gradient vector. This approach constitutes a simplified model that changes flow directions and inserts a restriction in the sequence of flows since it is defined only over the edges of the TIN.

The methods described here can be added as hydrology-specific functionalities in GIS applications capable of storing and processing a wide variety of georeferenced data. Furthermore, TIN datasets used for terrain modeling and analysis raise many challenges in the development of efficient algorithms to process and extract useful results from them because their use usually involves complex tasks. Many GIS applications that process terrain models have limited capabilities when it comes to flow modeling on TINs because it is required to design more robust data structures and algorithms to solve specific problems of computational geometry, so that this type of functionality is less developed than for the most common and simple DEM represented by regular grids.

All the procedures mentioned so far are performed as given by the sequence of steps summarized in figure 1.1. Each step represents a specific problem solved before the next one can be addressed. 

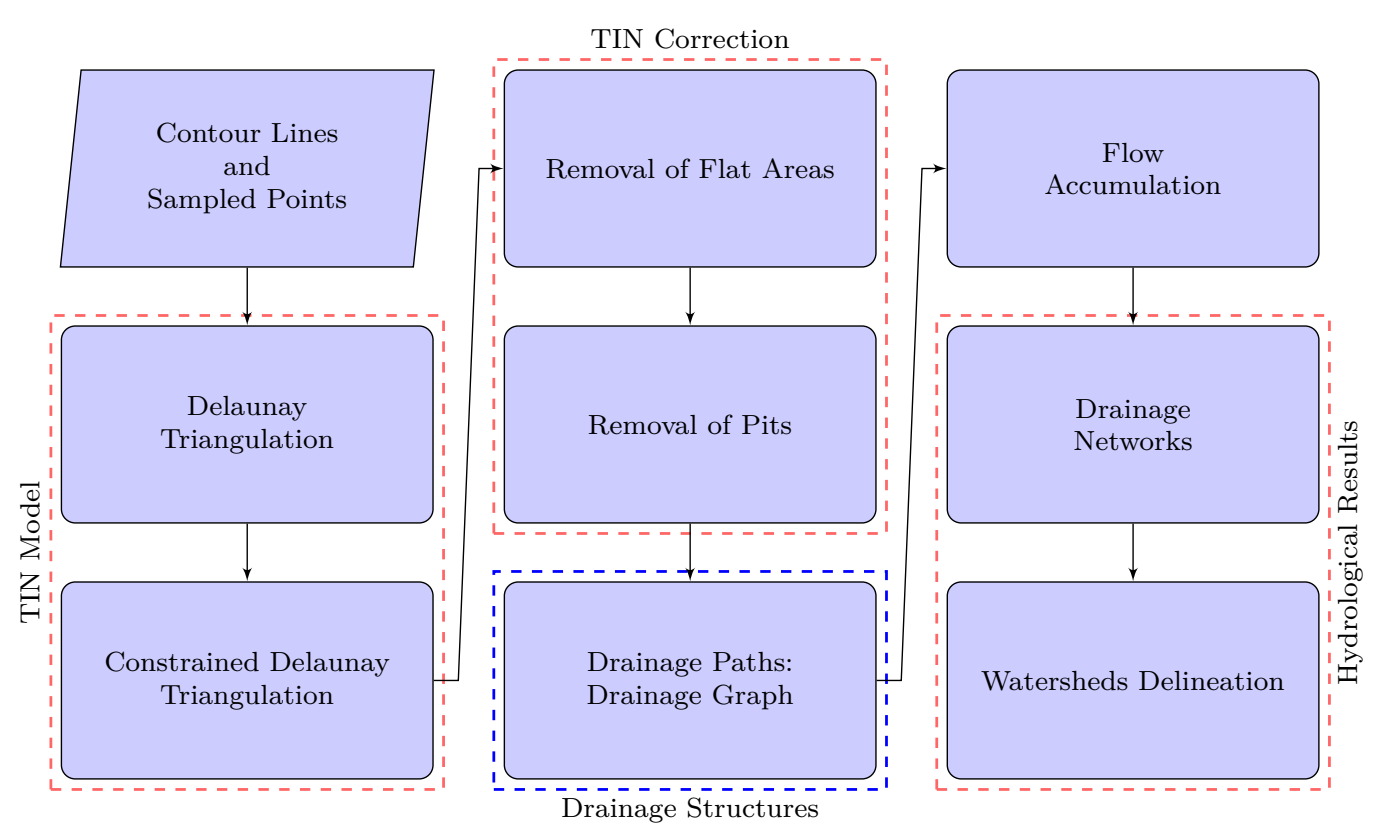

Figure 1.1 - Flowchart of the steps performed in this work

\section{$1.1 \quad$ Objectives}

\subsubsection{General objective}

The general objective of this work is the development of a new methodology for the extraction and generation of hydrological structures such as drainage networks and watersheds from terrain models represented by TINs. The methodology is based on the gradient method that produces drainage paths used to define a drainage graph structure that allows the calculation of accumulated flows over the TIN model.

\subsubsection{Specific objectives}

Specific objectives comprise solutions to common problems that usually occur when using DEMs as representations of terrain surfaces, in the case of this work, the use of TINs. Major problems are the existence of flat areas and the presence of pits.

Another specific objective is to perform comparisons and analyses between the hydrological results generated from the TIN and from the regular grid, so that this work considered a qualitative comparison. 
The specific objectives are as follows:

- Removal of flat areas: from the definition of paths of flat triangles while getting information of the elevation values from neighboring contour lines, then inserting new points into the triangulation with interpolated elevation values.

- Removal of pits: from the definition of paths of points starting from the pit and ending in points of lower elevation values, then re-interpolating the elevation values of all the points.

- Qualitative comparisons and analyses between hydrological results from the TIN and from the regular grid.

\subsection{Contributions}

The procedures developed for removing flat areas is an improvement on a previous work (BARBALIC; OMERBEGOVIC, 1999) since the existent method does not provide a solution to flat areas where the definition of a path of flat triangles does not include a point with a distinct elevation value. In this case, the solution proposed is to get information of the elevation values from neighboring contour lines while traversing the flat triangles, where the variation between the elevation values of these contour lines indicate whether the new points to be added into the triangulation should be interpolated either upwards or downwards.

The removal of pits is based on the definition of paths of points (SILVEIRA; OOSTRUM, 2007; SILVEIRA, 2009) with a subsequent re-interpolation of the elevation values of the points included in the path. This is a straightforward solution commonly used for pit removal from regular grids known as carving process (SOILLE et al., 2003), although in this work performed on the TIN considering whether the paths of points include contour line points or not.

The most important contribution of this work is a drainage graph structure defined from drainage paths. The drainage graph contains nodes created from the intersections between drainage paths segments and triangles edges. This structure allows the calculation of the flows accumulated in each node and, as a result, drainage networks are generated according to given threshold values. Additionally, watersheds and subwatersheds are delineated from the resulting drainage networks. 


\subsection{Work organization}

This work is organized as follows. Chapter 2 contains a detailed description of the Delaunay triangulation and the Incremental algorithm used for its calculation, as well as the procedure performed to generate the constrained Delaunay triangulation. It also describes the procedures and algorithms developed for removing flat areas and pits from the TIN model. Chapter 3 presents the methodology used for tracing drainage paths over the TIN based on the gradient method in order to define the drainage graph structure proposed in this work. Procedures of accumulated flows, drainage networks, watersheds and subwatersheds delineation are also presented. In chapter 4, all the hydrological results generated from the TIN such as drainage paths, drainage networks, watersheds and subwatersheds are qualitatively compared to the results generated from regular grids processed by the TerraHidro computational platform developed at INPE and also to an available drainage network produced by specialists and considered here as a reference of the main courses of water over the terrain. Computational times obtained from the algorithms are also given. Chapter 5 presents the conclusions as well as suggestions for future work. References are listed at the end. 


\section{DIGITAL ELEVATION MODELS}

Terrain surfaces can be digitally represented by elevation models which are useful in real and practical applications of natural sciences since these models support the modeling of natural phenomena in a computer. Specifically, hydrology applications usually use terrain models to automate the generation of drainage patterns and hydrological structures such as flow directions, drainage networks and watersheds.

Representations of terrain surfaces typically involve one of three distinct schemes (JONES et al., 1990), each one with its advantages and disadvantages: a rectangular grid of points; a set of points randomly scattered over the surface and connected to form a triangulation; or contour lines, as presented in topographic maps.

\section{$2.1 \quad$ Regular grids}

Regular grids are simple structures used for the representation of terrain surfaces. This structure is comprised of cells uniformly distributed over space and organized in rows and columns as a two-dimensional matrix, where each cell contains the information of the elevation value of a particular point in the surface. Figure 2.1 is a $3 \mathrm{D}$ visualization of the values stored in a regular grid. It is possible to notice the presence of a flat area located at its bottom part.

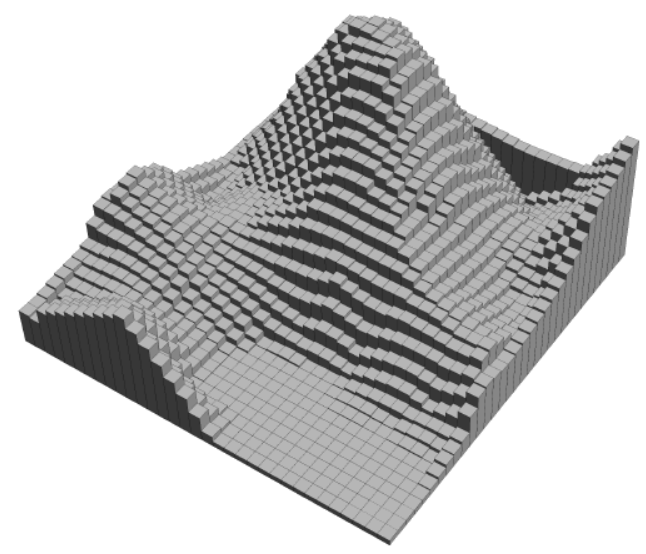

Figure 2.1 - 3D view of a regular grid

SOURCE: Tsirogiannis (2011)

In a regular grid, the adjacency between cells is explicitly defined. Considering a cell $(i, j)$ located at row and column indexed by $i$ and $j$, respectively, its eight neighbors are accessible with a simple increment or decrement of one unit on these indexes. 
Although regular grids present as main advantage a simple 2D-matrix structure with indexed cells, some drawbacks of using them as terrain models are:

- Each grid cell is assigned an elevation value generated from an interpolation process, so that much of the information from the original elevation data is lost in this process;

- The storage of highly redundant data in regions of smooth terrain (FOWLER; LITTLE, 1979), thus creating flat areas that can become larger depending on the grid resolution, making the extraction of drainage paths relatively difficult for any method, especially the ones based on local operations (O'CALLAGHAN; MARK, 1984)

- Flows with fixed directions assigned to each cell from only eight different possibilities, either two vertically, two horizontally or four diagonally, so restricting the flow directions which might generate unnatural drainage patterns.

Several methods and techniques are found in the literature for the extraction of hydrological structures from regular grids. Descriptions of detailed methods are given in O'Callaghan and Mark (1984) and Jenson and Domingue (1988), where the authors define the well-known D8 algorithm. This name is due to the flow direction of each cell be assigned to one of its eight neighbors, always following the direction of steepest descent.

The extraction of drainage networks from regular grids by using the methods mentioned above considers flows accumulated from cell to cell in paths formed by following the flow direction assigned to each cell. Drainage networks are then defined by given threshold values related either to the number of cells or the total area accumulated in the cells. Delineation of watersheds and subwatersheds from the drainage networks is performed by starting from an initial cell in the drainage network and then traversing all the cells reached from upstream flows, so that this process repeats for each cell reached while traversing the grid by following the upstream flows.

\subsection{Contour lines}

Contour maps are one of the first datasets developed to represent terrain elevations of the earth's surface, being an important source of digital elevation data 
(O'CALlAGHAN; MARK, 1984). Contour maps are defined by contour lines that can be digitized and then used to generate other DEM structures such as regular grids and TINs through specific methods.

In this work, contour lines and sampled points manually extracted from contour maps of the city of São José dos Campos (Brazil) were used as input data for the calculation of the triangulation that represents the terrain model. This dataset comprises a total of 202137 points irregularly distributed over the city's region, where neighboring contour lines present elevation differences of $5 \mathrm{~m}$ between one another. All the elevation data was processed as a sequence of points separated by dummy values, georeferenced in UTM coordinates and with the elevation values in meters. Successive points define contour lines and isolated points represent sampled points.

The entire dataset was taken from a database named 'Cidade Viva' (PREFEITURA MUNICIPAL DE SAO JOSE DOS CAMPOS, 2003), which is updated every 6 months and made publicly available since 2003 by the city's Geoprocessing Service of the Urban Planning Department in a format easily imported by a GIS. Figure 2.2 is an illustration of the contour lines and sampled points of the 'Cidade Viva' database for a small extent of the region considered in this work, georeferenced in UTM coordinates.

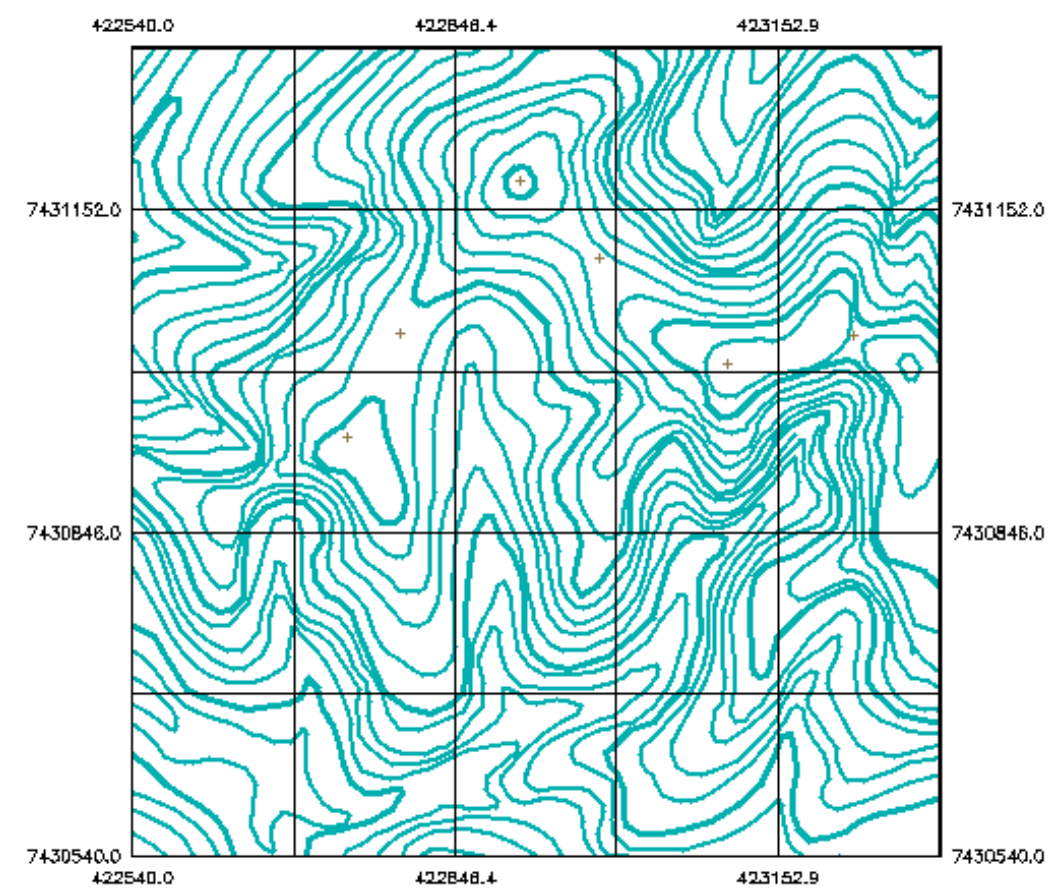

Figure 2.2 - Contour lines (green) and sampled points (cross-shaped symbols in brown) define elevation data 


\subsubsection{Input data corrections}

As the contour lines from the 'Cidade Viva' database were generated from contour maps by a manual process, these data were prone to the occurrence of errors which were easily detected since they created inconsistencies in the terrain model and, consequently, some problems were encountered when processing the flow directions and drainage networks in subsequent steps.

All the errors found in the input data were manually corrected after a visual inspection on the contour lines and sampled points, although it is of interest to devise algorithms that can automatically correct each error as it constitutes a timeconsuming task. An automatic procedure to solve these specific errors is not a trivial problem to be tackled since it involves complex computational geometry algorithms which are beyond the scope of this work.

The main problems that occurred when processing the erroneous input data, which then allowed the detection of errors, are listed as follows:

- The procedure devised to remove flat areas interpolated incorrect elevation values to the new points inserted into the triangulation because the interpolation process depends on a path of flat triangles traversed inside a contour line and, with the presence of flawed contour lines, the path followed a wrong way and the new elevation values were miscalculated;

- Pits were created at points with incorrect interpolated elevation values after the removal of flat areas and also from inconsistent elevation values of sampled points;

- Drainage paths presented flow discontinuities, which generated a disconnected drainage network and, consequently, an incorrect delineation of watersheds.

Some examples of the inconsistencies found in the input data can be visualized in figure 2.3, where specific problems are highlighted by a red rectangle. The main existing problems depicted in the figure consist of broken contour lines, contour lines crossing each other and inconsistent elevation values of contour lines and sampled points. 


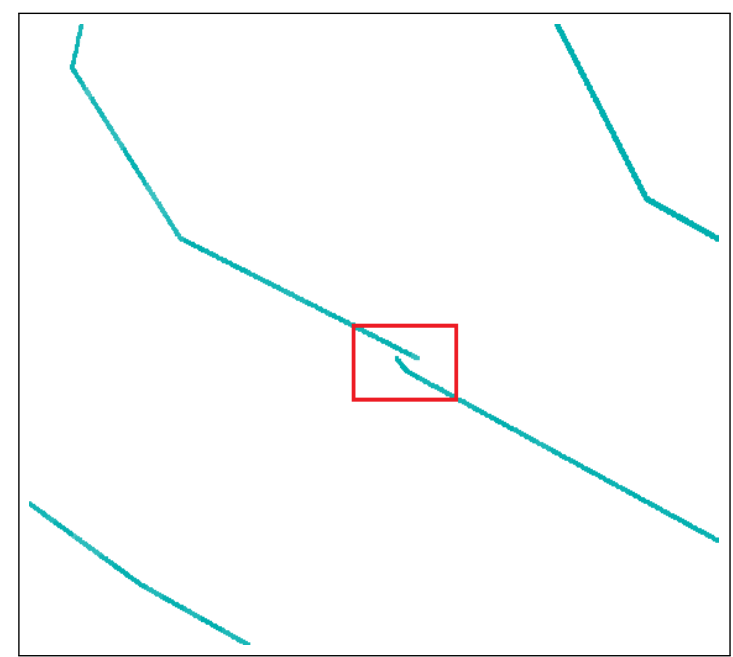

(a)

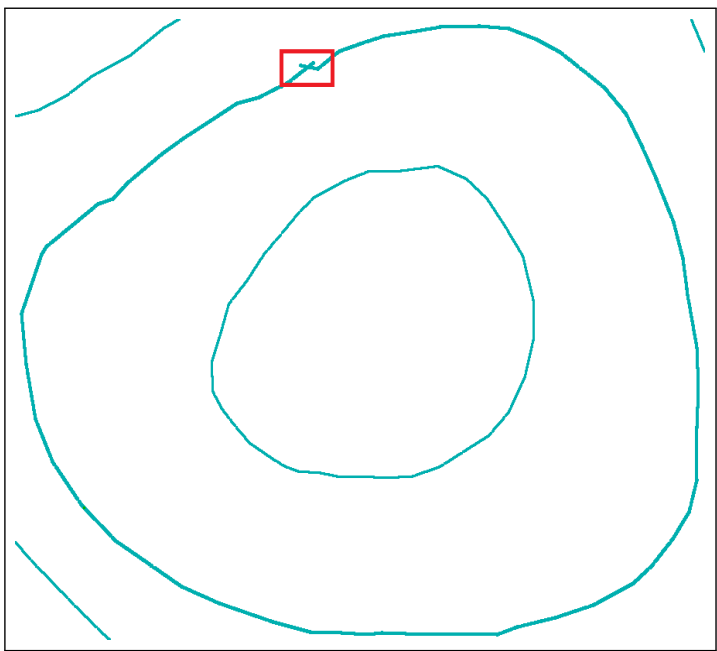

(c)

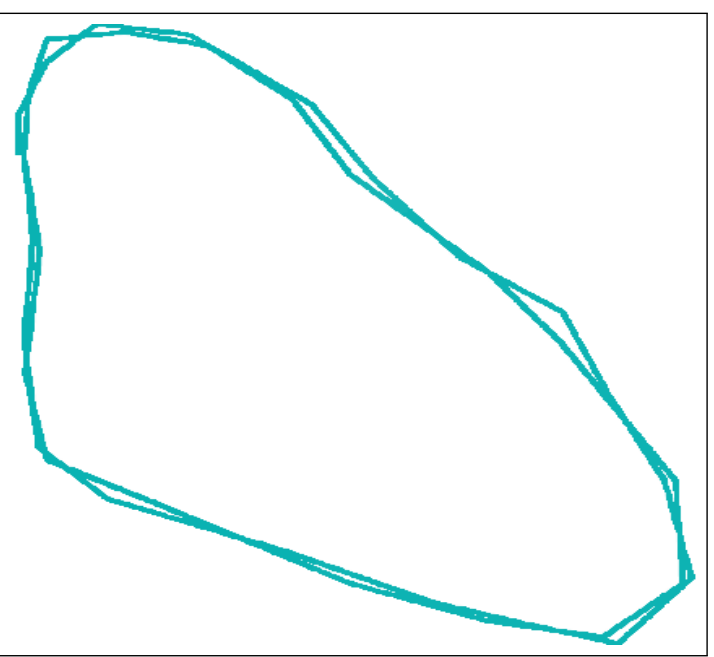

(b)

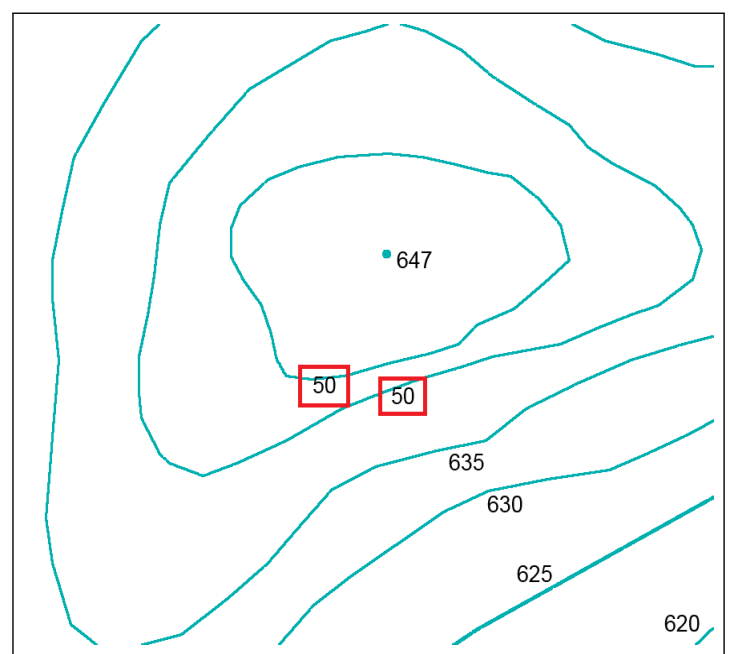

(d)

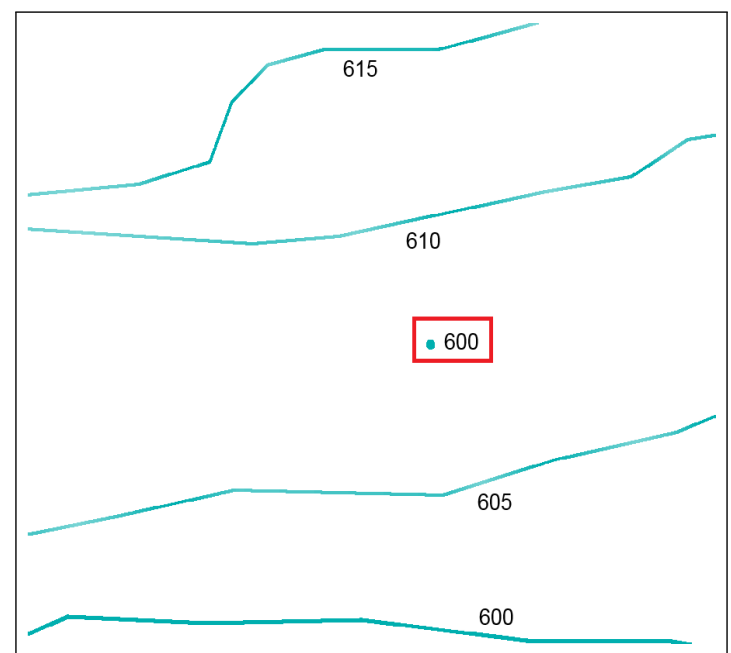

(e)

Figure 2.3 - Input data inconsistencies: (a) broken contour line. (b) contour lines crossing each other. (c) contour line is not closed. (d) inconsistent elevations of contour lines. (e) inconsistent elevation of sampled point. 
As an example of how the input data was corrected, table 2.1 lists the sequence of points that comprises the broken contour line shown in figure 2.3(a). The left column contains each point from both parts of the contour line, which are separated before the correction. The right column presents the data of the contour line after the two parts have been connected together with the exclusion of some points. The excluded points are highlighted by red rectangles and indicate points that were removed since they do not define a smooth contour line.

Table 2.1 - Original and corrected sequences of contour line data

\begin{tabular}{|c|c|}
\hline Original contour lines & Corrected contour line \\
\hline$y$ & $z$ \\
\hline & \\
\hline 419440.9040477432511 .254837670 & 419443.4307377432521 .996772670 \\
\hline 419447.2207737432502 .408537670 & 419440.9040477432511 .254837670 \\
\hline 419461.1175727432496 .089751670 & 419447.2207737432502 .408537670 \\
\hline$-1-1-1$ & 419460.4858997432495 .457873670 \\
\hline 419459.8542267432496 .089751670 & 419483.8577887432484 .084059670 \\
\hline 419460.4858997432495 .457873670 & 419524.2848387432461 .336431670 \\
\hline 419483.8577887432484 .084059670 & 419541.9716727432448 .698860670 \\
\hline$\ldots$ & $\ldots$ \\
\hline
\end{tabular}

Additionally, inconsistent elevation values of contour lines and sampled points were removed by modifying the values according to the surrounding terrain information in order to guarantee a better quality terrain model. For instance, the incorrect elevation values of the contour lines presented in figure 2.3(d) were modified from the value 50 to the values 640 and 645 , while the elevation value from the sampled point in figure 2.3(e) was modified from 600 to 607.

\subsection{Triangulated irregular networks}

The set of triangles from a Triangulated Irregular Network (TIN) defines a good approximation to the irregularities inherent to a terrain surface since the TIN structure works as a terrain model calculated from surface-specific points that represent the main features of the terrain, which are also considered as the backbone of the surface (FOWLER; LITTLE, 1979).

Triangle-based models provide a more accurate representation of the original data (JONES et al., 1990) as the elevation values associated to each point are not modified, although the triangles define linear planes where new points can be interpolated. As 
previously mentioned, the present work considers the TIN defined by a constrained Delaunay triangulation which is generated from a set of points that defines contour lines and sampled points. Figure 2.4 exhibits a 3D view of a TIN.

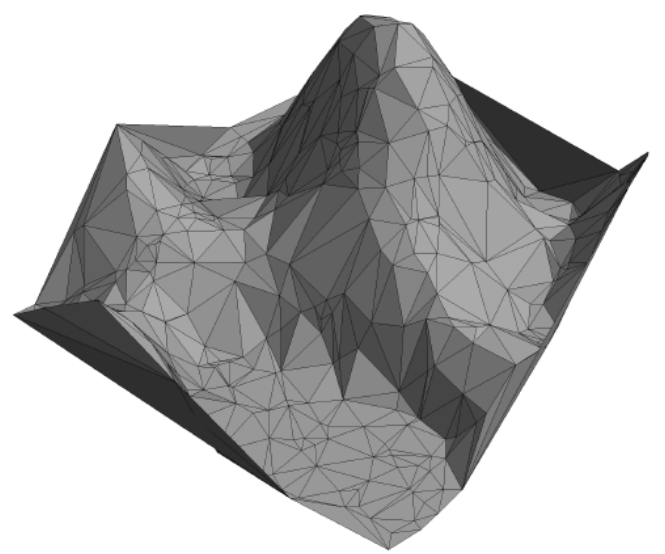

Figure $2.4-3 \mathrm{D}$ view of a TIN

SOURCE: Tsirogiannis (2011)

\subsubsection{Delaunay triangulation}

According to Berg et al. (2008), the Delaunay triangulation produces good approximations and defines a more suitable triangulation for terrain modeling because it avoids the generation of skinny triangles. These triangles may create terrain inconsistencies such as the one shown in figure 2.5.

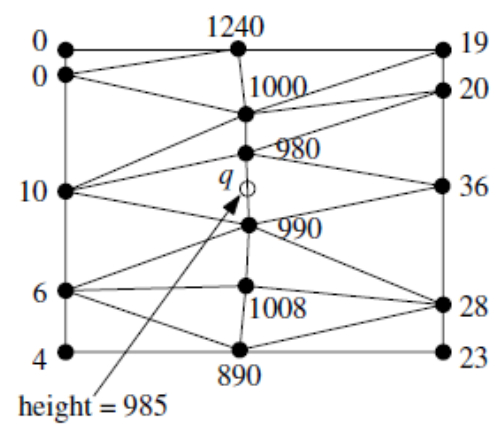

(a)

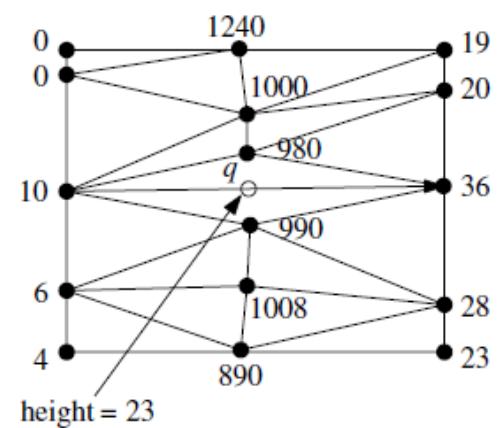

(b)

Figure 2.5 - Connections of triangles edges may create terrain inconsistencies: (a) correct interpolated elevation. (b) incorrect interpolated elevation. SOURCE: Berg et al. (2008) 
In this example, the right triangulation contains an edge connecting points that would result in a hole in the middle of a mountain ridge, as the point $q$ would have an elevation value interpolated between two lower points, while the elevation values of the middle points are higher in relation to those at the sides. The left triangulation does not present this problem as the 'incorrect' edge is flipped.

The main property of the Delaunay triangulation is that each triangle defines a circle through its three vertices that does not contain any other point of the set in its interior. This property is considered as a criteria for calculating the triangulation (TSAI, 1993), which indicates that a Delaunay triangulation consists of more equiangular triangles. Consequently, the minimum angle among all the triangles is maximized. Figure 2.6 shows a Delaunay triangulation together with the Delaunay criteria for a circle defined by the three vertices of a triangle.

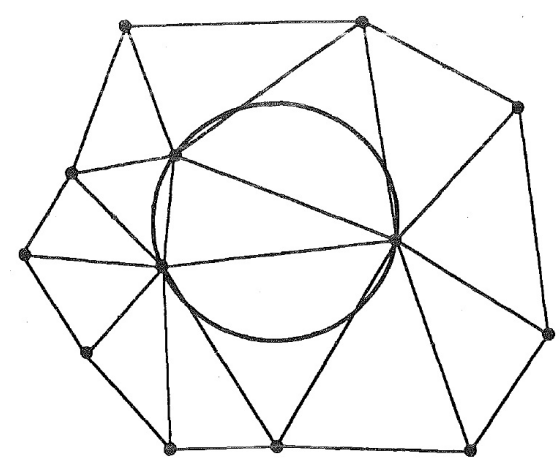

Figure 2.6 - Delaunay triangulation criteria SOURCE: adapted from Jones et al. (1990)

The Delaunay triangulation calculated from a set of points on the plane can be generated by several different algorithms found in the literature, each one with a specific approach. Some of these algorithms are: Bowyer-Watson (BOWYER, 1981; WATSON, 1981), Incremental (GUIBAS et al., 1992; BERG et al., 2008), Divideand-Conquer (CIGNONI et al., 1998), Fortune (FORTUNE, 1987) and Brute Force (O'ROURKE, 1998). In the present work, the Incremental algorithm was used because its time complexity is $O(n \log n)$ (BERG et al., 2008), which is an upper bound for the running time of the algorithm with an input dataset of size $n$.

It is worthy mentioning that an implementation of the Incremental algorithm was developed because the structures and procedures from the source code could be adapted to the solution of the problems considered in this work, such as the removal 
of flat areas and pits as well as the generation of drainage paths. Specific information is stored in the structures in order to improve the performance of the algorithms and, consequently, their computational times. Another consideration is that these structures and procedures were implemented in $\mathbf{C}++$ and could be easily modified to work with the Terralib library (CAMARA et al., 2000) developed at INPE, a library of GIS classes and functions for spatial databases available as an open source project.

\subsubsection{Incremental algorithm}

The first step of the Incremental algorithm is to define a triangle that contains the whole set of points to be triangulated located in its interior. In addition, each one of its vertices cannot be inside the circle that passes through any three points of the set, thus respecting the Delaunay criteria.

The coordinates of the vertices of this initial triangle are defined by a constant value $\mathrm{K}$ calculated from a point $\mathrm{P}$ that defines the width and height of an enclosing rectangle of the set of points as presented by Vomacka (2008) apud Zalik and Kolingerova (2003), where these authors consider the value of $\mathrm{K}$ as ten times greater than the larger rectangle dimension, either width or height.

This work considers the value of $\mathrm{K}$ calculated from a straight line with a predefined negative slope, where this line is translated up and to the right in relation to the point $\mathrm{P}$, so that the intersections between the line and both the $x$ and $y$ axes determine the points $(K, 0)$ and $(0, K)$, respectively. The initial enclosing triangle is then defined by the vertices $(K, 0),(0, K)$ and $(-K,-K)$ as illustrated in figure 2.7 , where the black rectangle represents the enclosing rectangle of the set of points.

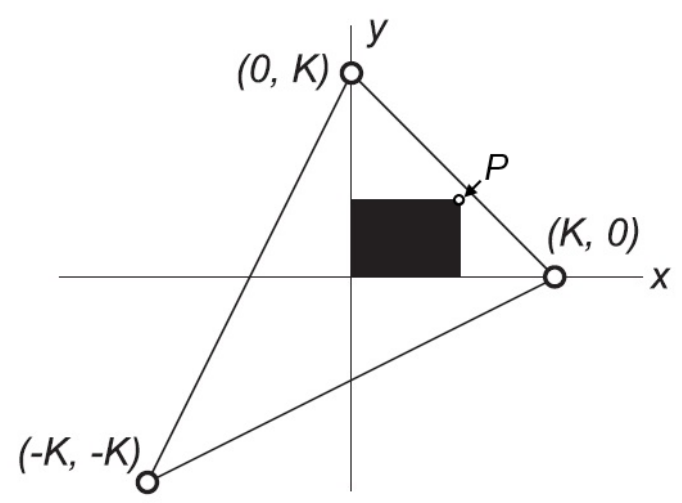

Figure 2.7 - Enclosing triangle of the set of points SOURCE: adapted from Vomacka (2008) 
After defining the initial triangle, each point of the set is inserted one at a time into the current triangulation, then new edges are created and connected to the inserted point. These new edges are either connected to each one of the three vertices of the triangle that contains the point, if the point is located inside the triangle, or to the vertices opposite to an edge shared by two triangles, if the point is exactly on an edge.

Both cases are illustrated in figure 2.8, where new edges between the inserted point and triangles vertices are depicted by dashed lines.

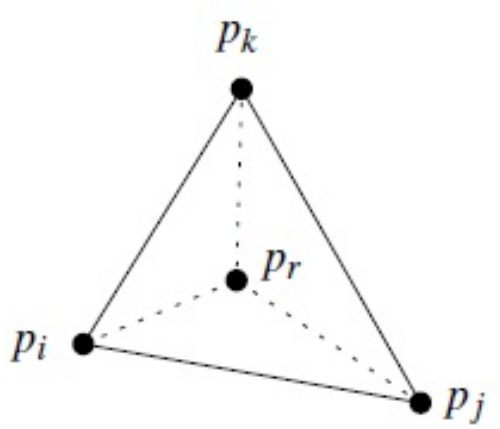

(a)

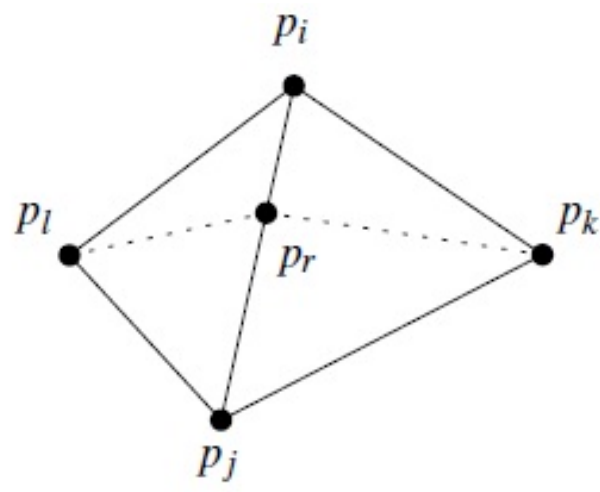

(b)

Figure 2.8 - Insertion of a point into the triangulation: (a) inserted point $p_{r}$ is located inside a triangle. (b) inserted point $p_{r}$ is on an edge.

SOURCE: Berg et al. (2008)

The new edges of the triangles created after the insertion of a new point are then considered as Delaunay edges, i.e., these edges respect the Delaunay criteria since the circle that passes through them does not include any other point in its interior.

However, the edges of each divided triangle need to be checked whether they are still Delaunay edges as a circle passing through both the points of one of the edges and also through the inserted point may include another point of the set inside it. In this case, the edge will be considered illegal. Figure 2.9 exhibits an example of illegal edge, where the circle that passes through the points of the edge $p_{i} p_{j}$ and also through the point $p_{k}$ includes the point $p_{l}$ in its interior.

Furthermore, when a triangle edge is considered illegal, it is modified by a rotation operation and its new points are the points opposite to the edge before the rotation, 


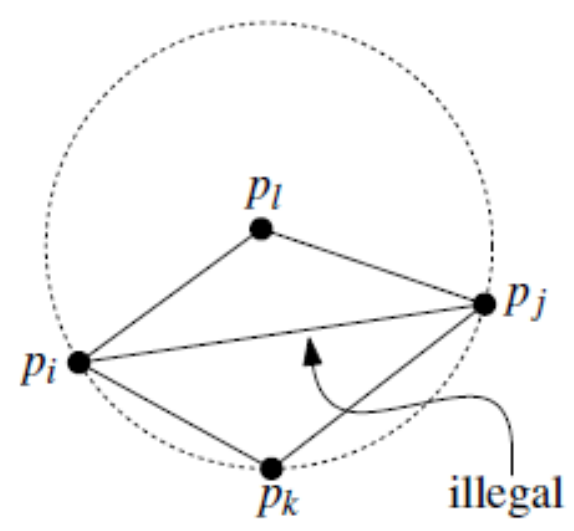

Figure 2.9 - Edge $p_{i} p_{j}$ is illegal

SOURCE: Berg et al. (2008)

so that both the triangles that shared the edge before it is rotated are also changed into two new triangles. The rotated edge becomes a Delaunay edge since the interior angles of the new triangles is maximized as shown in figure 2.10, where two skinny triangles are changed after the rotation of the edge $p_{i} p_{j}$ to $p_{k} p_{l}$.
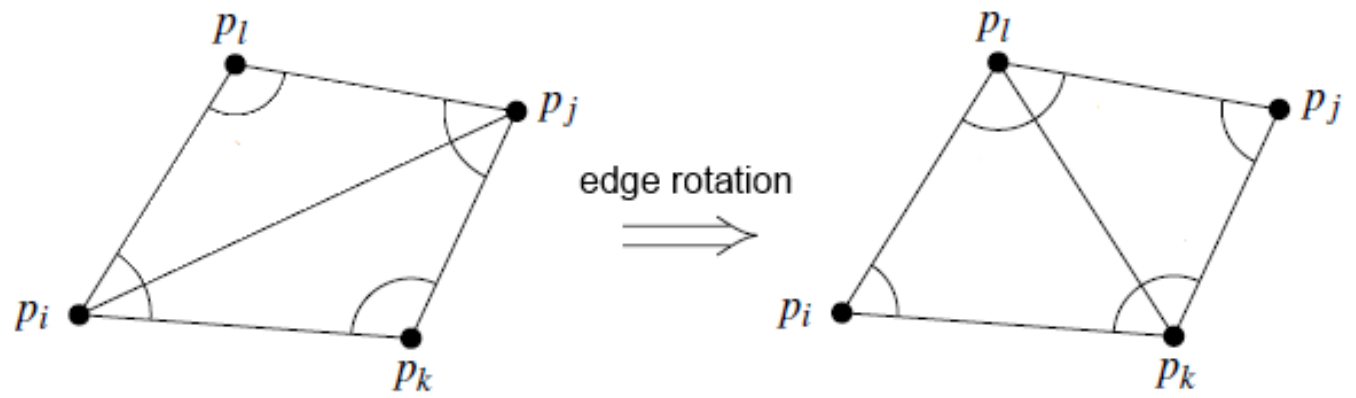

Figure 2.10 - Rotating an edge maximizes interior angles of triangles SOURCE: adapted from Berg et al. (2008)

It is important to notice that an edge rotation can make other edges become illegal, more precisely, the other two edges of the triangles that existed before the rotation. These edges also need to be checked if they are still Delaunay edges, otherwise they are rotated as well. So, after each edge rotation, this procedure of checking and rotating recursively repeats until no more edges are considered illegal. Therefore the triangulation is locally changed and triangles are modified around the inserted point. Figure 2.11 illustrates local changes made to a triangulation after a new point is inserted, where the region in gray represents all the triangles that were modified. 

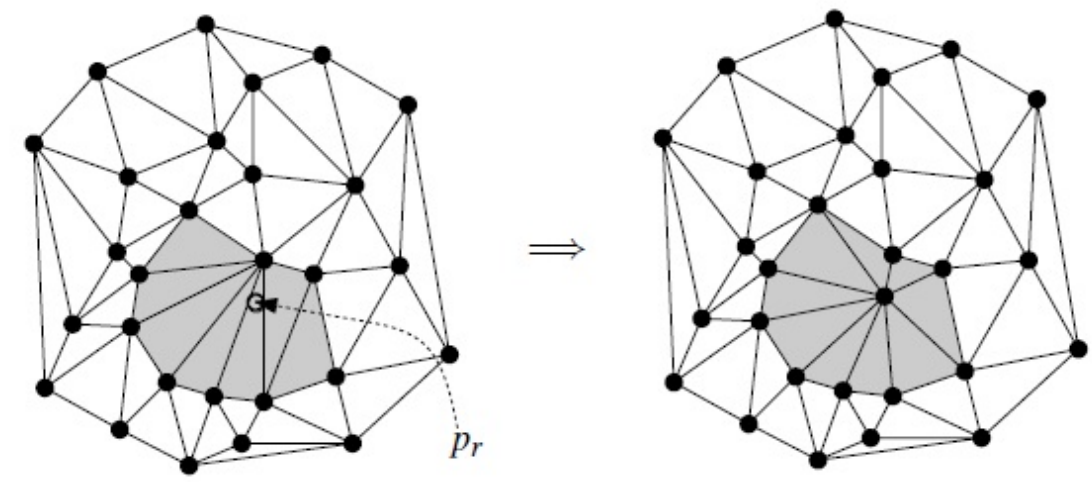

Figure 2.11 - A new point $p_{r}$ locally changes the triangulation SOURCE: Berg et al. (2008)

In the programming level, for the previously mentioned modifications to be correctly applied to the triangulation, the triangle structure holds the information of each adjacent triangle as a pointer reference and these references change with each edge rotation in order to ensure that the adjacency relationships are maintained.

Another key aspect of the Incremental algorithm is the usage of a tree structure for the storage of the triangles, which is mainly related to its $O(n \log n)$ time complexity. This structure stores each triangle as one of its nodes, where divided triangles and new triangles created are then connected in the tree by hierarchical links that indicate the triangles divisions. The new triangles are stored as children of the divided triangles, so that each triangle has two or three children depending on whether the inserted point is located inside a triangle or on an edge, respectively.

Basically, each inserted point determines new nodes in the tree and also new branches in a hierarchy between old and new triangles. At the end, Delaunay triangles are leaf nodes of the tree and the initial triangle, together with all its incident edges, is discarded. Thus the tree structure works as a history that reflects how the triangulation changes with each point insertion. An example of how the triangles and the tree structure are modified after a point is inserted can be visualized in figure 2.12 .

According to Berg et al. (2008), the total number of triangles created by the Incremental algorithm is linear and at most $9 n+1$, with $n$ the number of points to be triangulated. As the tree height is proportional to $\log (n)$, the search for a triangle that includes a specific point is performed in logarithmic time, resulting in the algorithm's time complexity of $O(n \log n)$. 

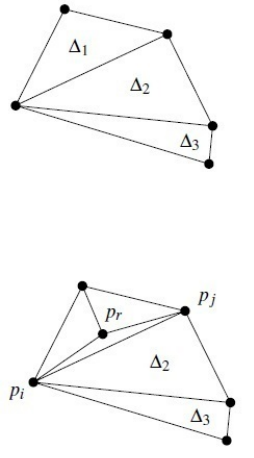

$\Delta \Delta_{1} \Delta_{3}$
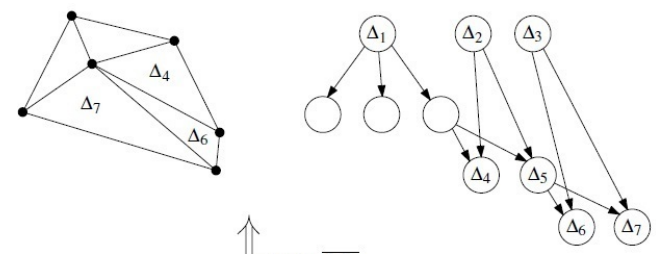

(42) $\Delta_{3}$

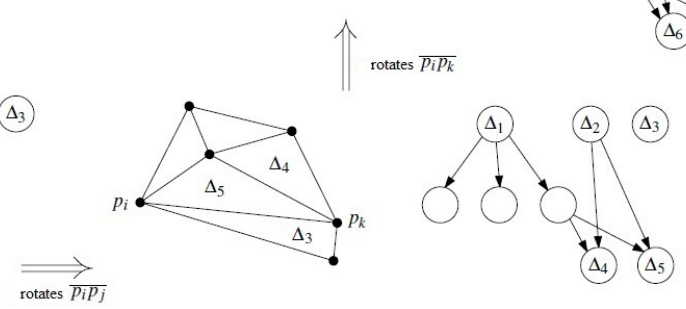

Figure 2.12 - Tree structure reflects triangles divisions SOURCE: adapted from Berg et al. (2008)

\subsubsection{Constrained Delaunay triangulation}

When the points used as input for calculating the triangulation do not have any specific connection, the Delaunay triangulation suffices to define a TIN as terrain model. However, as mentioned before, the set of points used in this work comprise contour lines and sampled points, so that each segment of a contour line is considered as a restriction line that cannot be crossed by a triangle edge (EASTMAN, 2001), otherwise the intersections may create inconsistencies in the terrain model.

In order to solve this problem, an initial Delaunay triangulation is calculated and a further procedure performs edge rotations removing the intersections between contour line segments and triangles edges (ZHU; YAN, 2010). Figure 2.13 exhibits triangles edges (dashed lines) that intersect contour line segments (solid lines) and the resulting triangulation after removing these intersections.
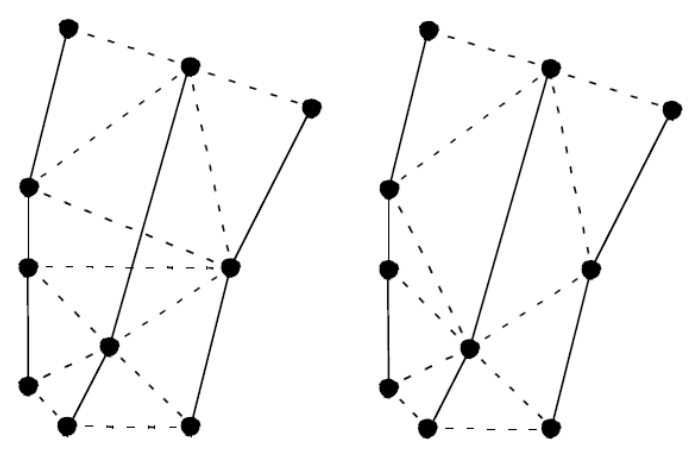

Figure 2.13 - Triangulations before and after removing intersections SOURCE: Eastman (2001) 
The procedure for removing intersections primarily detects, for each contour line segment, an initial triangle connected to one segment endpoint such that its edge opposite to the endpoint also intersects the segment. This initial triangle is added into a queue structure and all the adjacent triangles are checked if one of their edges intersects the segment as well. Thus each triangle that intersects the segment is then added into the queue and this verification process continues until no more intersections are found, i.e., when the other segment endpoint is reached.

All the triangles stored in the queue are then processed in pairs until all the intersections are removed. If an edge of a triangle intersects the segment, not in an endpoint, then its adjacent triangle also intersects the segment, so both triangles are removed from the queue, their common edge is rotated and the new triangles are then inserted back into the queue for a further verification.

This procedure continues as long as there are intersections between the contour line segment and triangles edges. In the end, the total number of triangles in the triangulation is not changed since an edge rotation between two triangles still defines two triangles. Figure 2.14 illustrates how intersections are removed at each step of the procedure. The red dashed line represents a segment with endpoints $A$ and $G$ that cannot be crossed by triangles edges. The queue state after each edge rotation is also shown. In this example, the following steps are performed:

a) Triangles are numbered from 1 to 5 with vertices referenced by the letters from $A$ to $G$, and the queue $Q$ is initially empty;

b) After performing the search process as described above, each triangle is inserted into $Q$;

c) Next, the head of $Q$ is triangle 1 and its adjacent triangle is 2 , so they are both removed from $Q$, their common edge is rotated and they are pushed back at the end of $Q$. The same happens to triangles 3 and 4, as triangle 3 is now in the head of $Q$;

d) Triangle 5 is the new head of $Q$ and its adjacent triangle is 4 , so an edge rotation is performed and they are pushed back at the end of $Q$;

e) The next head of $Q$ is triangle 1 and its adjacent triangle is 2 , but their common edge does not intersect the segment, so no edge rotation is performed and they are both removed from $Q$. This also happens to triangles 3 and 5, and the only triangle left in $Q$ is triangle 4; 
f) Finally, as the new head of $Q$, triangle 4 and its adjacent triangle, now triangle 2, have their common edge rotated and they are pushed back at the end of $Q$. After this last step, all intersections between triangles edges and the segment were removed and the procedure is finished.

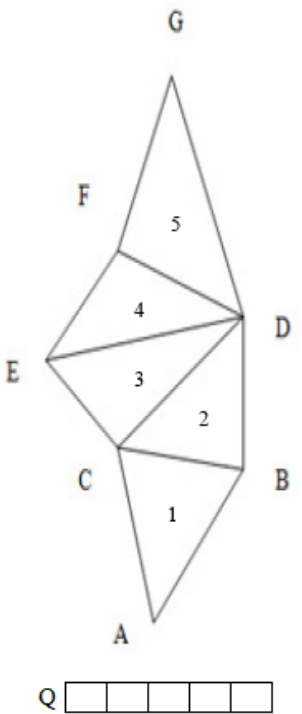

(a)

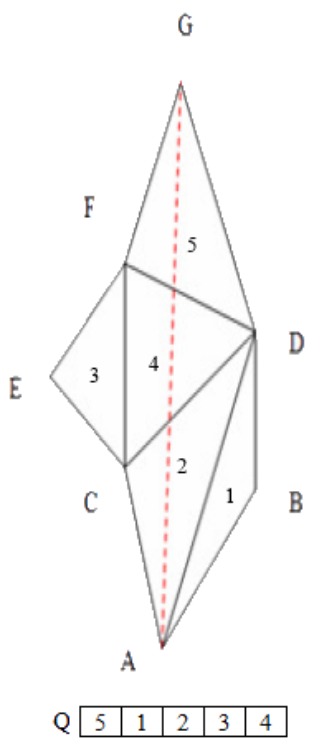

(d)

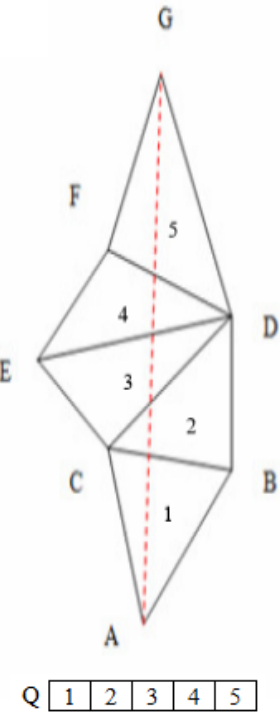

(b)

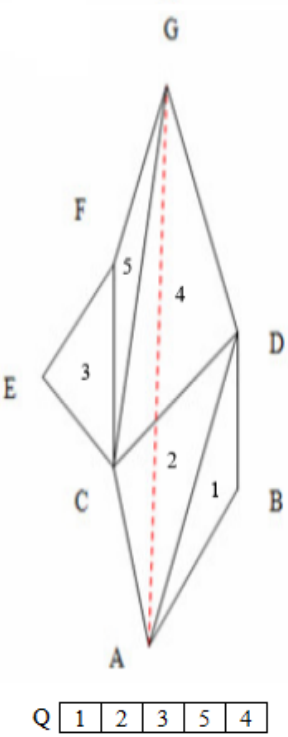

(e)

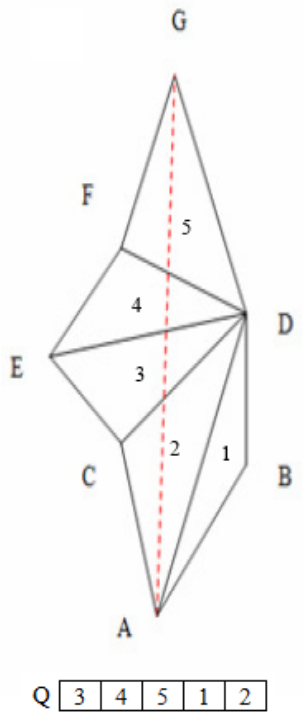

(c)

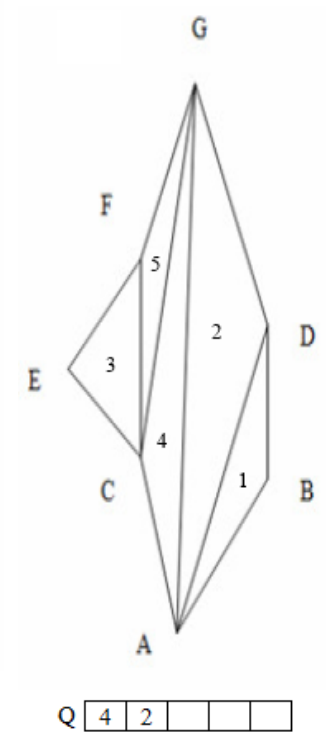

(f)

Figure 2.14 - Edges are rotated and intersections are removed SOURCE: adapted from Zhu and Yan (2010) 


\subsubsection{TIN corrections}

As already mentioned, DEMs are approximations of terrain surfaces that may present different types of problems. The most common problems are the presence of flat areas and pits, which produce hydrological inconsistencies since flow directions are not defined where they occur, thus generating discontinuities of flow. In order to solve these problems, some procedures were devised based on the definition of paths that include information from triangles and points, and this information is used to identify and correct both problems.

\subsubsection{Flat areas}

Flat areas usually occur over terrain surfaces in regions where slope variations are relatively low when compared to other regions that present more significant changes in elevation. For this reason, terrain models are prone to such inconsistencies as they are near-to-real approximations.

TINs used as terrain models present flat areas whenever the three vertices of a triangle have the same elevation value, thus defining a flat triangle. Since this work uses contour lines as input data, the resulting triangulation is likely to contain flat triangles as shown in figure 2.15. This situation must be avoided because flow directions are undefined over a flat area, which prevents continuity of flows and turns out to be a major problem in hydrological computations.

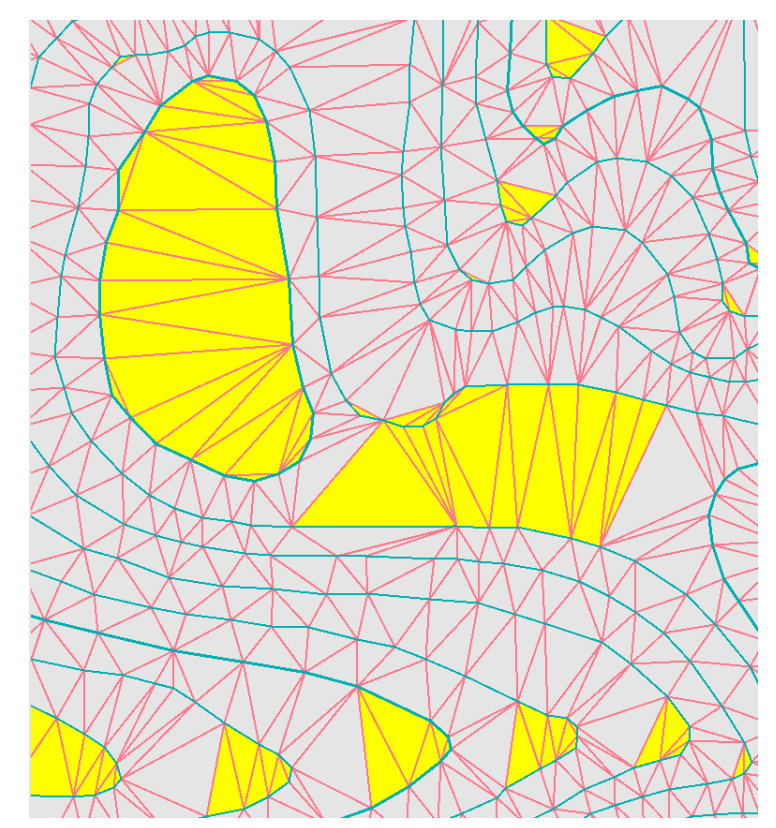

Figure 2.15 - TIN from contour lines with flat triangles (yellow) 
In this work, flat triangles are removed by the insertion of new points into the triangulation, where each new point has an interpolated elevation value. These points are considered as critical points placed at the middle of critical edges which are edges identified by two different cases:

a) An edge that connects two non-consecutive points on the same contour line;

b) An edge connecting two points of different contour lines but with equal elevation values.

Both cases of critical edges are illustrated in figure 2.16, where solid lines represent contour lines and dashed lines are triangles edges, with the critical edges in red.

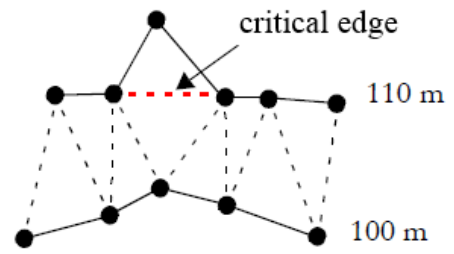

(a)

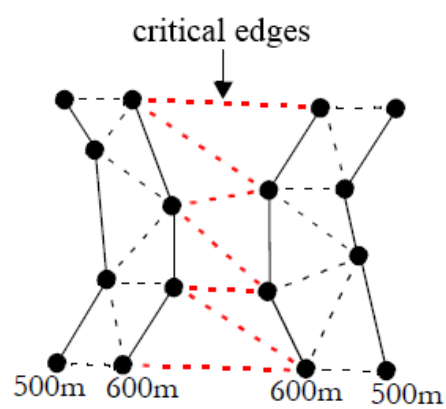

(b)

Figure 2.16 - Flat triangles and critical edges SOURCE: adapted from Eastman (2001)

After determining the critical edges, the next step is to select all the corner triangles, which are triangles that contain only one critical edge and two edges that are noncritical, i.e., either contour line segments or edges connecting points of different elevations. The corner triangles are considered as initial triangles for the definition of a path that includes the critical points where the elevation values are interpolated (BARBALIC; OMERBEGOVIC, 1999).

The path of triangles is then defined by a depth-first search procedure, starting at a corner triangle and always going from the current one to some of its adjacent triangles that shares a common critical edge and is not visited yet. Thus each triangle traversed in the search process determines a new critical point to be included for interpolation. 
At the beginning of the procedure, the point that connects the two non-critical edges of the corner triangle is defined as the first point in a sequence of points included for interpolation. Then whenever a critical edge leads to an unvisited triangle, its critical point is also added as a new point in the sequence and this procedure continues as long as there are unvisited triangles to be followed through the critical edges.

The search process terminates when it reaches a corner triangle that contains a point of different elevation value relative to the elevation value of the flat area. In this case, the point opposite to the critical edge of the last triangle is then added into the sequence as its final point. At the end, all the critical points included in the sequence have their elevation values linearly interpolated between the elevation values of the initial and final points.

This linear interpolation of elevation values considers the length of the segments inside a path as the distance from each critical point to the initial point (equations 2.1 and 2.2), which in turn is used as a weight factor for the interpolation of a critical point $c p_{m}$ (equation 2.3) (FREITAS et al., 2013).

Each value lengt $_{k}$ represents the distance between the critical points indexed by $k$ and $k-1$. The point $c p_{0}$ denotes the initial point and the point $c p_{n}$ the final point, in a path with a total of $n+1$ points. In addition, the values $i p$ and $f p$ in equation 2.3 are the elevations of the initial and final points, i.e., $c p_{0}$ and $c p_{n}$, respectively. The value $d i s t_{m}$ is the distance from the critical point $c p_{m}$ to the initial point and totaldist is the distance between the initial and final points, so that both distances are calculated as the sum of the lengths of each segment traversed in the path.

$$
\begin{gathered}
\text { dist }_{m}=\sum_{k=1}^{m} \text { length }_{k} \\
\text { totaldist }=\sum_{k=1}^{n} \text { lengt }_{k} \\
c p_{m}=\frac{\text { dist }_{m}}{\text { totaldist }}(f p-i p)+i p
\end{gathered}
$$

Figure 2.17 exhibits the critical points located on critical edges in dark blue, the corner triangles in blue and the paths of flat triangles in green, for the same region shown in figure 2.15. Initial and final points of the paths are in red. 


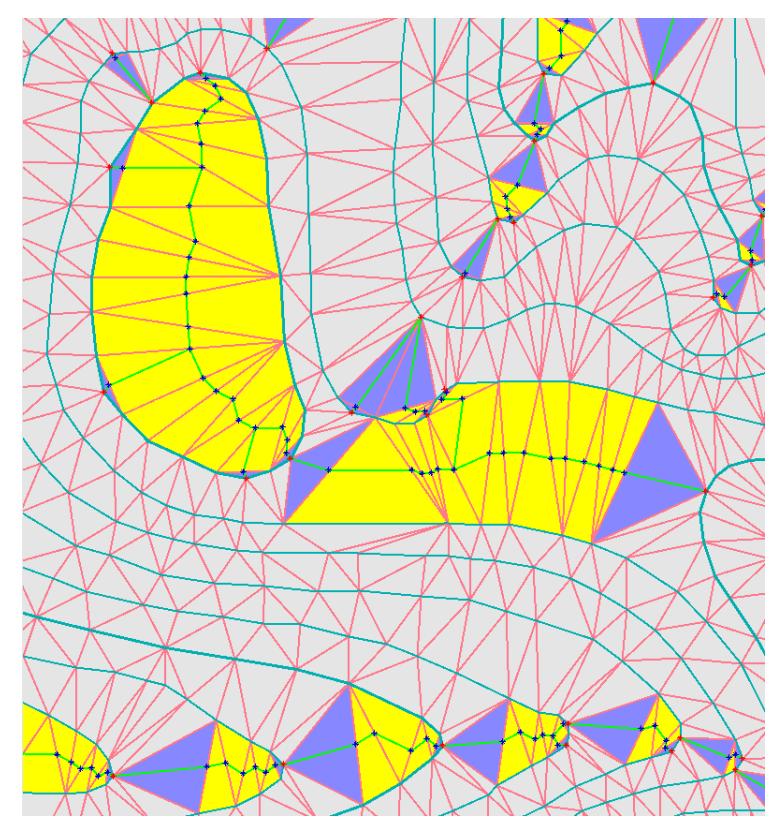

Figure 2.17 - Paths of triangles initiated at corner triangles define critical points for interpolation

An example of interpolated elevation values assigned to the critical points of a path are shown in table 2.2. The points highlighted by red rectangles are the initial and final points of the path, which remain unchanged in the interpolation process. Both the elevation values and distances are given in meters rounded to 2 decimal places.

Table 2.2 - Interpolated elevation values of critical points

\begin{tabular}{ccc}
\hline \hline \multicolumn{2}{c}{ Interpolated elevation values $z$} & Distance to the initial point \\
\hline$x$ & $z$ & \\
\hline 425491.8970807432509 .398924700 .00 & 0.00 \\
425494.6832817432508 .005368700 .43 & 3.11 \\
425496.7729327432505 .566648700 .87 & 6.32 \\
425500.6039597432507 .308591701 .45 & 10.53 \\
425504.0867117432507 .656980701 .93 & 14.03 \\
425508.2660137432506 .611814702 .53 & 18.34 \\
425511.0522147432503 .476315703 .11 & 22.53 \\
425515.2315167432500 .689206703 .80 & 27.56 \\
425519.0625437432499 .644040704 .35 & 31.53 \\
425523.1632517432497 .400159705 .00 & 36.20 \\
\hline \hline
\end{tabular}

Branches found while traversing triangles with three critical edges lead to more than one path, which are also processed after the interpolation procedure has assigned 
an elevation value to each critical point included in the first path. The search process repeats starting at each branching triangle, although the initial point used for interpolation is now one of the critical points of the branching triangle where the elevation value is already interpolated (FREITAS et al., 2013).

The same procedure is performed until all the critical points have been assigned an interpolated elevation value. Finally, these critical points are inserted into the triangulation and the areas around them are then re-triangulated. Figure 2.18 illustrates how branches are processed for different sequences of critical points.

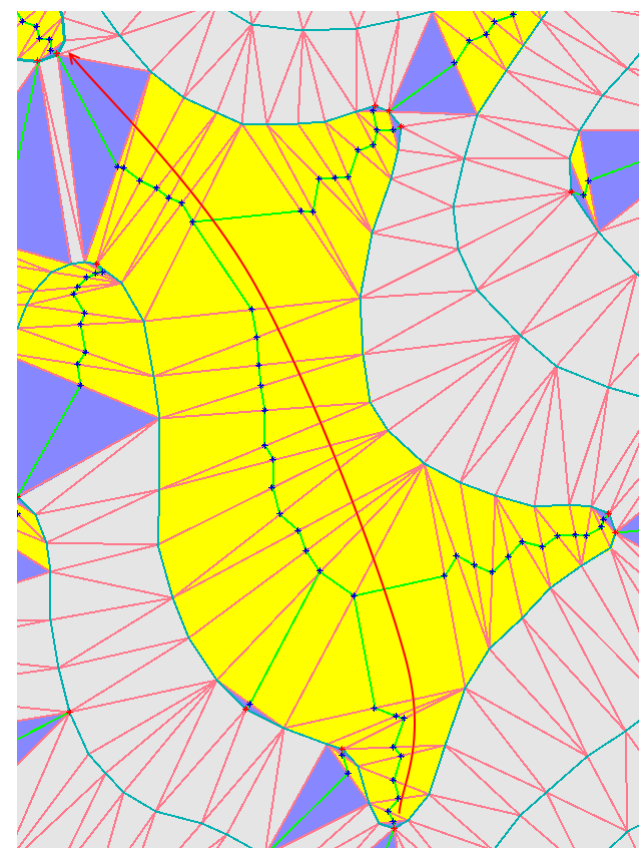

(a)

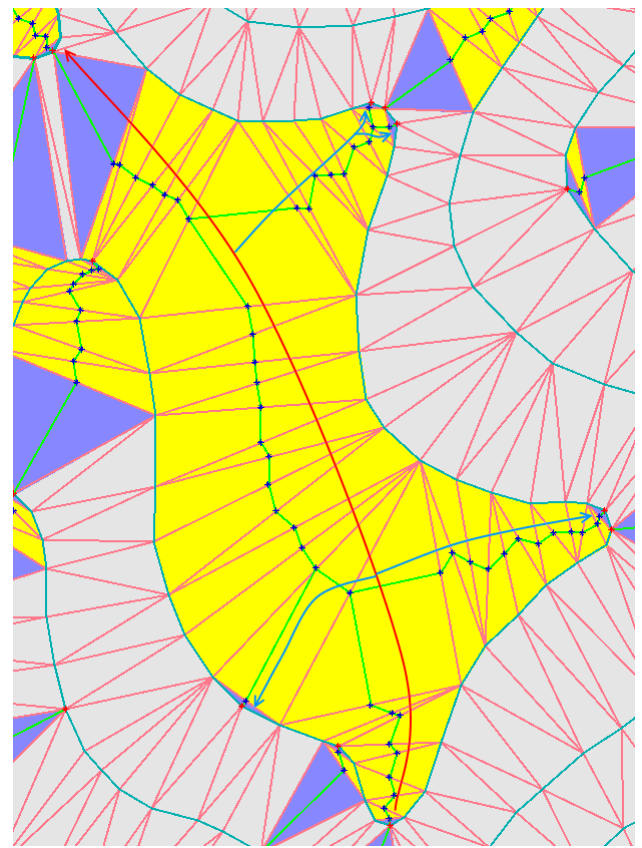

(b)

Figure 2.18 - Interpolation of critical points following branching paths: (a) first path (red) defined for interpolation of critical points. (b) branching paths (blue) with critical points to be interpolated next.

It is noteworthy that there are cases where the sequence of points to be interpolated contains both the initial and final points with the same elevation value. In this situation, the interpolation of the elevation values of all the critical points included in the path defined between these two points is not possible to be performed.

The procedure detailed so far, given by Barbalic and Omerbegovic (1999), does not deal with this problem, so this work proposes a solution that treats each case, thus defining an improvement on the procedure for removing flat areas from TINs. 
Firstly, it is necessary to identify the different cases where the initial and final points have the same elevation value. These cases are as follows:

a) The paths of triangles are inside a closed contour line that defines only flat triangles in its interior;

b) A path of triangles where the initial and final triangles are corner triangles with the points opposite to the critical edges of same elevation value, although these points have different elevation values in relation to the values of the flat triangles.

These two cases are illustrated in figure 2.19 with the same color scheme as given in previous figures. Figure 2.19(a) shows a closed flat area where any path defined between two corner triangles always traverses flat triangles, including the corner triangles, so a point of different elevation value is never reached. In figure 2.19(b), the corner triangles define a path with endpoints of same elevation value, even though of different values from the elevation values of the flat triangles.

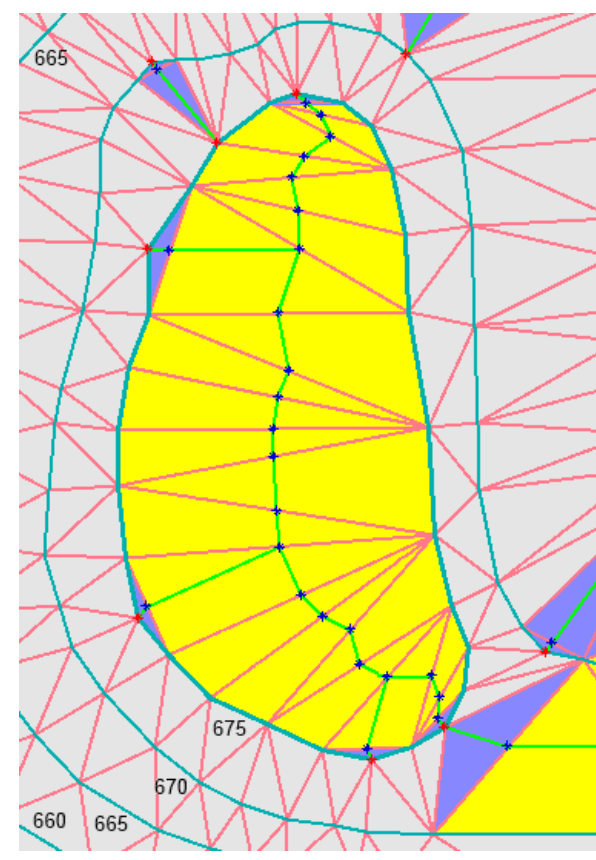

(a)

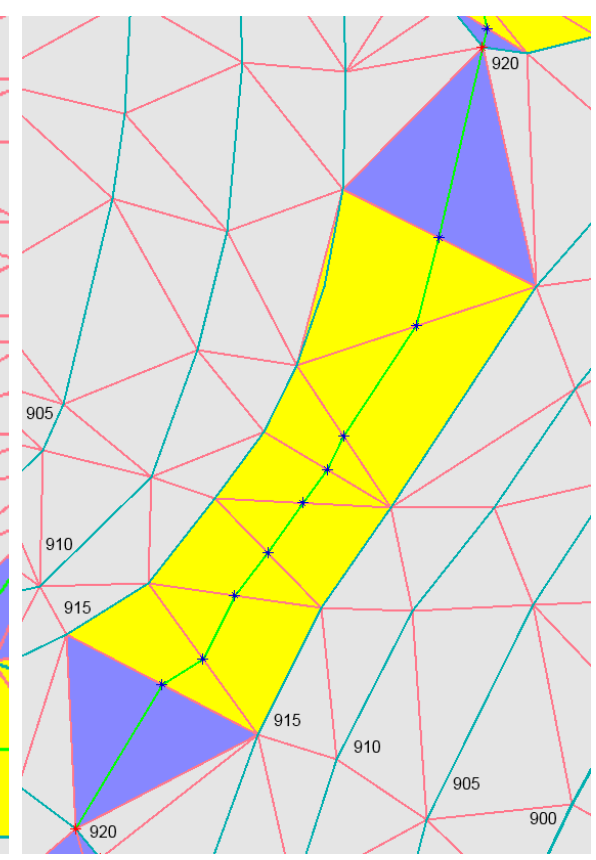

(b)

Figure 2.19 - Two different cases where the interpolation of elevation values of critical points is not possible: (a) closed contour line defines a closed flat area. (b) both corner triangles have the points opposite to the critical edges of same elevation value. 
The main difficulty in these cases is to determine whether the interpolation of all the critical points is upwards or downwards, i.e., whether the points have increasing or decreasing elevation values between the initial and final points. This problem is solved by getting information of the elevation values from neighboring contour lines (FREITAS et al., 2013).

Whenever a path of triangles is traversed, each triangle in the path is checked if it contains a non-critical edge defined by a contour line segment. Then the triangle adjacent to this edge is verified if the other point opposite to the edge has an elevation value lower or higher than the elevation value of the contour line that encloses the flat area, i.e., if it is located on another contour line.

This elevation variation between contour lines indicates whether the interpolated elevation values assigned to each critical point is increasing (neighboring contour lines values are lower) or decreasing (neighboring contour lines values are higher). Figure 2.20 exhibits the two previous cases, where neighboring contour lines indicate how the interpolation of the elevation values of critical points is performed. This is illustrated by the arrows going from one contour line to another.

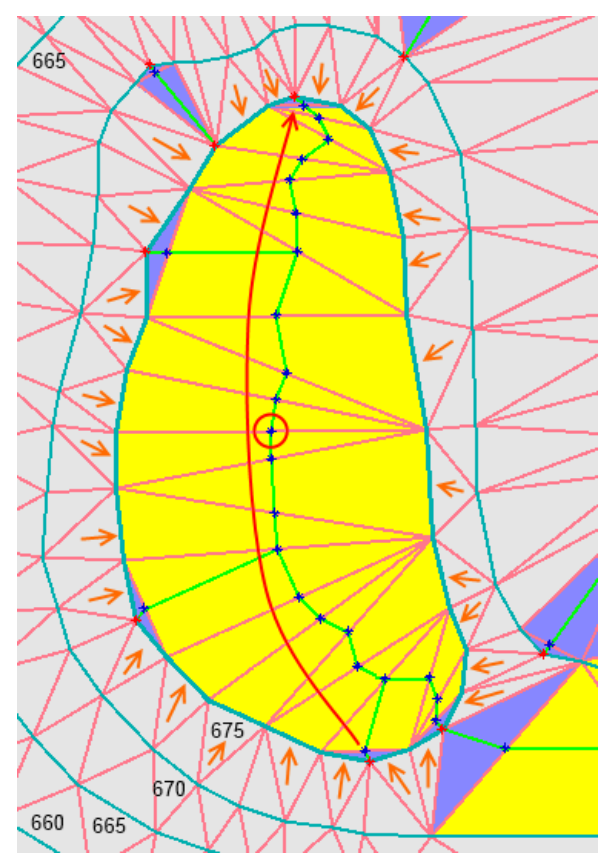

(a)

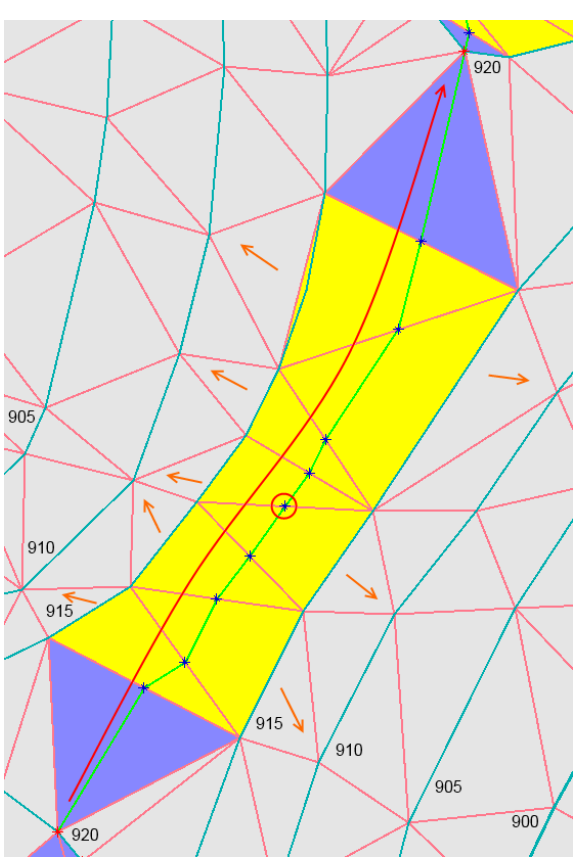

(b)

Figure 2.20 - Neighboring contour lines indicate how interpolation is performed: (a) interpolation of critical points define a hilltop at the point circled in red. (b) mean value between contour lines is assigned to critical point circled in red. 
In figure 2.20(a), the closed flat area is surrounded by a contour line of lower elevation, so the interpolation in this case is upwards. But it is still necessary to define what critical point inside the closed flat area has the highest elevation value, so that each path of flat triangles considers this point as its final point. This is done by selecting the half-way critical point of the first path, then assigning to this critical point an elevation value equal to the elevation of the flat area changed by the variation between contour lines, i.e., incremented or decremented by this amount. In this example, the elevation value is incremented resulting in a hilltop.

Figure 2.20(b) is an example of the other case. The neighboring contour lines of each flat triangle are lower than the elevation value of the flat area, but the corner triangles have points of higher elevation values. Once again, the half-way critical point of the first path is assigned an elevation value, but in this case, this value is equal to the mean value between the elevation value of the flat area and the elevation value of the points of the corner triangles that define the initial and final points.

\subsubsection{Pits}

Pits are usually considered as inaccurate data on DEMs because the majority of the depressions present in terrains are likely to be spurious features (SILVEIRA; OOSTRUM, 2007). In a TIN, pits are created as a result of the connections between edges of the triangulation at points where no neighboring point connected by a triangle edge has a lower elevation value. Figure 2.21 illustrates a pit highlighted in red together with the elevation values of each neighboring point.

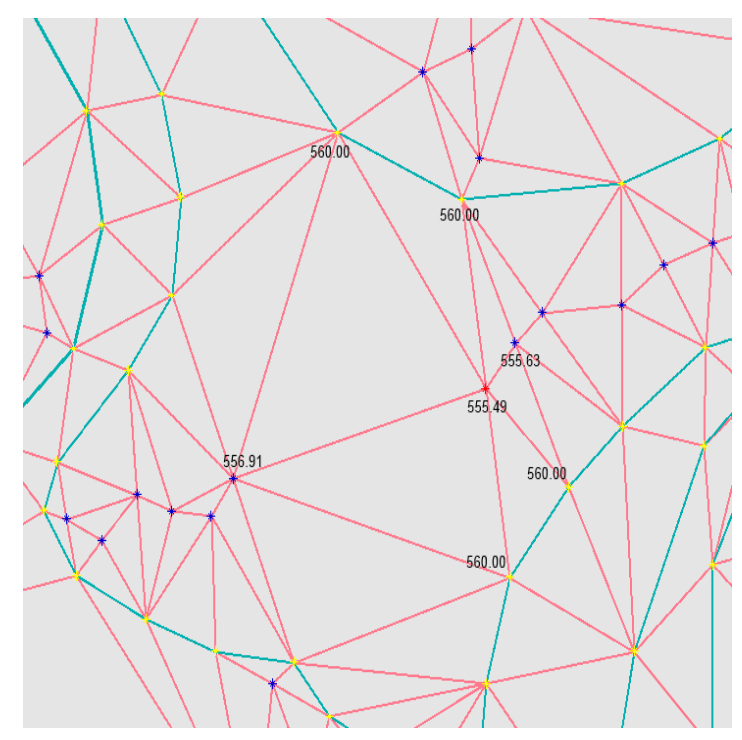

Figure 2.21 - Connections between triangles edges created a pit in the TIN 
Hydrological applications that use terrain models are often affected by the presence of pits because flows passing through these points do not follow any direction. This occurs since pits are local minima that hinder flow routing, thus determining discontinuities that must be avoided.

Several different schemes were devised for pit removal from TINs. A lifting approach (SILVEIRA; OOSTRUM, 2007; SILVEIRA, 2009) works by lifting the pit elevation to the lowest elevation value from its neighboring points, then including the neighbor of lowest elevation as part of a new pit consisting of more than one point. This lifting process is propagated until the pit is removed, so that it finishes only when some of the pit's neighboring points has a lower elevation value.

The aforementioned procedure is also known as a flooding technique for TINs. The main drawback of this approach is that all the points considered as part of the pit may define a flat area. Hence this pit removal procedure is likely to introduce an additional inconsistency into the terrain model after lifting the points, which must also be removed separately afterwards.

Another scheme also presented in Silveira and Oostrum (2007) and Silveira (2009) removes pits by lowering the elevation values of some points of the TIN that define a breaching path between the pit and another point of lower elevation. The technique also associates with each breaching path a cost to be minimized in order to modify the elevation values of each point as little as possible. This is a common solution for removing pits from regular grids known as carving process (SOILLE et al., 2003), where in this case the lowering of points is performed over the grid cells. An illustration of this technique performed on the TIN is shown in figure 2.22 .

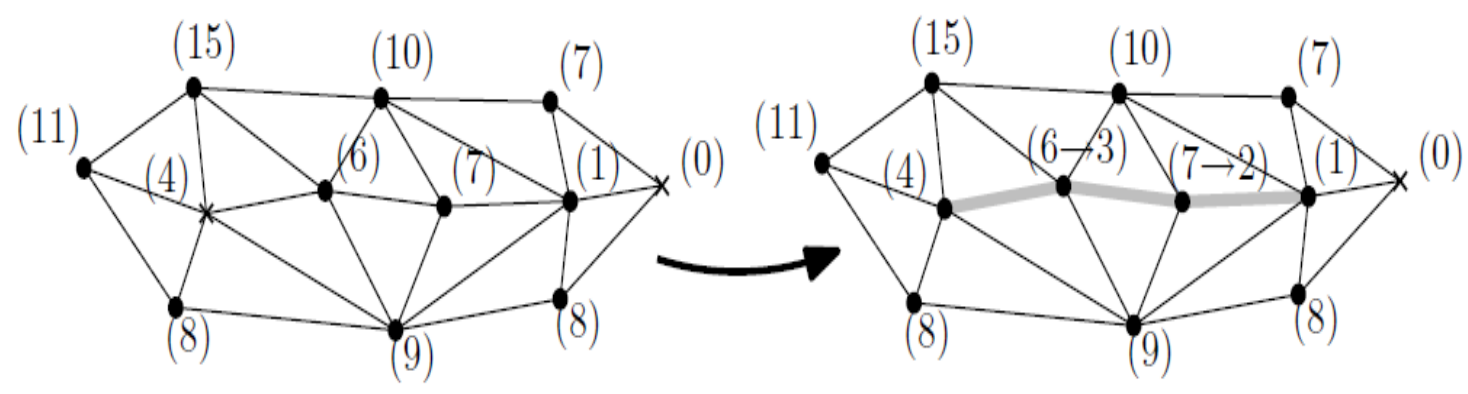

Figure 2.22 - Pit with elevation value 4 is removed by lowering the elevation of some points in a breaching path

SOURCE: Silveira and Oostrum (2007) 
In the present work, the procedure developed for pit removal is based on the definition of paths of points that start from the pit and traverses triangles edges until reaching another point of lower elevation, but without considering the cost of the paths. The reason for this is that the main purpose of removing pits from a terrain model is to avoid hydrological inconsistencies, so that flow directions are always defined independently of the new elevation values assigned to specific points that constitute the model.

Another modification in the procedure is that the elevation values of each point in the path are linearly interpolated between the initial and final points, in the same way that critical points are interpolated for removing flat areas.

It is worthy mentioning here that the surface topology is the primary concern when modifications and corrections are made in a terrain model, so if the elevation values of some points are changed and the most important topographic features of the surface are better represented, then the quality of the model is improved. Therefore these improvements are factors that directly influence all the subsequent results generated from the terrain model, specially when used in simulations of natural phenomena such as water-flow patterns over terrain surfaces.

Figure 2.23 exhibits how the pit shown in figure 2.21 is created after a retriangulation with the critical points for the removal of flat areas.

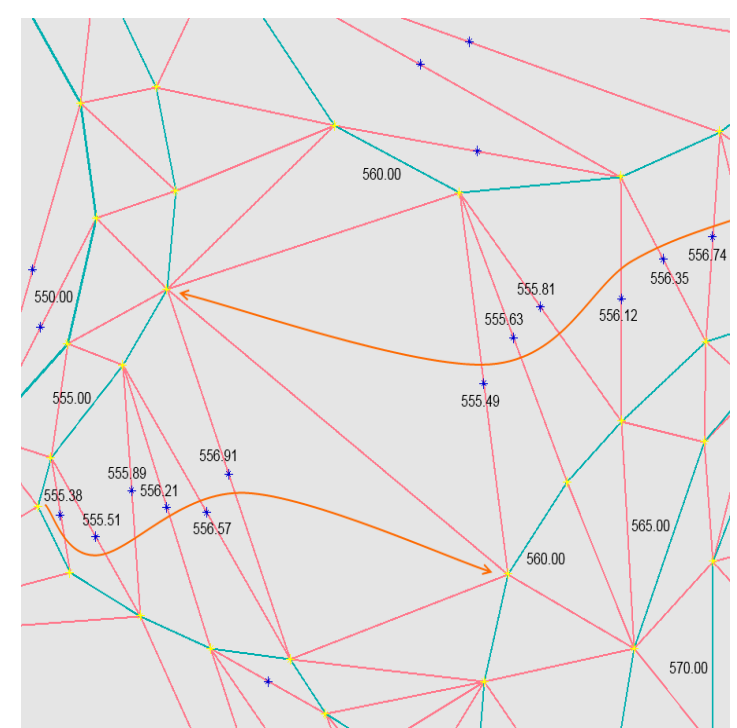

(a)

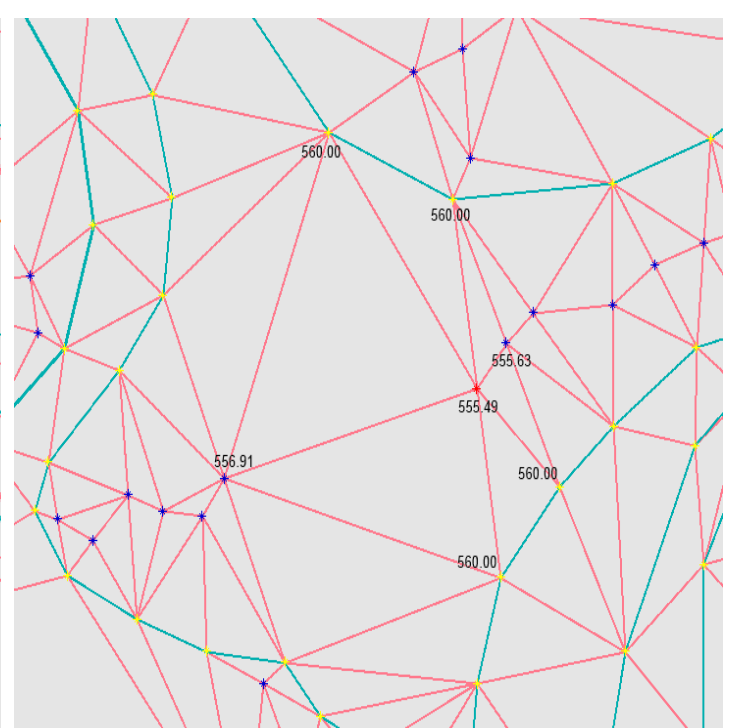

(b)

Figure 2.23 - Removal of flat areas creates a pit: (a) critical points with interpolated elevation values. (b) pit created after re-triangulation with critical points. 
The interpolated elevation values assigned to each critical point (blue) in this specific part of the terrain surface are calculated from two different paths of flat triangles (orange) which are not connected, although defined over the same region (figure $2.23(\mathrm{a})$ ). This occurs as the original triangulation does not properly represents the topology of the area between the contour lines, so that the interpolation processes performed on the two sets of points are independent of each other and, consequently, a pit (red) is created (figure 2.23(b)).

As an illustration of how the procedure for pit removal works, figure 2.24 exhibits paths of points generated at different steps for the same region. These paths (light green) are defined from a breadth-first search initiated at the pit (red), then following through each triangle edge to all unvisited neighboring critical points (blue). Thus if a point of lower elevation value relative to the pit elevation is reached, the path for removing the pit is then defined and the elevation values of each point in the path are linearly interpolated between the elevation values of the pit and the point of lower elevation. The red arrows in figures 2.24(e) and 2.24(f) indicate the path that contains the points where the elevation values are re-interpolated.

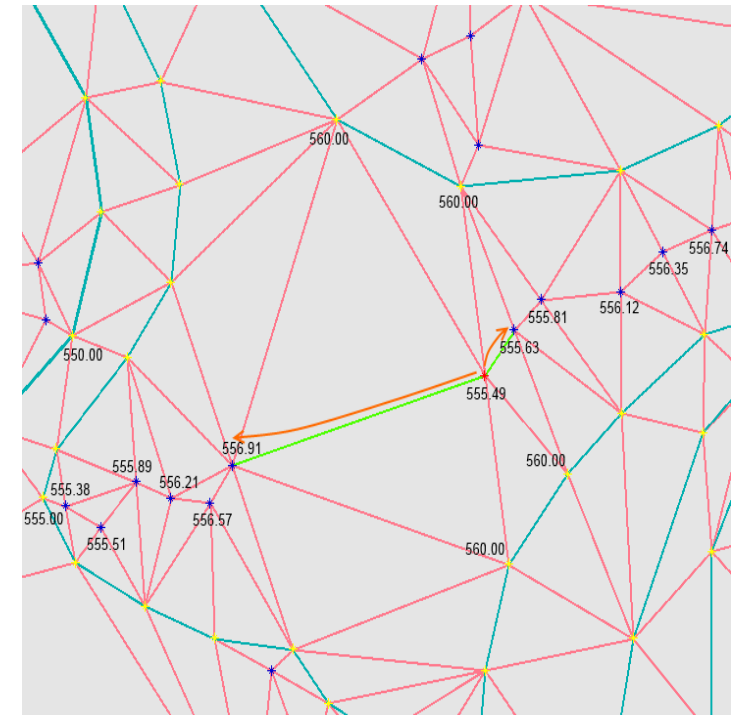

(a)

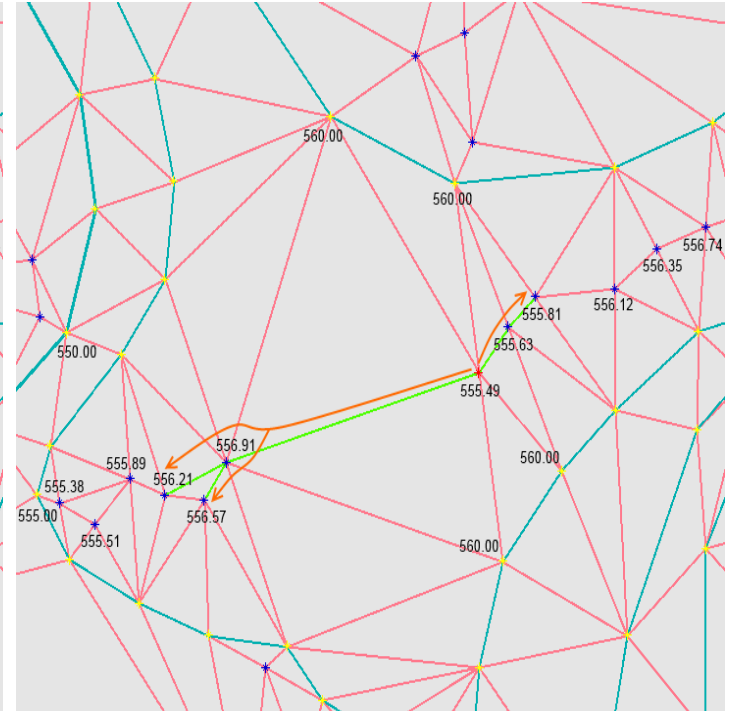

(b)

Figure 2.24 - Paths of critical points for pit removal: (a) paths are initiated at the pit (red). (b) points reached have higher elevation values. (continue) 


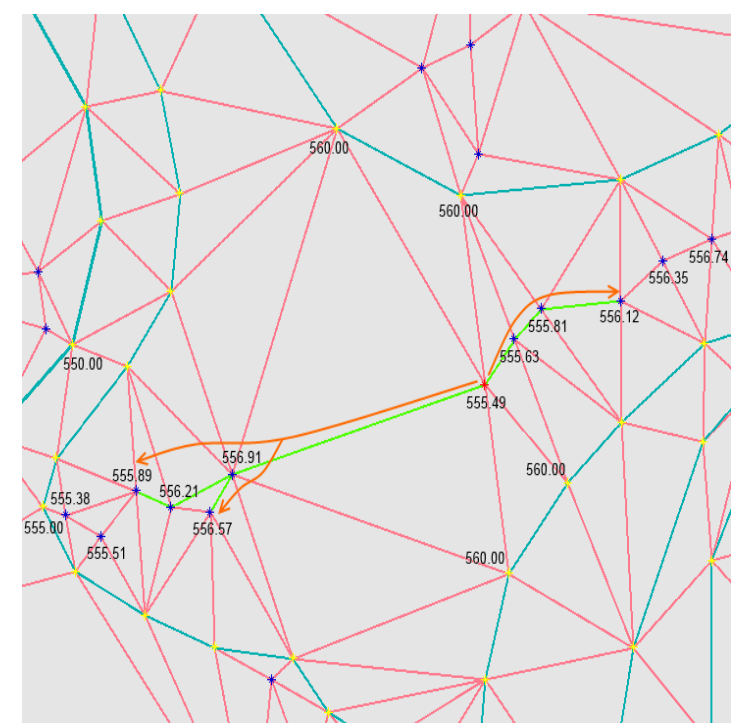

(c)

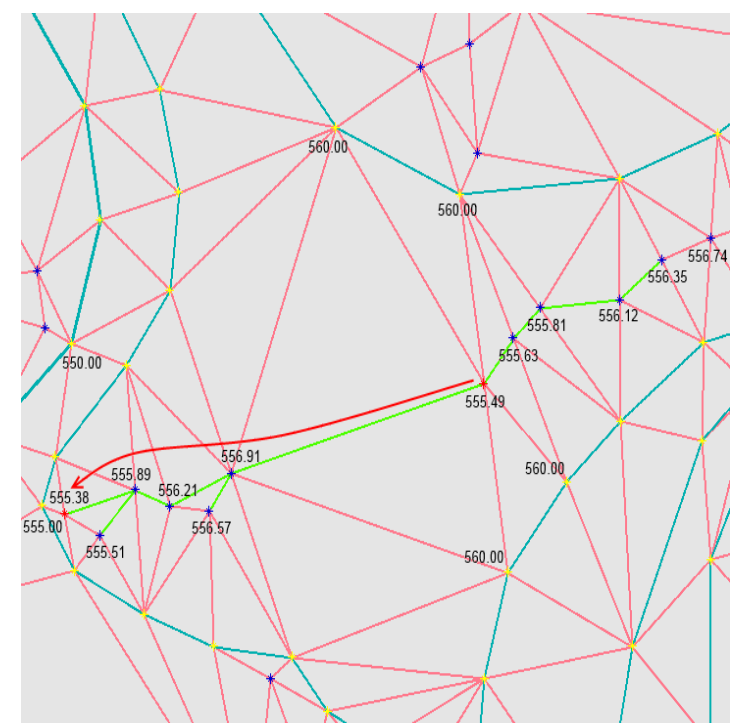

(e)

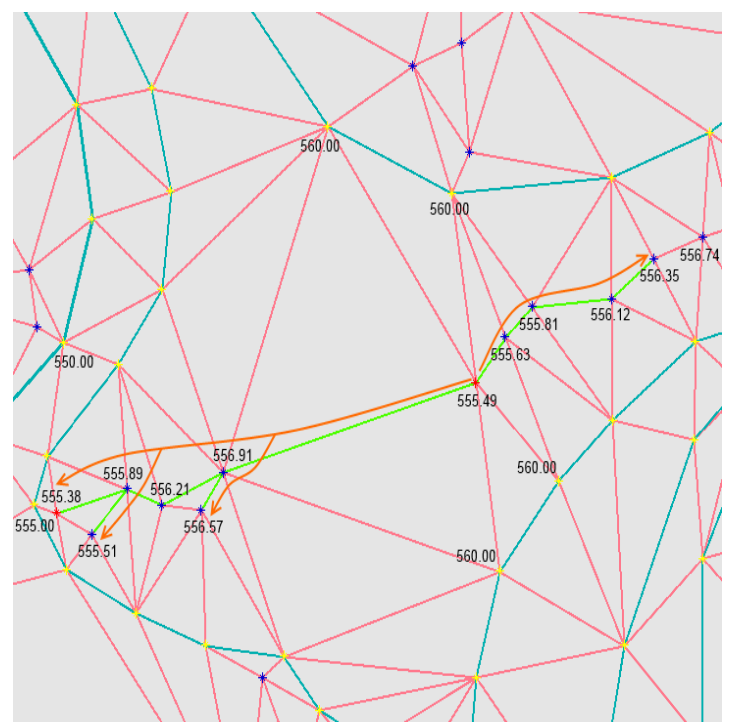

(d)

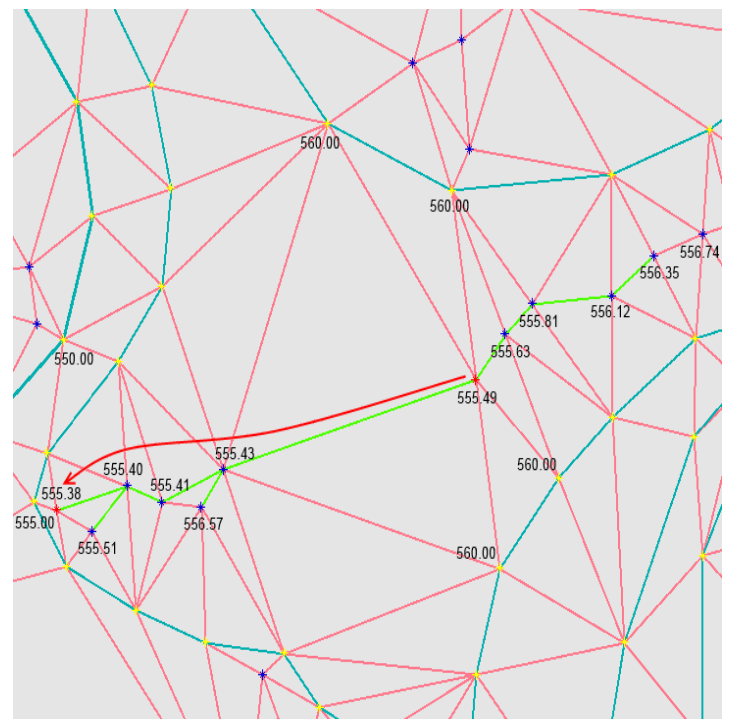

(f)

Figure 2.24 - Paths of critical points for pit removal: (c) paths of critical points still being traversed. (d) critical point of lower elevation (red) is reached. (e) path of critical points with elevation values to be re-interpolated is indicated by the arrow. (f) critical points with re-interpolated elevation values. (conclusion)

One important detail about the pit removal procedure is that the paths of points defined by traversing triangles edges depend on the type of point the pit is, i.e., either a contour line point or a critical point. As described earlier, critical points are points inserted into the triangulation for removing flat areas, so that if the pit is a critical point, its elevation value was previously calculated from an interpolation process performed in a sequence of critical points. Thus the critical point that defines the 
pit as well as other critical points may have their elevation values re-interpolated.

In the case that the pit is a critical point, the paths of points are defined only by critical points reached from the pit, as shown in the example of figure 2.24. Thus contour line points, if reached, are just checked for a lower elevation value which then determinines a final point for interpolation, otherwise these points are not included in the path.

Considering the other case, when the pit is a contour line point, the paths of points to be defined may include both critical points as well as contour line points, but the contour line points must have an elevation value equal to the pit elevation in order to be included in the path, i.e., these points must be on the same contour line reached by traversing triangles edges from the pit. Apart from this difference, the procedure for removing the pit is performed in the same way as mentioned before. 


\section{HYDROLOGICAL STRUCTURES}

Hydrological structures such as drainage networks and watersheds present important applications in hydrology since drainage networks indicate the main streams of water over a terrain surface while watersheds define the regions from where the water flows contribute to specific streams of the drainage network. In addition, both structures can be generated by the definition of drainage paths from a terrain model used as an essential source of information to derive hydrological patterns.

To a large extent, the main use of these structures are commonly associated with hydrological models developed to address several problems in water resources management, which usually depend on terrain information. For instance, the DHSVM (Distributed Hydrology Soil Vegetation Model) (WIGMOSTA et al., 2002) has been used to study interactions between climate and hydrology, potential impacts of climate change on water resources, forest management activities on watershed processes and runoff estimation with transport of water, sediments, chemicals and nutrients. Similarly, the MGB-IPH distributed hydrological model (COLLISCHONN et al., 2007) focuses on the rainfall-runoff analysis in large-scale basins.

\subsection{Regular grid hydrological structures}

According to Collischonn (2001), the drainage area is a measure that integrates either the surface or the subsurface flows of water from a point in the terrain. Specifically, flow directions defined on regular grids indicate how the drainage areas are accumulated from cell to cell, so that the drainage networks are represented by all the cells that store a value equal to or greater than a given threshold. Figure 3.1 illustrates how each grid cell contributes to the drainage area accumulated in subsequent cells by following the flow directions.

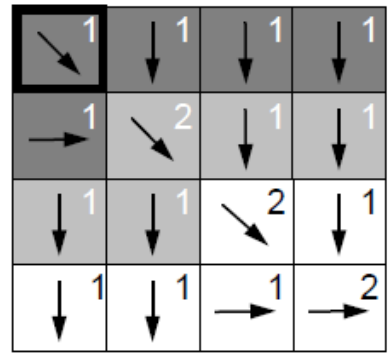

Step 1: Cell 1

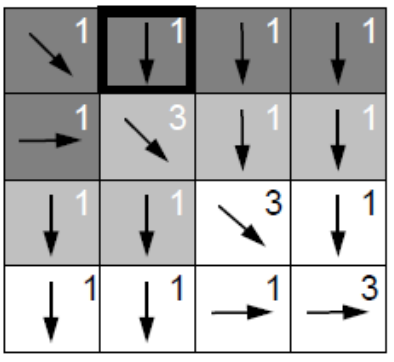

Step 2: Cell 2

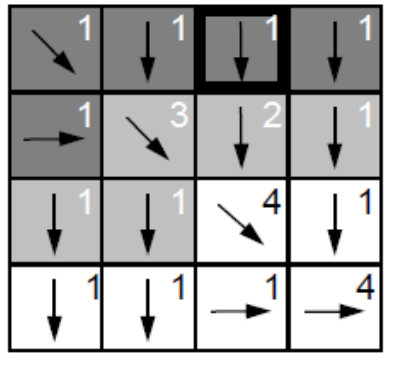

Step 3: Cell 3

Figure 3.1 - Accumulated flows over the regular grid SOURCE: adapted from Collischonn (2001) 
Moreover, the flow directions are also used to delineate watersheds either from a particular grid cell, which is considered as the watershed's outlet point, or from each drainage segment of the drainage network, resulting in different subwatersheds. Thus all the cells reached from a specific point in the drainage network by following the flow directions in the opposite way are then included as part of region that defines the watersheds.

The extraction of drainage networks and watersheds from regular grids were extensively considered in several works (FOWLER; LITTLE, 1979; O'CALLAGHAN; MARK, 1984; JENSON; DOMINGUE, 1988; ABREU et al., 2012) with a wide range of applications regarding water resources. An example of these hydrological structures can be visualized in figure 3.2, where both structures were generated from a regular grid defined by the SRTM surface model (JARVIS et al., 2008).

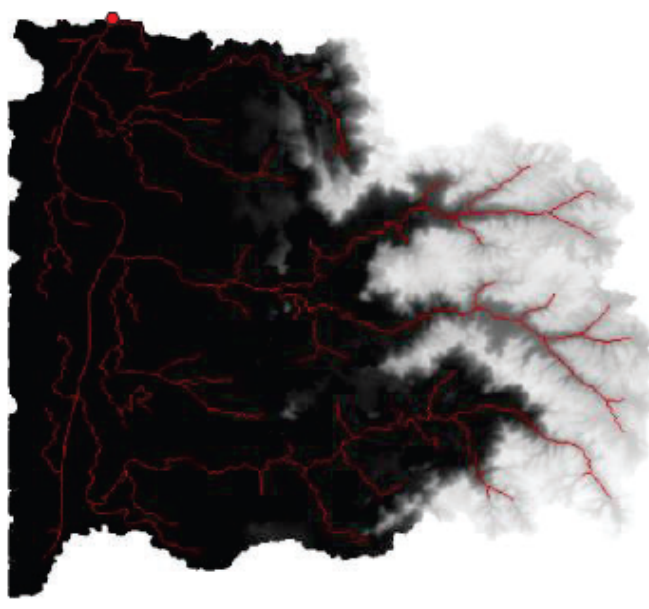

(a)

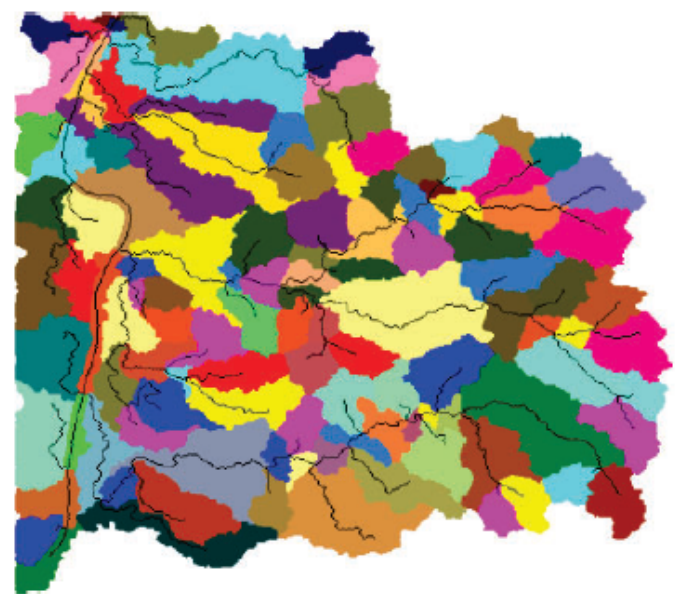

(b)

Figure 3.2 - Drainage network and watersheds defined from a regular grid SOURCE: Abreu et al. (2012)

As already mentioned, flow directions defined over regular grids are restricted to only eight different directions because each cell has eight neighboring cells. For this reason, hydrological results derived from regular grids can be improved with an increase on the grid resolution since more points define the terrain model (although, depending on the region, it is likely that the model presents large areas of redundant data) and, consequently, more flow directions are assigned over the terrain surface, so that the resulting drainage networks and watersheds determine a more detailed representation of the real-world hydrological patterns. 


\section{$3.2 \quad$ TIN hydrological structures}

Some authors investigated and developed techniques to calculate drainage paths directly from TIN terrain models. One approach detailed by Silfer et al. (1987) determines how water should be routed across the surface of a TIN distinguishing from two different conditions between triangles. These two conditions are listed below, where IIO and IOO represent In-In-Out and In-Out-Out cases, respectively. So the two conditions are as follows:

- Flow enters two sides of a triangle and exits from one side (IIO case);

- Flow enters one side of a triangle and exits from two sides (IOO case).

The flow direction is given by a vector normal to the triangle, so that when the vector is projected onto the $x y$ plane, the $x$ and $y$ components of the projected vector define a line of maximum slope and thus the direction that the water flows across the triangle (SILFER et al., 1987). The normal vector of a triangle and its respective projected vector are depicted in figures 3.3(a) and 3.3(b) below.

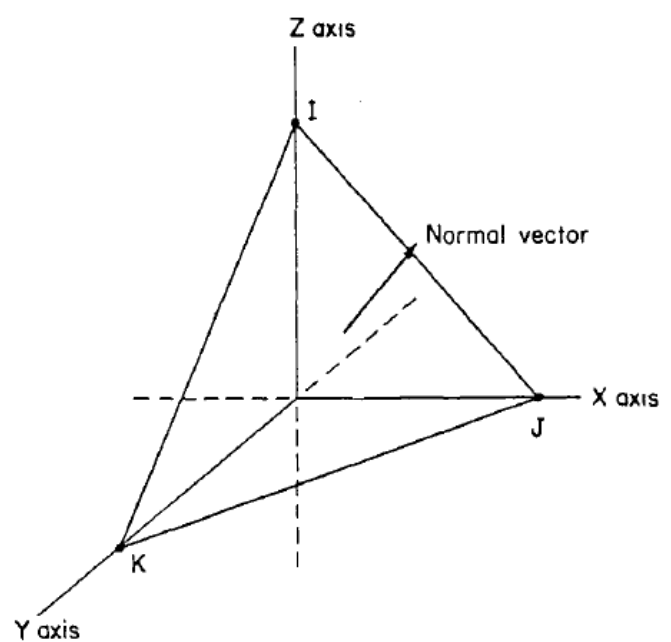

(a)

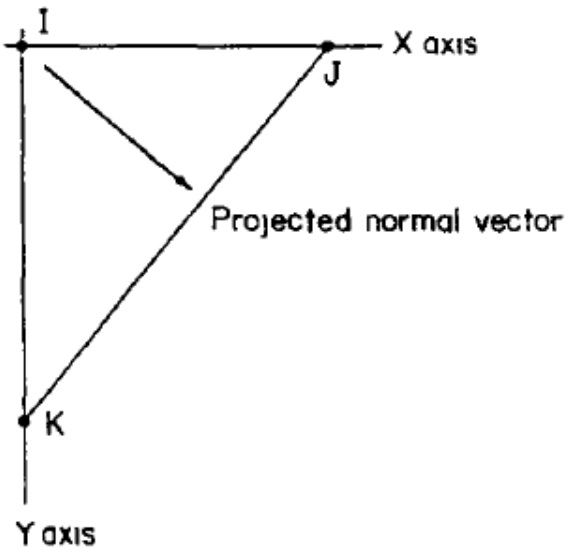

(b)

Figure 3.3 - Vectors defined from a triangle: (a) normal vector. (b) projected vector. SOURCE: Silfer et al. (1987)

The projected vector determines the slope aspect of the triangle and its base point is either considered the triangle point of highest elevation value, in an IIO case, or the triangle point of lowest elevation value, in an IOO case. These two cases are 
distinguished by the slope aspect as the projected vector can be located either inside or outside the triangle. Figure 3.4 illustrates both IIO and IOO flow cases with the slope aspect vector included.

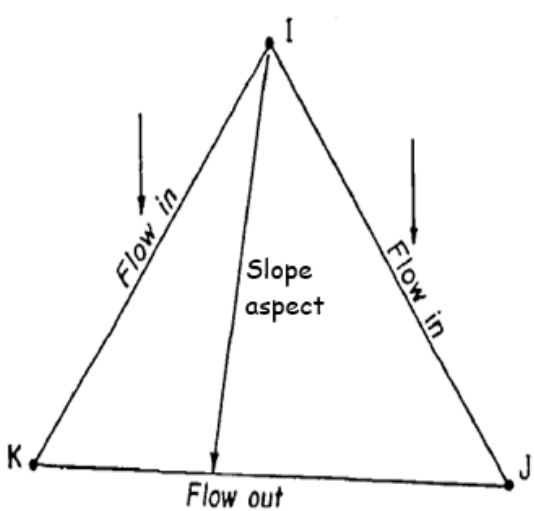

(a)

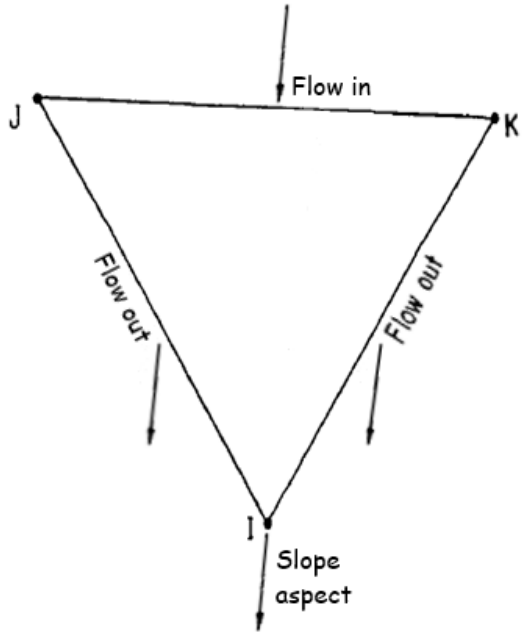

(b)

Figure 3.4 - In-In-Out and In-Out-Out flow cases: (a) IIO case. (b) IOO case. SOURCE: adapted from Silfer et al. (1987)

Although this technique considers flow directions given by specific vectors calculated from each triangle, it does not specify how this information can be used in order to generate drainage networks over the TIN model. In addition, as the flows are defined from only two different cases between triangles, this determines a restriction that hinders the generation of more natural water-flow patterns.

A method described by Jones et al. (1990) contains many important concepts considering the flow directions over the TIN given by the gradient of the plane defined from each triangle. This method includes the fundamental techniques used in this work as a basis for the generation of drainage paths from the terrain model represented by the TIN. The main details of its formulation and procedures will be presented in the next section.

Another approach from a more recent work by Tsirogiannis (2011) defines trickle paths by tracing a sequence of edges and vertices, called an EV-sequence, considered as a set of terrain features determined from the paths of steepest descent calculated over the TIN, which is fundamentally the method given by Jones et al. (1990). 
That work focuses on how to avoid the explicit computation of all the intersections with triangles edges while tracing a trickle path of a point $p$ on the TIN. This is achieved by marking each terrain feature as already crossed at the first time it is encountered, so that if one terrain feature is marked and it leads to another one not marked yet, it then defines a cycle inside of a trickle path and thus all the points that are out of the cycle do not cross any of the terrain features previously encountered.

Figure 3.5 exhibits a cycle of terrain features from an EV-sequence of the trickle path calculated from the point $p$. This sequence also includes the point $q$, although this point is not part of any terrain feature already crossed. The cycle finishes where the terrain feature $f_{i}$ is equal to $f_{j}$, with $i<j$, since from their respective intersections $r_{i}$ and $r_{j}$ the trickle path next intersects different terrain features. Point $q$ determines the terrain feature $f_{k}$ located out of the cycle, with $k>j$.

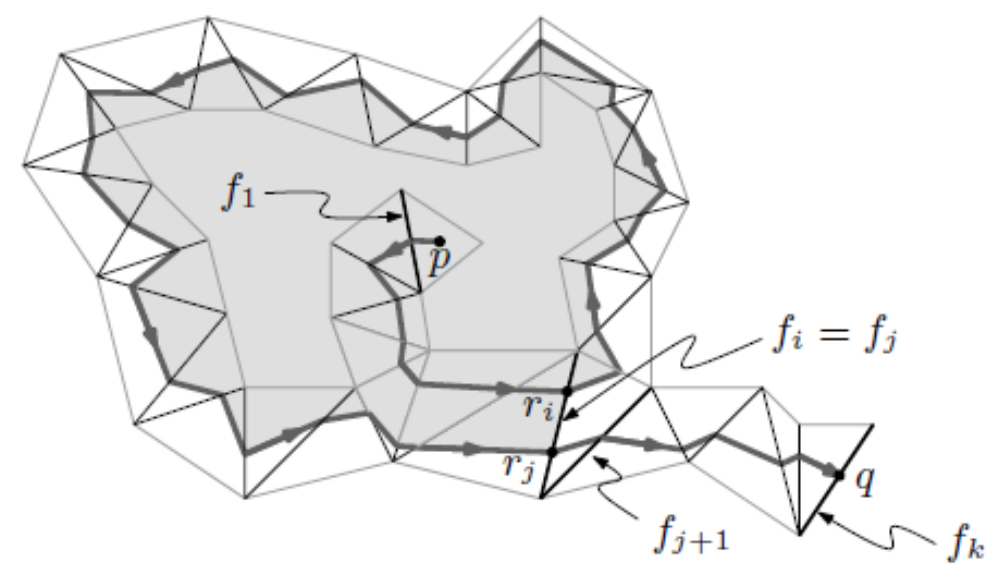

Figure 3.5 - Trickle path of a point $p$ includes a cycle of terrain features SOURCE: Tsirogiannis (2011)

Cycles determined from a trickle path are not likely to occur in a terrain surface except when the triangles are traversed over a flat area where the flow directions are not defined. So depending on how the flow directions are calculated over the TIN, the trickle paths may possibly return to previously-crossed terrain features.

Trickle paths starting at initial points selected from the TIN can produce strip maps (TSIROGIANNIS, 2011) comprised of lines of steepest descent, which constitute the flows of water over the terrain surface. Figure 3.6 illustrates a strip map with many trickle paths, although not extended over the bottom part as it defines a flat area where the flow directions are undefined. 


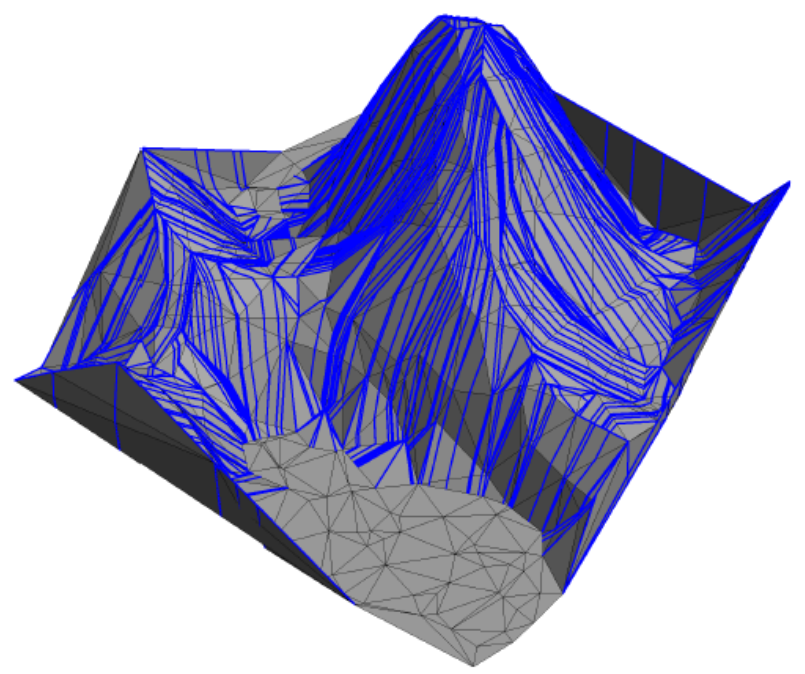

Figure 3.6 - Strip map over the TIN

SOURCE: Tsirogiannis (2011)

As this approach considers the flow directions given by the lines of steepest descent calculated from the triangles, the flows are not restricted to specific directions or limited to follow only through triangles edges, so that all the flows produce more representative water-flow patterns. However, it is not defined how the trickle paths traced over the TIN can be used to generate drainage networks.

Even though the aforementioned works provide many details about drainage patterns derived from TIN terrain models, it is not specified how the drainage paths can be used to calculate accumulated flows for the generation of drainage networks and their associated watersheds. The methodology proposed in this work describes the fundamental concepts of paths of steepest descent given by gradient vectors and how these paths define a graph structure which allows the generation of both drainage networks and watersheds from accumulated flows.

\subsubsection{Gradient method}

Terrain models represented by TINs can be considered as continuous piecewise linear functions generated from the set of points that define the terrain surface. Usually, the flow of water calculated from a terrain model is a purely geometrical problem and does not take into account aspects such as soil type and land cover (TSIROGIANNIS, 2011). However, this exclusively geometrical representation of both the terrain surface and the flow model constitutes a good approximation of the real-world terrain structures and their associated drainage patterns. 
As previously mentioned, the generation of drainage paths in this work is based on the flow directions given by gradient vectors (JONES et al., 1990). Each triangle from the TIN defines a plane surface that passes through its three vertices and the gradient vector is then calculated from the triangle plane as described next.

The linear plane defined by a triangle with vertices $v_{1}=\left(x_{1}, y_{1}, z_{1}\right), v_{2}=\left(x_{2}, y_{2}, z_{2}\right)$ and $v_{3}=\left(x_{3}, y_{3}, z_{3}\right)$ is given by equation 3.1. The coefficients $A, B, C$ and $D$ of the plane equation (equations 3.2, 3.3, 3.4 and 3.5, in this order) are calculated from a cross product between the vectors $\overrightarrow{v_{1} v_{2}}$ and $\overrightarrow{v_{1} v_{3}}$, which are defined from the vertices $v_{1}$ and $v_{2}$ as well as $v_{1}$ and $v_{3}$, respectively.

$$
\begin{gathered}
A x+B y+C z+D=0 \\
A=y_{1}\left(z_{2}-z_{3}\right)+y_{2}\left(z_{3}-z_{1}\right)+y_{3}\left(z_{1}-z_{2}\right) \\
B=z_{1}\left(x_{2}-x_{3}\right)+z_{2}\left(x_{3}-x_{1}\right)+z_{3}\left(x_{1}-x_{2}\right) \\
C=x_{1}\left(y_{2}-y_{3}\right)+x_{2}\left(y_{3}-y_{1}\right)+x_{3}\left(y_{1}-y_{2}\right) \\
D=-A x_{1}-B y_{1}-C z_{1}
\end{gathered}
$$

Rewriting the plane equation 3.1 with the elevation variable $z$ as a function of the coordinate variables $x$ and $y$, it results in equation 3.6 below.

$$
z=f(x, y)=-\left(\frac{A}{C} x+\frac{B}{C} y+\frac{D}{C}\right)
$$

At this point, by applying the gradient operator to the above function, which is defined by equation 3.7 with the partial derivatives $\frac{\partial f}{\partial x}$ and $\frac{\partial f}{\partial y}$, the gradient vector then points in the direction of steepest ascent. Thus by calculating the negative gradient of the function $z=f(x, y)$ since it gives the direction of steepest descent, the gradient vector projected onto the $x y$ plane is defined by equation 3.8 . The vector components $\frac{A}{C}$ and $\frac{B}{C}$ determine the flow direction to be followed from a point in a triangle, representing the variations in the $x$ and $y$ directions, respectively.

$$
\begin{aligned}
& \nabla f=\frac{\partial f}{\partial x} \vec{i}+\frac{\partial f}{\partial y} \vec{j} \\
& -\nabla f=\frac{A}{C} \vec{i}+\frac{B}{C} \vec{j}
\end{aligned}
$$




\subsubsection{Drainage paths}

Each drainage path generated over the TIN terrain model begins at some point located at an initial triangle always following the path of steepest descent, i.e., the direction given by the gradient vectors from the planes of the triangles crossed while the drainage path is traced (JONES et al., 1990). It is worthy mentioning that in this work each flow direction is unique for any point on the terrain surface, which is the standard flow model used in digital terrain analysis (TSIROGIANNIS, 2011).

Figure 3.7 illustrates a drainage path traced from gradient vectors through the triangles beginning at the starting point $a$. Each triangle contains interpolated elevation values calculated from its respective linear plane indicating how the directions are followed.

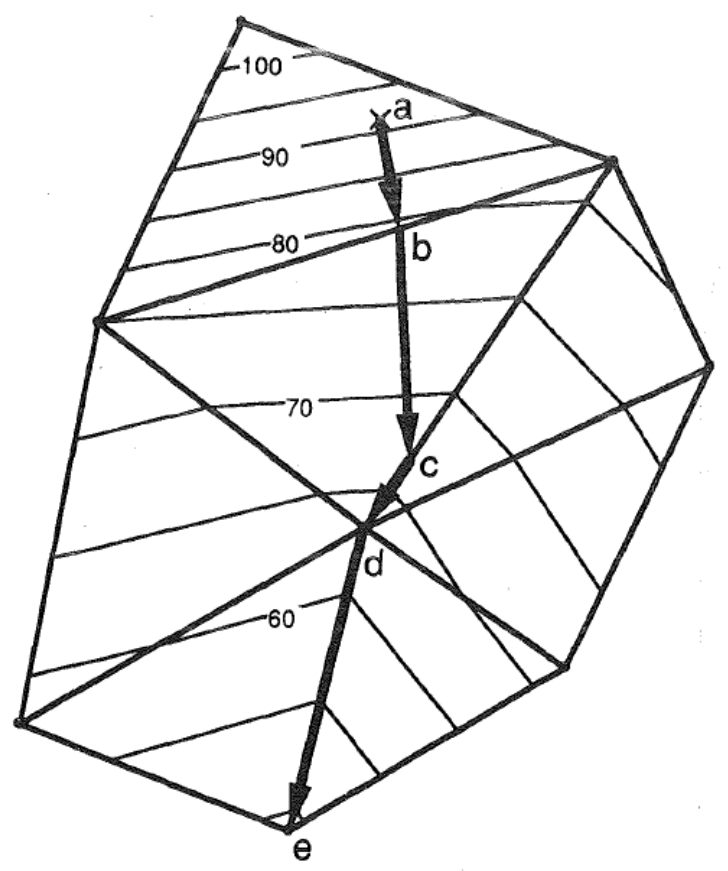

Figure 3.7 - Drainage path over the TIN SOURCE: Jones et al. (1990)

When a drainage path is traced, different situations can occur regarding the intersections between gradient vectors and triangles edges. Each situation is related to whether the intersection is either along an edge or exactly in a vertex, and also whether the gradient vectors of adjacent triangles point to each other or not. These situations are summarized as follows: 
- The gradient vector of a triangle intersects one of its edges, not in a vertex. In this case, the gradient vector of the adjacent triangle opposite to the intersected edge is checked if:

a) It points away from the current triangle, so the drainage path continues across the adjacent triangle in the same way it follows through the current triangle;

b) It points back to the current triangle, thus forming a channel edge, so the drainage path continues along the common edge between the triangles towards the vertex of lowest elevation value.

- The gradient vector of a triangle intersects one of its edges exactly in a vertex. Then all the edges and triangles incident to the vertex are checked if they meet some specific conditions in order to find the lowest elevation point reached from the vertex:

a) Each edge is checked if it is a channel edge, i.e., both gradient vectors of the adjoining triangles by the edge point to each other, and also if the other vertex of the edge has a lower elevation value relative to elevation of the current vertex;

b) Each triangle is checked if there is an intersection between its gradient vector based at the current vertex and its edge opposite to the vertex, so that the gradient vector lies between the other two edges. In addition, this intersection must also have an elevation value lower than the elevation value of the current vertex.

Considering all these conditions, after the point of lowest elevation value is found, the drainage path continues either along an edge to another vertex, in the case of a channel edge (figure 3.8(a)), or across a triangle (figure 3.8(b)), and the process is repeated for each vertex or triangle traversed while the drainage path is traced.

Therefore there are only two distinct procedures that generate the drainage paths since the flow can continue either from a vertex or from an edge, and each procedure calls the other one or itself recursively. 


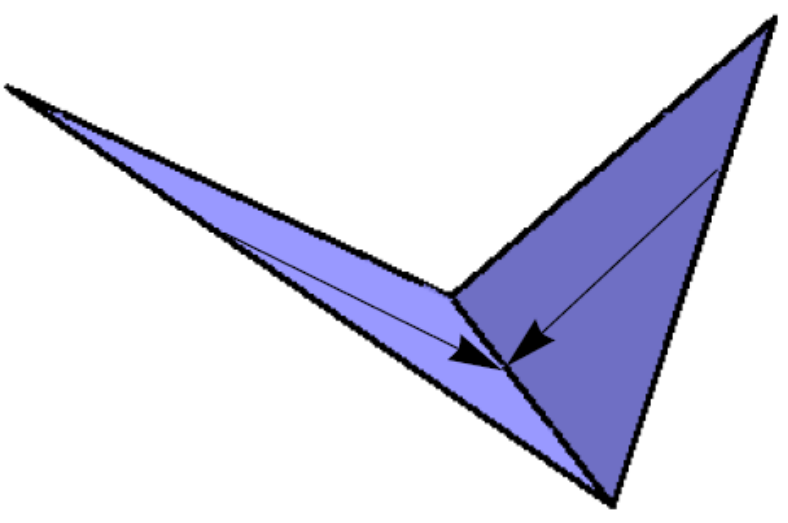

(a)

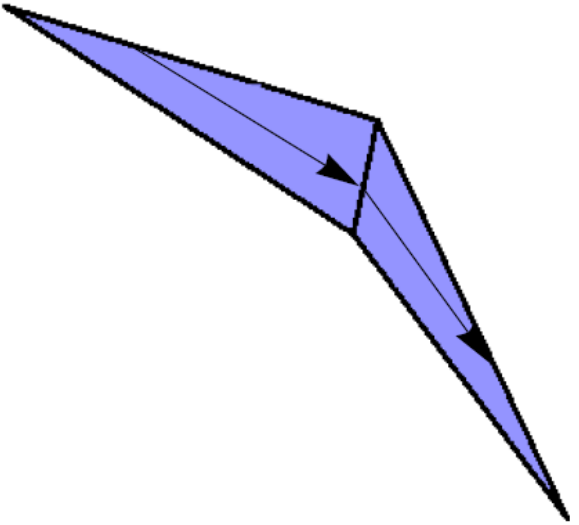

(b)

Figure 3.8 - Flows over the TIN: (a) Flow continues along a channel edge. (b) Flow continues across a triangle.

SOURCE: Tsirogiannis (2011)

Figure 3.9 shows two previous flows, one passing across a triangle and the other along an edge. The current flow leaves vertex $i$ of the triangle $i j k$, where its gradient vector lies between the edges $i j$ and $i k$, so that it intersects the edge $j k$ and the drainage path will then continue to be traced from the point of intersection with the edge.

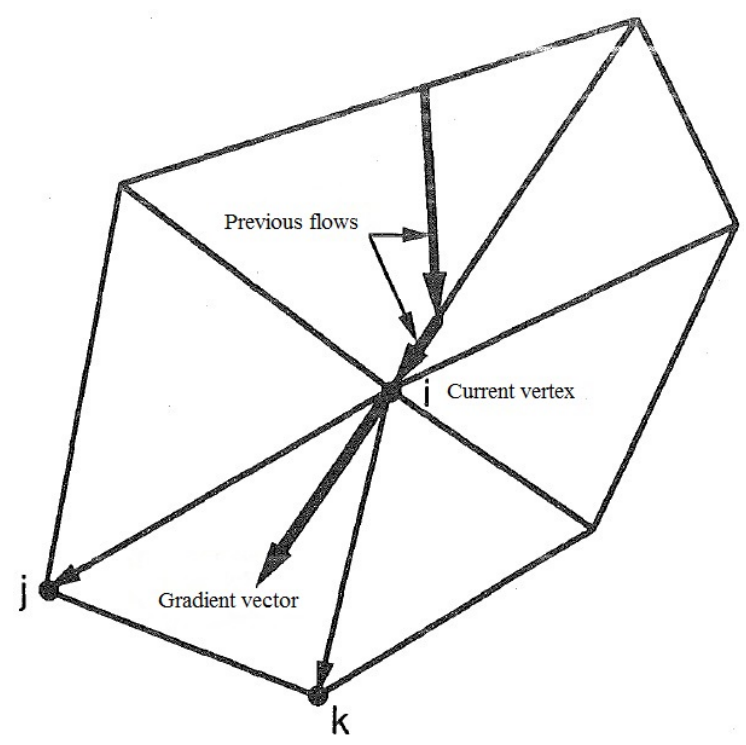

Figure 3.9 - Current flow continues from a vertex across a triangle SOURCE: Jones et al. (1990) 
One important observation to be made about the procedure for generating drainage paths is that by following paths of steepest descent, either across the triangles or along the edges, the flow directions calculated from the TIN are better approximations for the flows of water defined over a terrain surface, instead of using simplified models that only consider flows along the TIN edges (TSIROGIANNIS, 2011). Flows calculated from simplified models are restricted to a subset of the TIN surface rather than considering it in its entirety, which can affect the quality of the drainage paths.

\subsubsection{Drainage graph}

The procedure described in the previous section for constructing drainage paths can be performed by selecting any point on the TIN surface as a starting point. In this work, the points selected as starting points are the triangles centroids defined by each barycenter, which approximately represents the elevation of the triangles.

Figure 3.10 exhibits part of a triangulation where the elevation of each triangle centroid is calculated as the average value from the elevation values of its three vertices. Points in yellow define contour lines, blue points are critical points with interpolated elevation values and triangles centroids are in red.

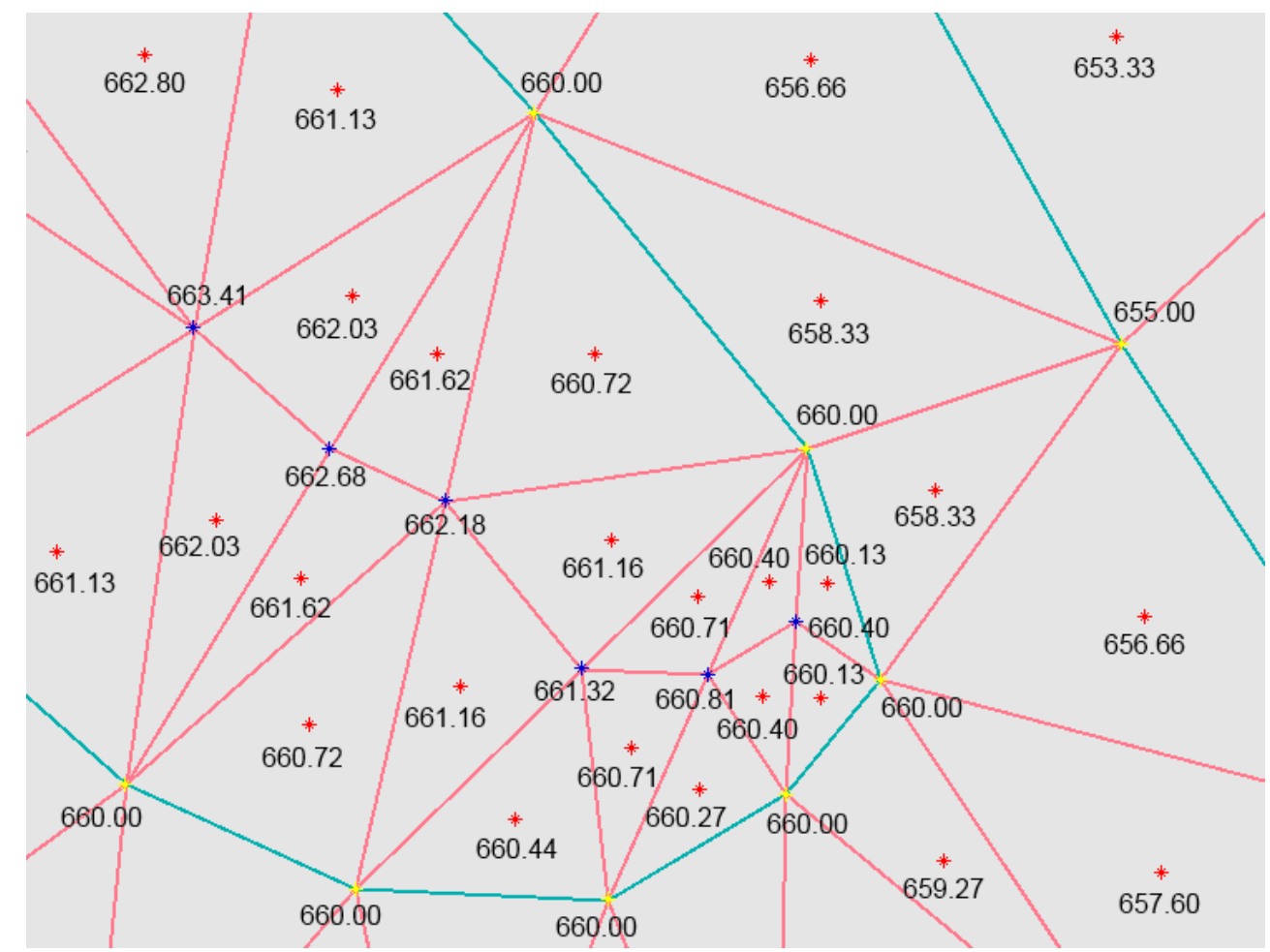

Figure 3.10 - Triangles centroids (red) represent the elevation of each triangle 
In the present work, the elevation of each starting point is considered as a priority value associated to the point. This priority value defines the order in which all the points are processed for the calculation of the drainage paths, beginning at the triangles centroids ordered from highest to lowest elevations. An important aspect of this procedure is that whenever a drainage path is traced and it reaches a triangle where another drainage path is already defined, then the current path is connected to the existing path. The procedure terminates after each starting point has been processed and all the drainage paths have been connected.

Steps of this procedure can be visualized in figure 3.11, where triangles centroids are in red and each intersection between triangles edges and drainage paths are in cyan. The first drainage paths are traced beginning at the triangles centroids of higher elevations, then all the other drainage paths that begin at points of lower elevations are traced in subsequent steps and connected to the outflow of the triangles crossed by the drainage paths previously defined. At the end, this procedure generates a drainage structure which represents the flow directions over the TIN surface.

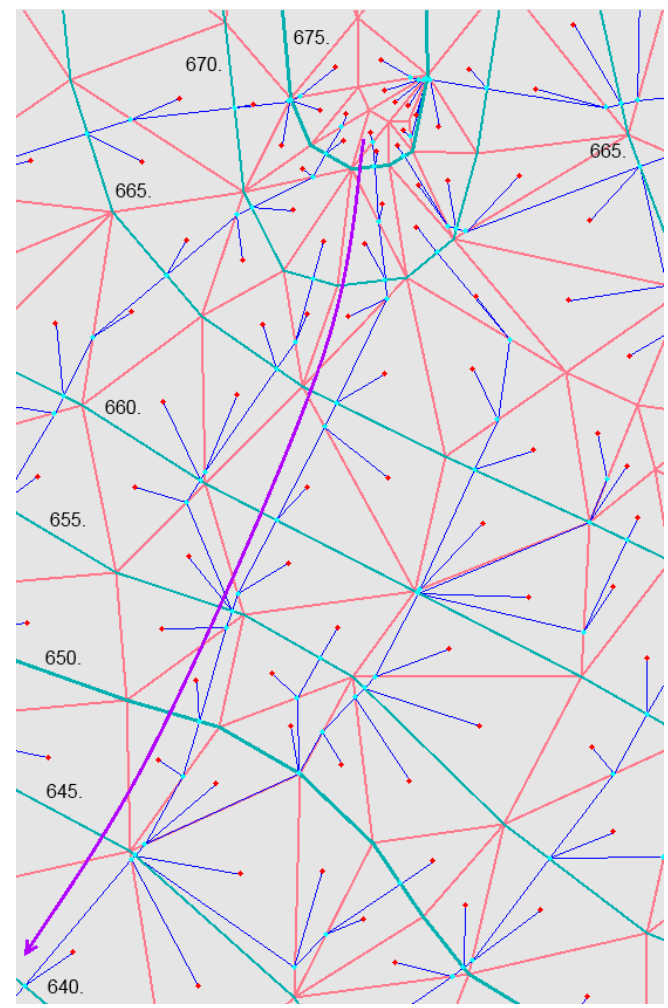

(a)

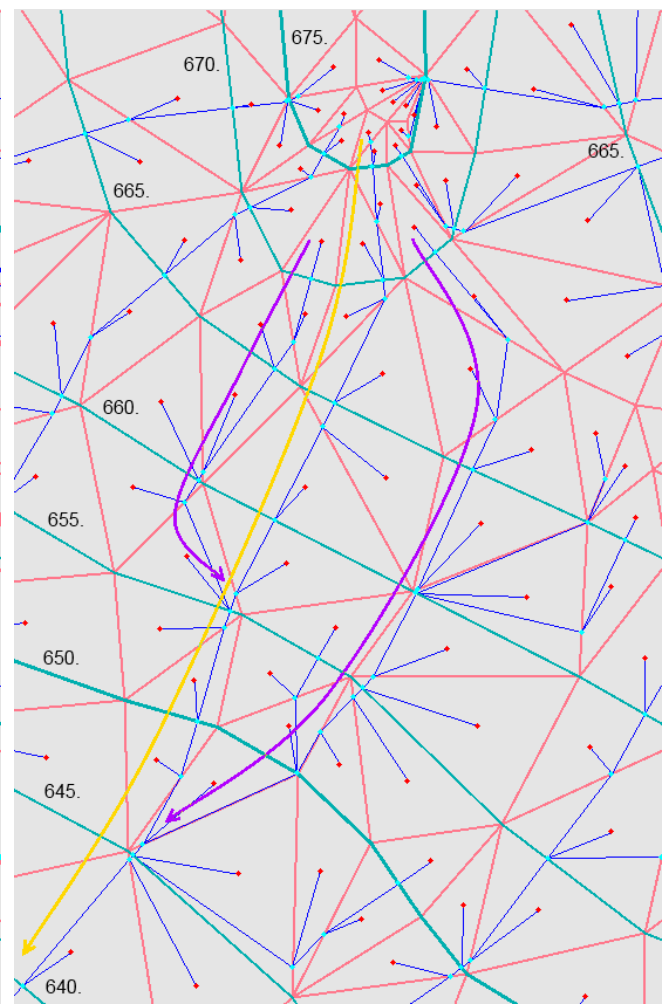

(b)

Figure 3.11 - Connection of drainage paths: (a) First drainage path (purple) initiated at a higher triangle centroid. (b) Subsequent drainage paths (purple) are connected to the first drainage path (orange). (continue) 


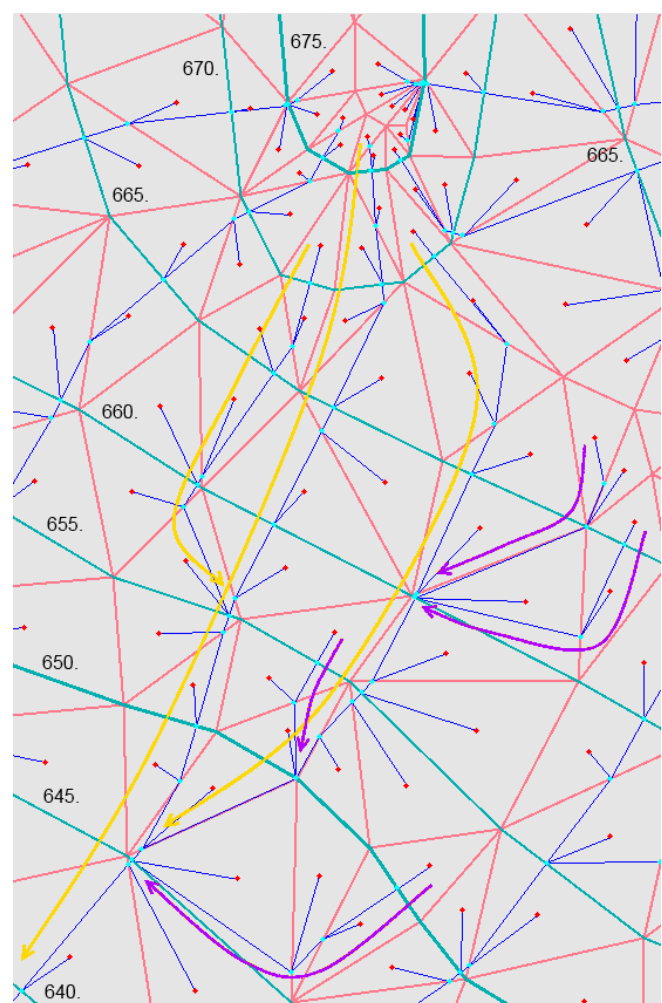

(c)

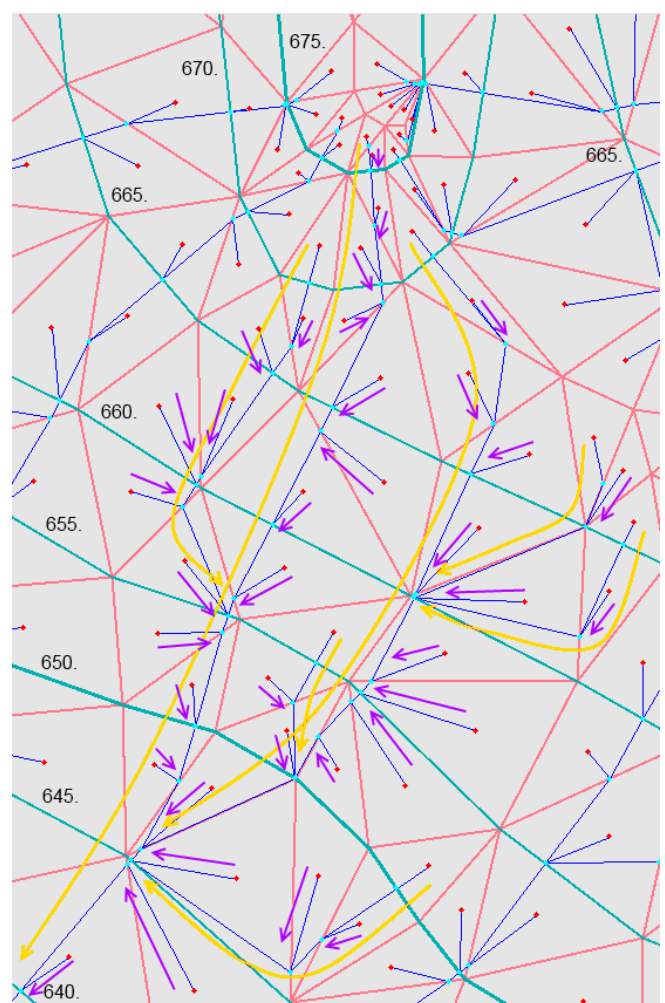

(d)

Figure 3.11 - Connection of drainage paths: (c) New drainage paths (purple) are also connected to the drainage paths already defined (orange). (d) Flow segments (purple) from triangles centroids are connected to the drainage paths (orange). (conclusion)

This drainage structure is an important contribution of this work and referred to as a drainage graph, which can derive potential drainage patterns since it allows the calculation of accumulated flows that are used to generate drainage networks from threshold values. The drainage graph is comprised of nodes and edges defined from the drainage paths as illustrated in figure 3.11, where the nodes are depicted by the dots in cyan and red, while the edges by the blue flow segments connecting pairs of nodes. All the nodes and edges are defined from the drainage paths as follows.

- Drainage Graph:

- Nodes:

a) Starting points (triangles centroids);

b) Intersections between gradient vectors and triangles edges (either along edges or at vertices); 
c) Vertices of channel edges.

- Edges:

a) Flow segments across triangles given by the gradient vectors (overland flow);

b) Flow segments along triangles edges (channel flow).

More formally, the drainage graph can be mathematically defined as $D G=(V, E)$, where $D G$ stands for Drainage Graph, $V$ is the set of all the points considered as the nodes and $E$ is the set of all the flow segments that define the edges. ${ }^{1}$

A relevant property of the drainage graph is that it defines a tree structure, more precisely, a directed acyclic graph. The flow segments always follow one direction and do not generate paths that reach nodes already processed, i.e., the drainage graph does not contain cycles between the nodes. This is a fundamental property for the calculation of the flows accumulated in each node of the drainage graph.

\subsubsection{Accumulated flows}

As already mentioned, the drainage graph defined from the drainage paths can be used for the calculation of the flows accumulated at specific points of the TIN surface. Additionally, these points comprise all the nodes of the drainage graph from which the flow segments, that represent the flow directions, promptly indicate the sources and destinations of the water flows accumulated from node to node.

More precisely, the values accumulated in the nodes of the drainage graph are defined from the areas of the triangles crossed by the flow segments. Therefore there are two different cases that can occur regarding how the triangles contribute to the flow accumulated in each node. Both cases are specified as follows:

- The flow segment crosses the interior of a triangle, so the contributing area is defined by the area of the triangle;

- The flow segment follows along a channel edge between two triangles. In this case, the contributing area is defined by the sum of the areas of both triangles adjacent by the channel edge.

\footnotetext{
${ }^{1}$ The nodes of a graph are commonly considered as vertices, so $V$ is usually used as the notation to represent the set of nodes, although this work refers to the vertices of the graph as nodes and makes use of the $V$ notation.
} 
In the former case, the value accumulated in the destination node is the value accumulated in the source node plus the area of the triangle crossed by the flow segment. In the latter case, both triangles adjacent by their common edge, which comprises a channel edge, contribute to the flow accumulated in the destination node, so that the total value to be accumulated is also defined by the value accumulated in the source node but now added with the areas of the two adjoining triangles.

Figure 3.12 illustrates the two cases of the contributing areas assigned to the flows accumulated in the nodes of the drainage graph, where a flow segment either crosses a triangle (overland flow) or follows along a channel edge (channel flow). Nodes in red are triangles centroids ${ }^{2}$, while nodes in cyan are the intersection points calculated when tracing the drainage paths.

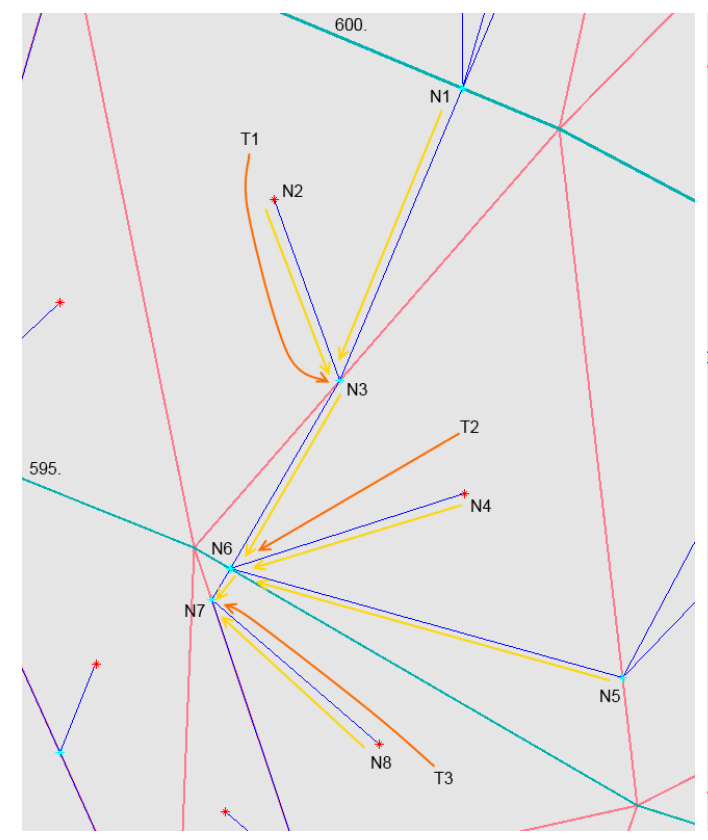

(a)

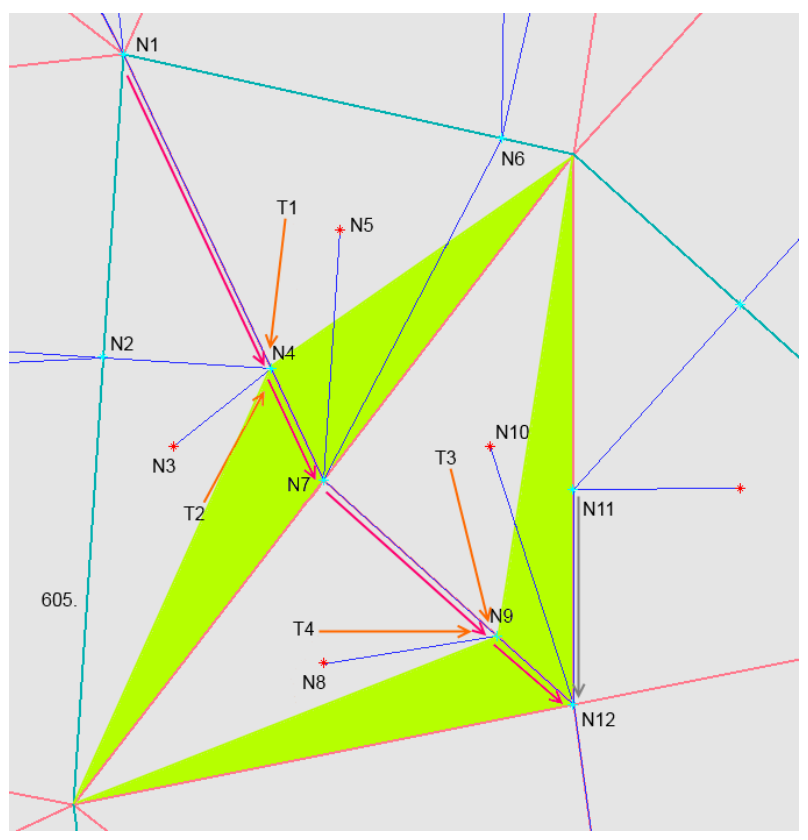

(b)

Figure 3.12 - Contributing areas from triangles in two different cases: (a) contributing area from triangles crossed by flow segments. (b) contributing areas from triangles adjacent by a channel edge.

Overland flows are depicted in the example of figure 3.12(a). The nodes $N_{1}$ and $N_{2}$ contribute to the flow accumulated in node $N_{3}$ (yellow arrows). In addition, as the triangle $T_{1}$ is crossed by the flow segments $N_{1} N_{3}$ and $N_{2} N_{3}$, its area is also accumulated in node $N_{3}$ (orange arrow). It is worthy mentioning that although two

\footnotetext{
${ }^{2}$ Each triangle centroid is assigned an accumulated flow equals to zero.
} 
flow segments cross the triangle $T_{1}$, its area is accumulated only once. Similarly, this procedure of accumulated flows is also valid for the other nodes and triangles shown in the figure.

The other example exhibited in figure 3.12(b) depicts the case of channel flows and how the contributing areas are accumulated in each node. In this example, besides the presence of flow segments that define overland flows (segments $N_{2} N_{4}, N_{3} N_{4}$, $N_{5} N_{7}, N_{6} N_{7}, N_{8} N_{9}$ and $N_{10} N_{12}$ ), there also exist flow segments along channel edges, which are shown in light red (segments $N_{1} N_{4}, N_{4} N_{7}, N_{7} N_{9}$ and $N_{9} N_{12}$ ).

The flow segment $N_{1} N_{4}$ determines that, besides the node $N_{1}$, both the triangles $T_{1}$ and $T_{2}$ contribute to the flow accumulated in node $N_{4}$ (orange arrows). Moreover, nodes $N_{2}$ and $N_{3}$ also contribute to the flow accumulated in node $N_{4}$ since the flow segments $N_{2} N_{4}$ and $N_{3} N_{4}$ define overland flows over the triangle $T_{2}$. However, despite the channel flow segment $N_{1} N_{4}$ be incident to triangle $T_{2}$ and the triangle $T_{2}$ be crossed by two overland flow segments, its area is accumulated only once in node $N_{4}$. The same procedure is performed on node $N_{9}$ that also belongs to a channel flow segment.

Additionally, even though the nodes $N_{7}$ and $N_{12}$ are also located at channel edges, these nodes have accumulated flows calculated in a different way because they are vertices of triangles which are not located somewhere along the edge, so that the contributing areas accumulated from their upstream nodes, i.e., nodes $N_{4}$ and $N_{9}$, respectively, are defined by a fraction of the total areas from both triangles adjacent by the edge. This specific calculation avoids these areas to be accumulated twice from node to node in the sequence of flows along channel edges. Figure 3.12(b) illustrates the contributing areas as a fraction of each triangle depicted by the green regions inside the triangles.

It is also important to notice in figure $3.12(\mathrm{~b})$ that the flow segment $N_{11} N_{12}$, indicated by the gray arrow, is not defined by a channel edge. This flow segment was defined when the drainage path coming from the right reached the node $N_{11}$ and encountered the other drainage path that previously reached the node $N_{12}$, so that the drainage path from $N_{11}$ was just connected to the other drainage path at the node $N_{12}$, which is the outflow node of the triangle $T_{3}$.

Still regarding the drainage graph, the sources and destinations of water flows over the TIN surface are represented by the endpoints of each flow segment, i.e., by the nodes connected through the edges of the drainage graph. More specifically, each 
node $p$ of the drainage graph can have zero or more source nodes referenced as the upstream nodes, which are the inflow nodes that contribute to the water flow accumulated in the node $p$.

In addition, each node $p$ has also only one destination node referenced as the downstream node, which is the outflow node where the water flow accumulates from the node $p$. Figures 3.13(a) and 3.13(b) give examples of the upstream and downstream nodes connected to specific nodes of the drainage graph.

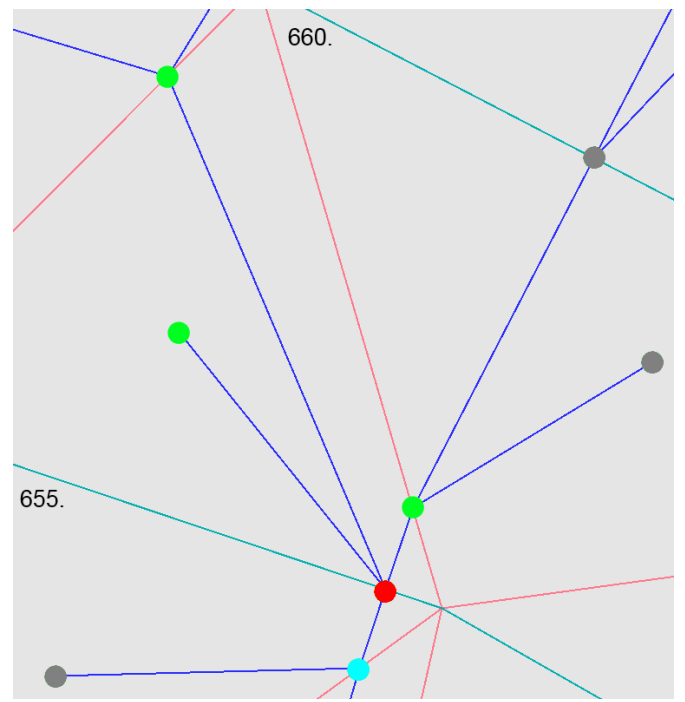

(a)

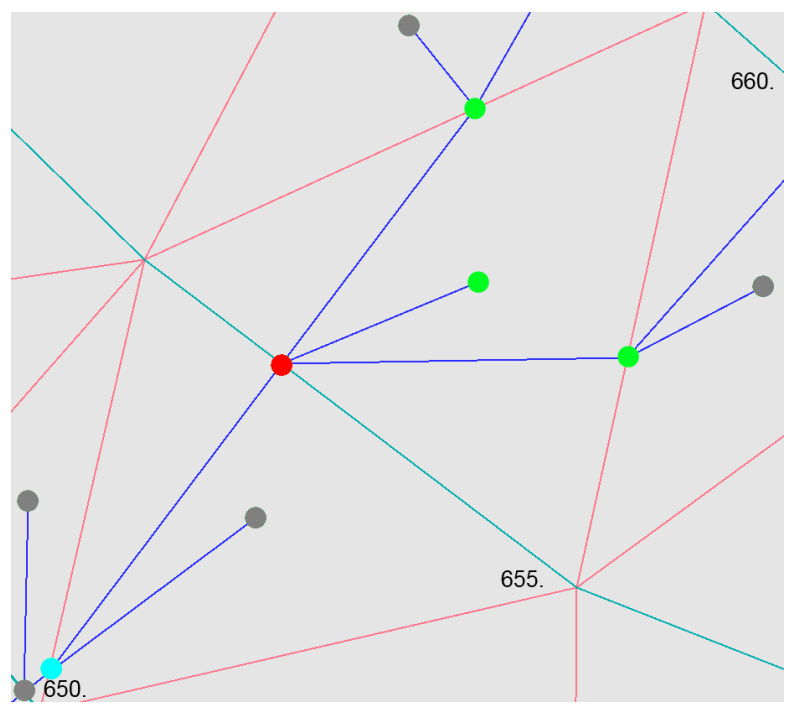

(b)

Figure 3.13 - Nodes of the drainage graph in red with their upstream nodes in green and downstream nodes in cyan

Considering the entire drainage graph structure, the water flows accumulated in each node are calculated by performing a specific procedure on the drainage graph that process the nodes according to a particular ordering. As mentioned before, the drainage graph is defined by a directed acyclic graph that determines one-direction flows between points on the TIN surface. For this reason, before calculating the accumulated flows, it is therefore necessary to determine an ordering of the nodes that allows a correct evaluation of each flow, so that the directed acyclic graph is the right structure for this purpose.

Directed acyclic graphs, or DAG for short, are used in applications where there exist precedences among events. Any DAG presents a specific ordering of the vertices such that if a DAG $G=(V, E)$ contains an edge $(u, v)$, then $u$ appears before $v$ in the 
ordering (CORMEN et al., 2001). This ordering is called a topological sort of the $\mathrm{DAG}$ and it is performed by a procedure beginning at the nodes of in-degree zero.

In the case of the drainage graph, the procedure begins at the nodes without upstream nodes. Next, whenever a node $p$ has each of its upstream nodes already processed, i.e., all the flows from the upstream nodes are accumulated in the node $p$, then the node $p$ can also be used for the calculation of the accumulated flows and this procedure continues until all the nodes have been processed.

Figure 3.14 gives an example of how the flows are accumulated in some nodes of the drainage graph by following a topological sort of its nodes. In this example, the flows are accumulated until reaching the node at the bottom (green). The steps taken in the procedure are as follows:

a) Initially, only triangles centroids (red) have zero upstream nodes, so the flows are accumulated from the centroids in their respective downstream nodes (orange arrows);

b) Next, all the upstream nodes of the nodes $N_{1}, N_{5}$ and $N_{8}$ have already been processed (yellow arrows) and the flows are accumulated in nodes $N_{2}, N_{6}$ and $N_{10}$, respectively;

c) The same happens now to nodes $N_{2}, N_{6}$ and $N_{10}$. In this case, the flows are accumulated in nodes $N_{3}, N_{7}$ and $N_{9}$, in this order;

d) At this point, only node $N_{3}$ in the sequence has its upstream nodes already processed (yellow arrows), so the flow accumulates from node $N_{3}$ to its downstream node, in this case, node $N_{4}$;

e) Next, the flow from node $N_{4}$ accumulates in node $N_{7}$;

f) Similarly, the flow from node $N_{7}$ accumulates in node $N_{9}$;

g) One more step and the flow from node $N_{9}$ accumulates in node $N_{11}$;

h) Finally, the flow from node $N_{11}$ accumulates in node $N_{12}$ (green). 


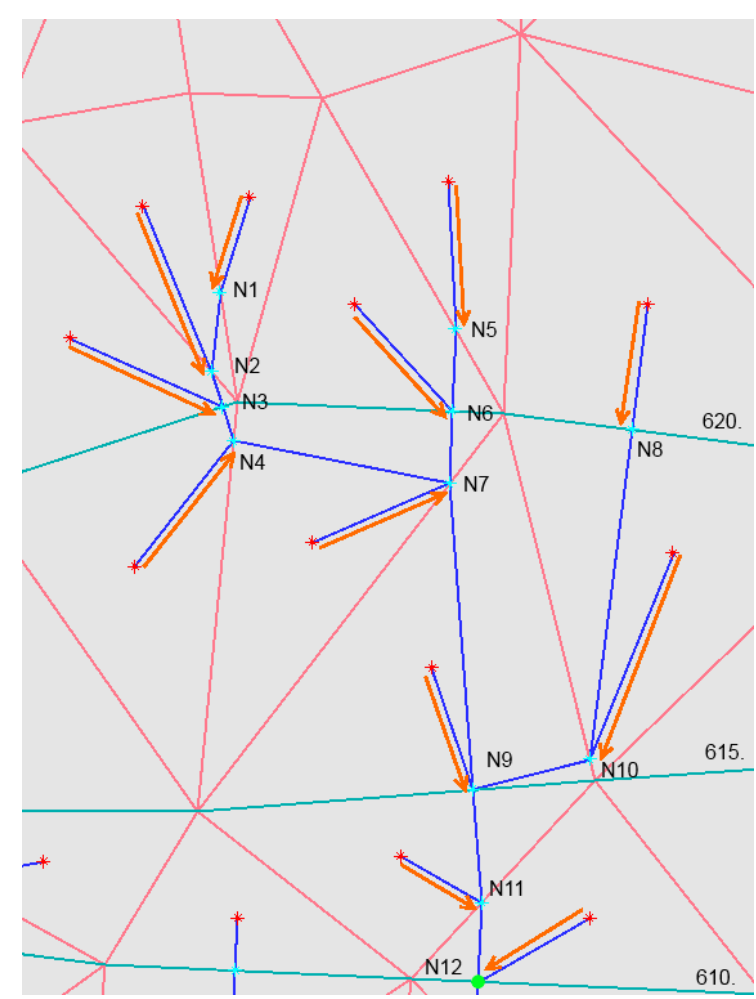

(a)

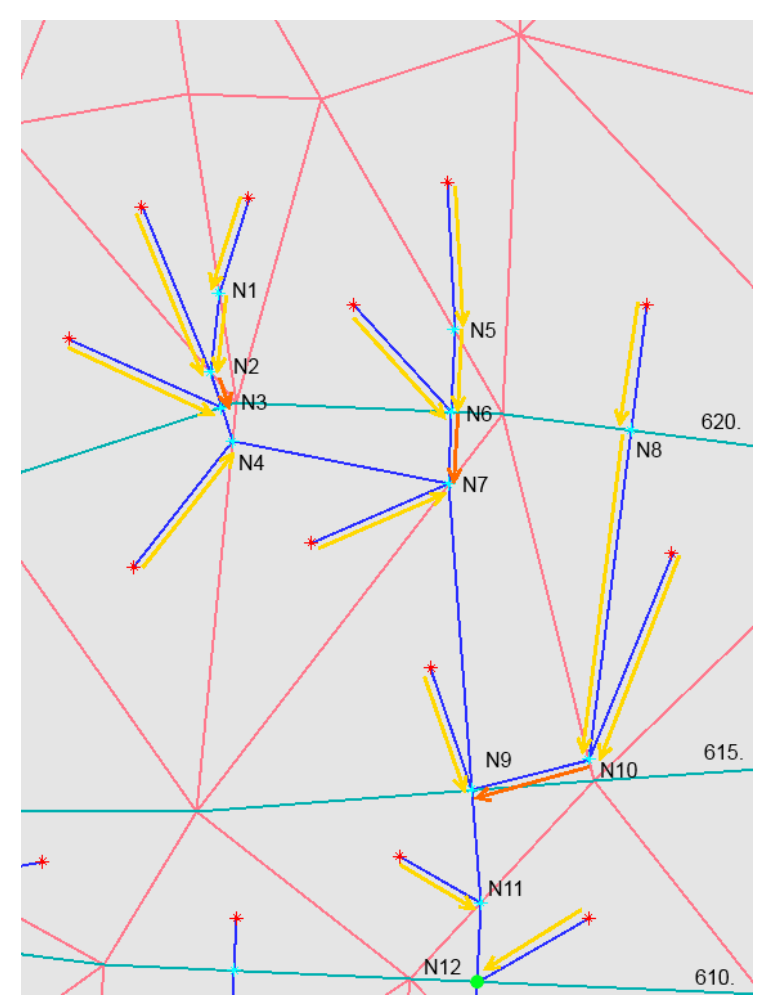

(c)

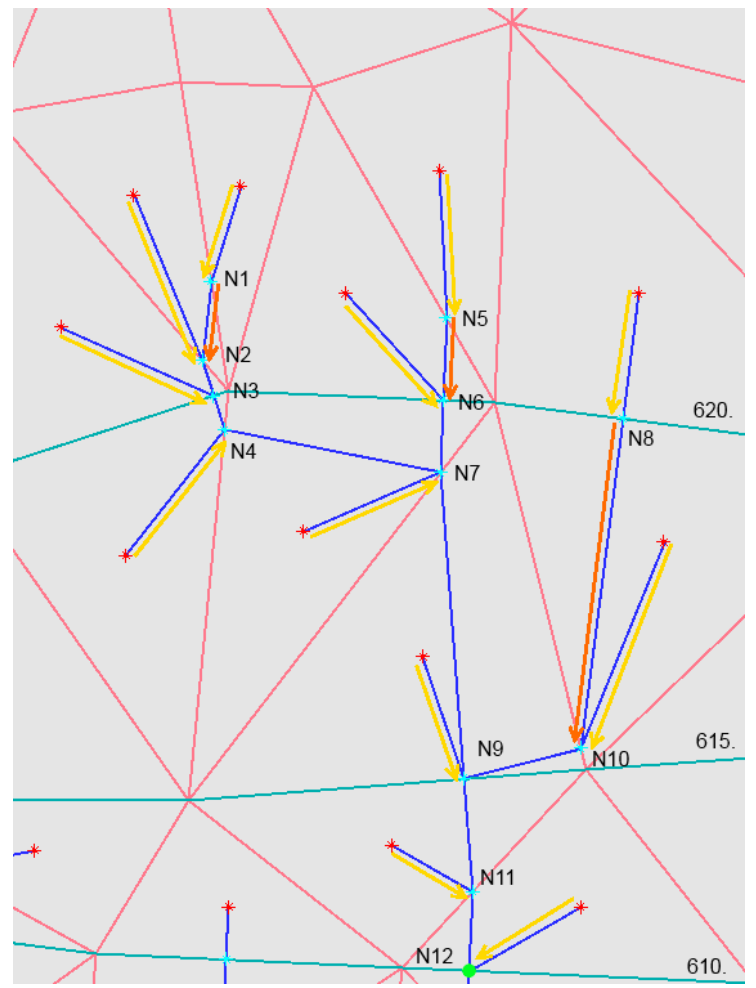

(b)

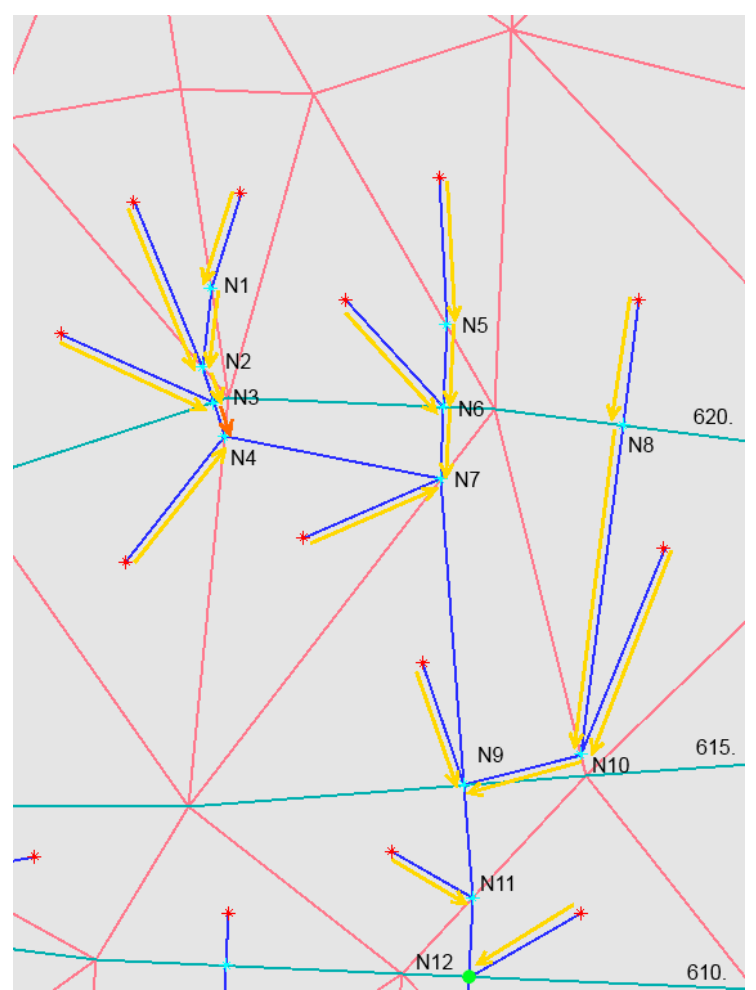

(d)

Figure 3.14 - Accumulated flows defined by a topological sort of the nodes of the drainage graph (continue) 


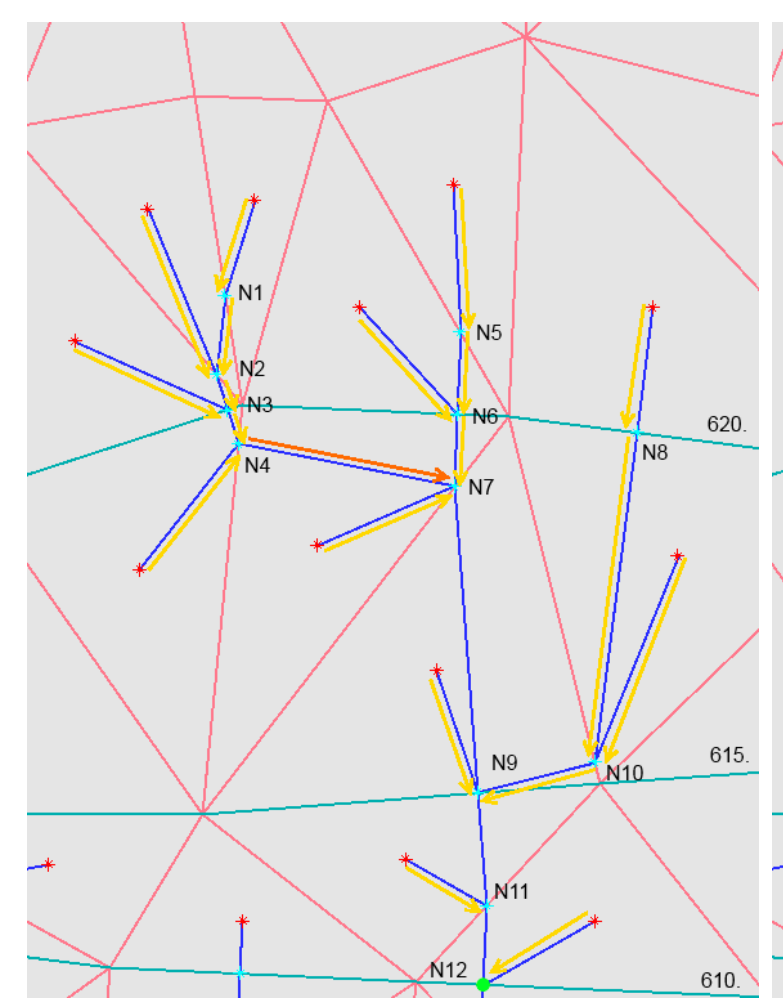

(e)

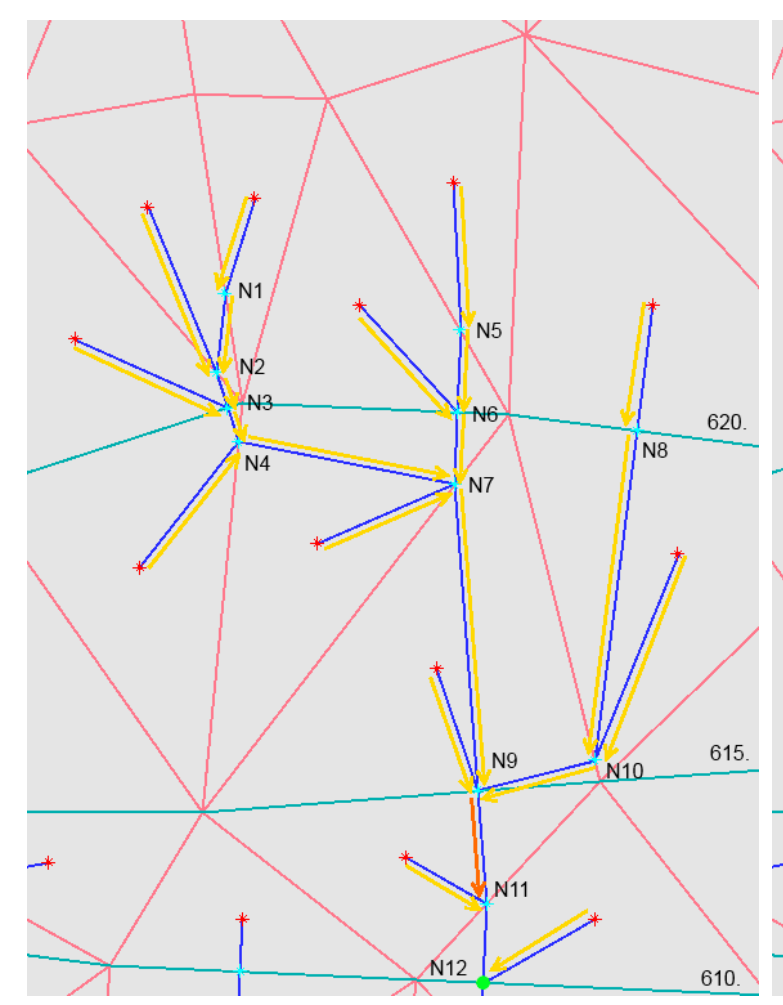

(g)

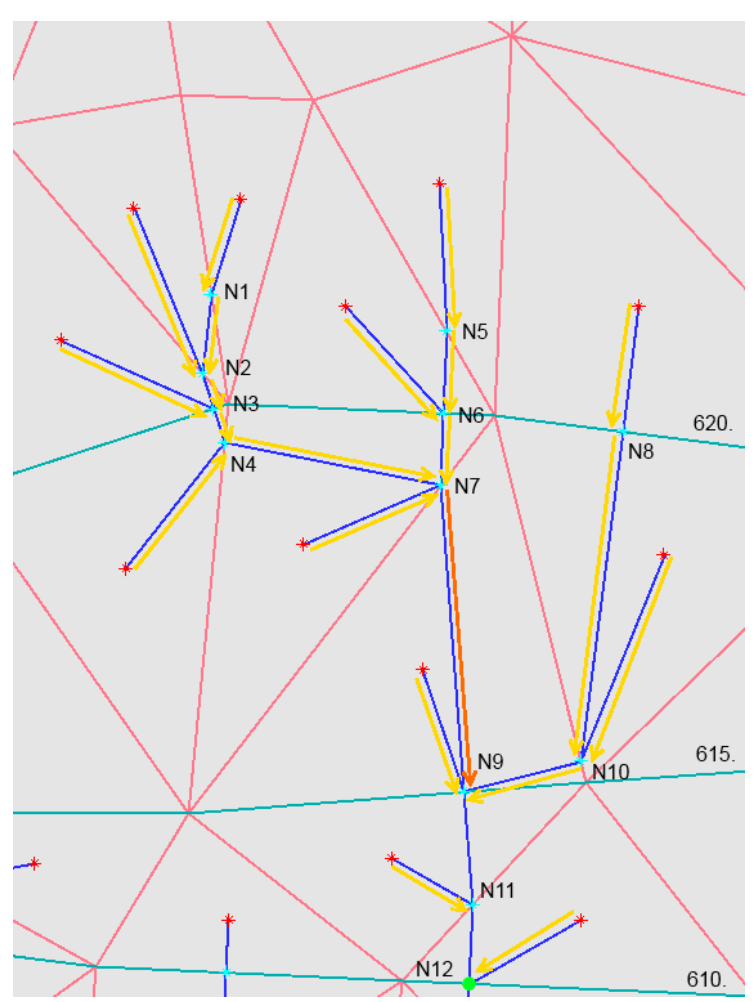

(f)

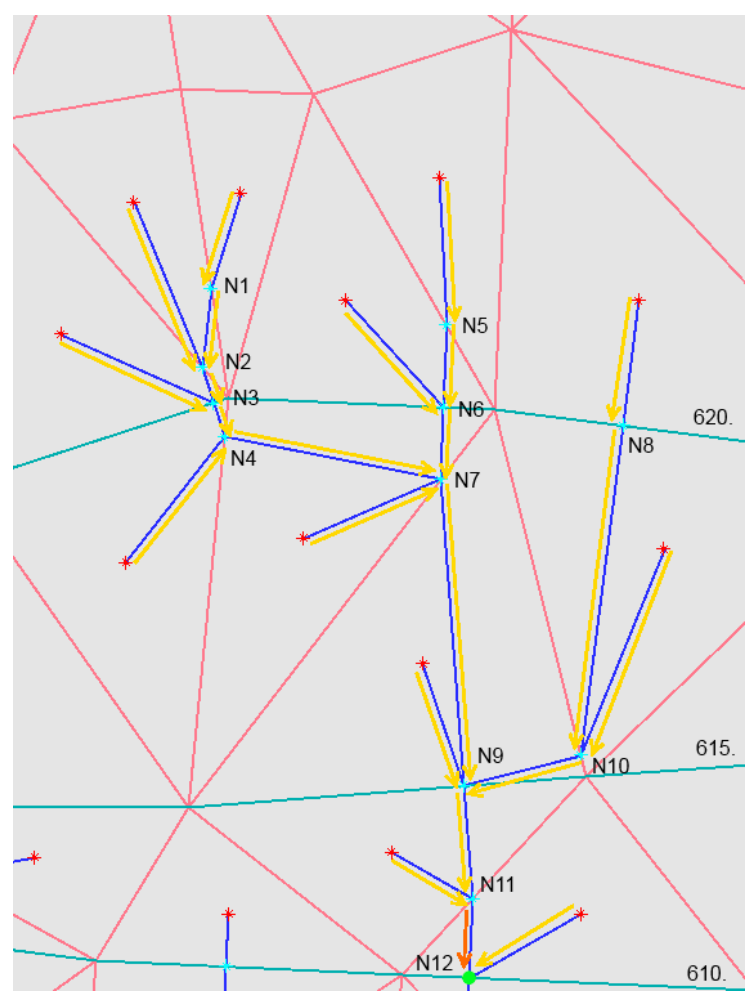

(h)

Figure 3.14 - Conclusion 


\subsubsection{Drainage networks}

Drainage networks constitute one of the most important hydrological structures with useful applications in hydrological models and resource management plans (O'CALLAGHAN; MARK, 1984) since a drainage network provides significant information on water resources, possible flood areas, erosion and other natural processes (AGARWAL et al., 1996). This structure is able to represent the main courses of water over a terrain surface and it is also an essential component for delineating the watersheds of a given region.

Some previous works that analyze the extraction of drainage structures from TIN terrain models define drainage networks by either detecting only the channel edges present in the TIN (JONES et al., 1990), therefore restricting the number of flow segments that comprise the drainage network, or by also including flow segments that strictly follow the paths of steepest descent in each triangle (GUERCIO; SOCCODATO, 1996; YU et al., 1996; TSIROGIANNIS, 2011), which represents a better approximation of the flow patterns. However, none of these works provide specific procedures for the generation of drainage networks from accumulated flows over the TIN model.

In this work, drainage networks are generated based on the accumulated flows calculated from the drainage graph, both described in previous sections. The drainage networks are defined from specific threshold values compared to the flow accumulated in each node of the drainage graph, then subsequently by including all the flow segments that leave nodes which have accumulated values equal to or greater than the given threshold.

Figure 3.15 presents a small region with different cases of flow segments that are included in the drainage network depending on the flow values accumulated in each node of the drainage graph, which are then compared to specific threshold values in each case. The accumulated flows of each node (cyan) as well as the area of each triangle (next to the centroids in red), both in $\mathrm{m}^{2}$, are also indicated in the figure.

Moreover, the flow segments included in the drainage networks are given in dark blue, while the ones not included are in light blue. Some of the flow segments located outside the larger contour line are colored in orange in all the figures, except figure 3.15(a), because the accumulated flows from their source nodes are not necessary in this example. 


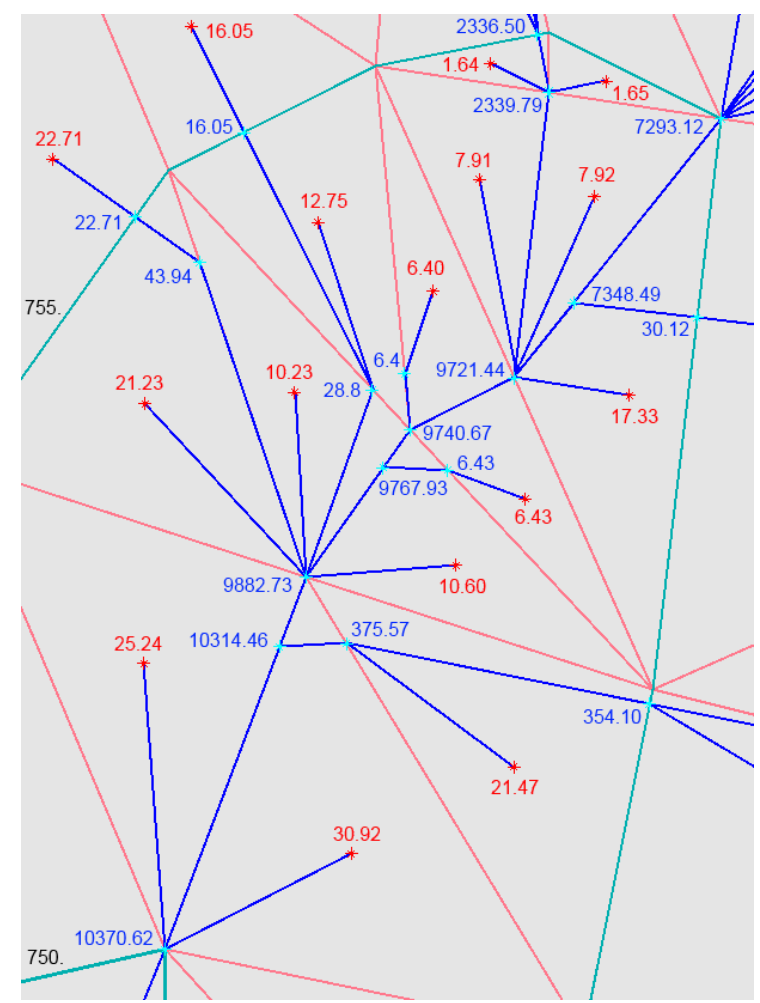

(a)

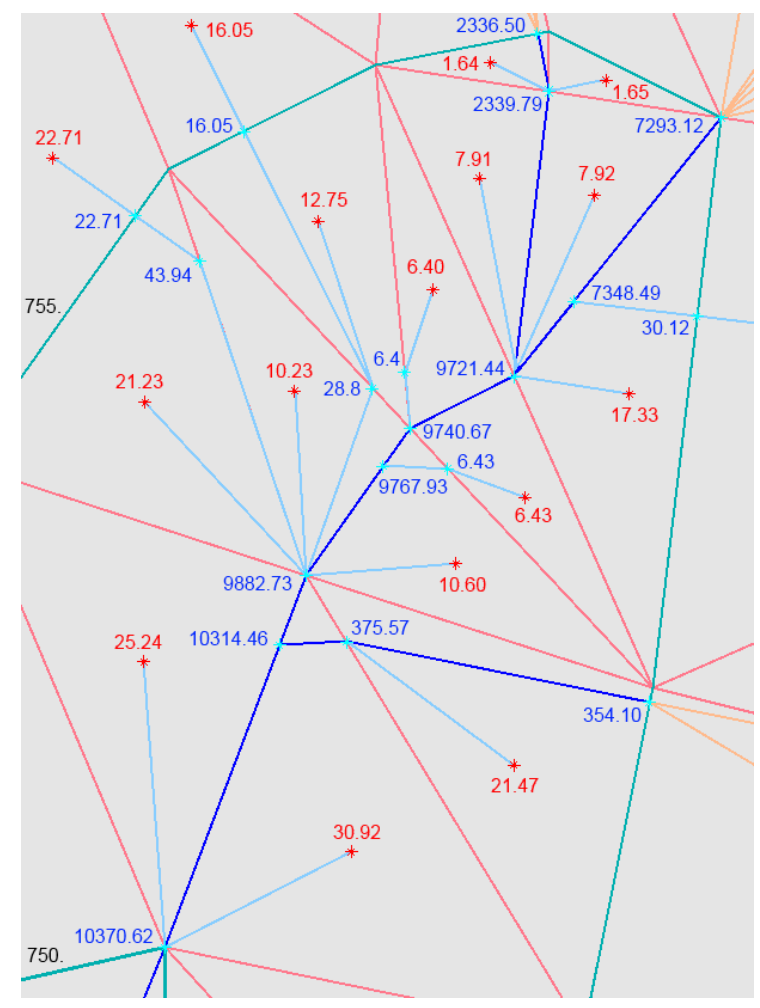

(c)

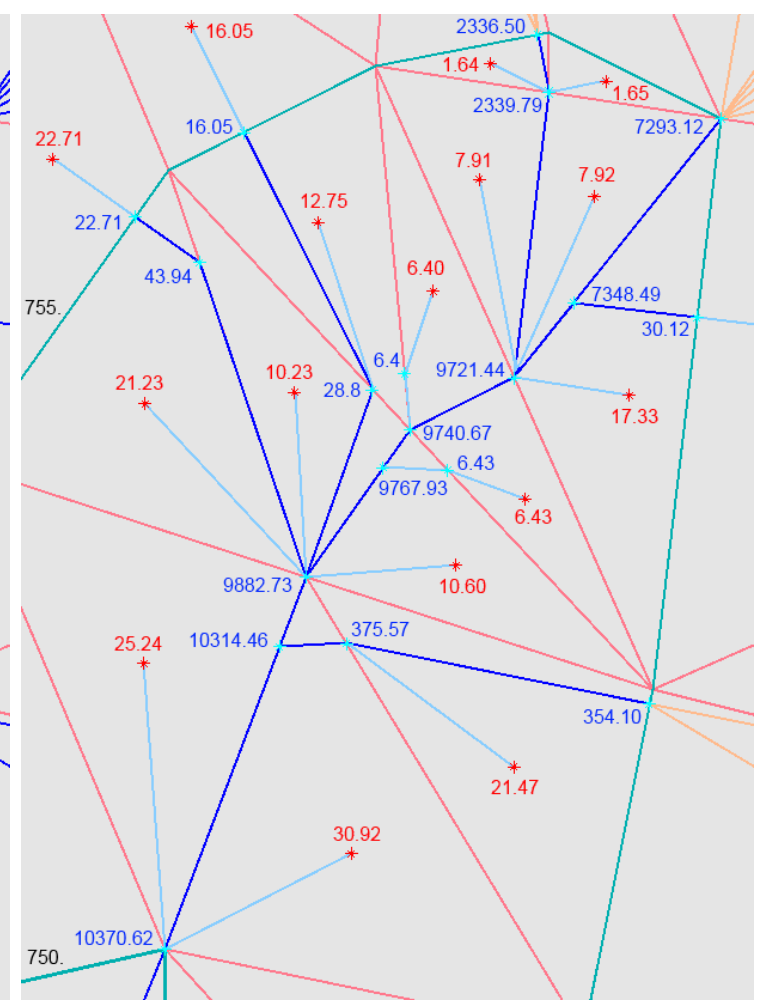

(b)

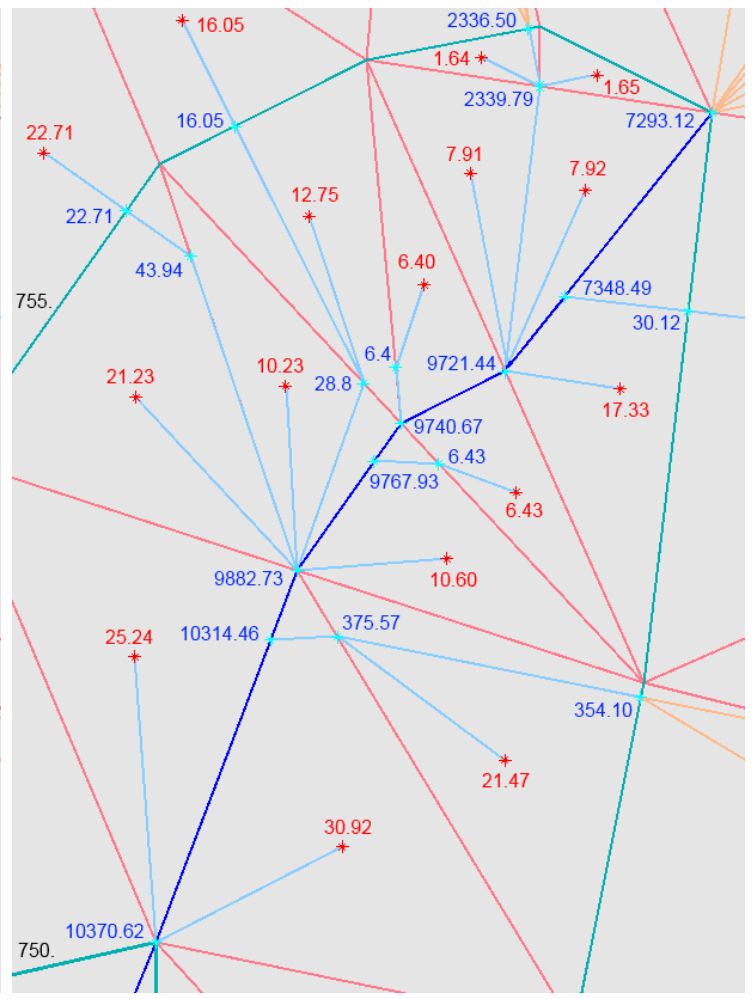

(d)

Figure 3.15 - Flow segments included in a drainage network depend on different threshold values $T$ : (a) $T=0$. (b) $T=10$. (c) $T=300$. (d) $T=7000$. (continue) 


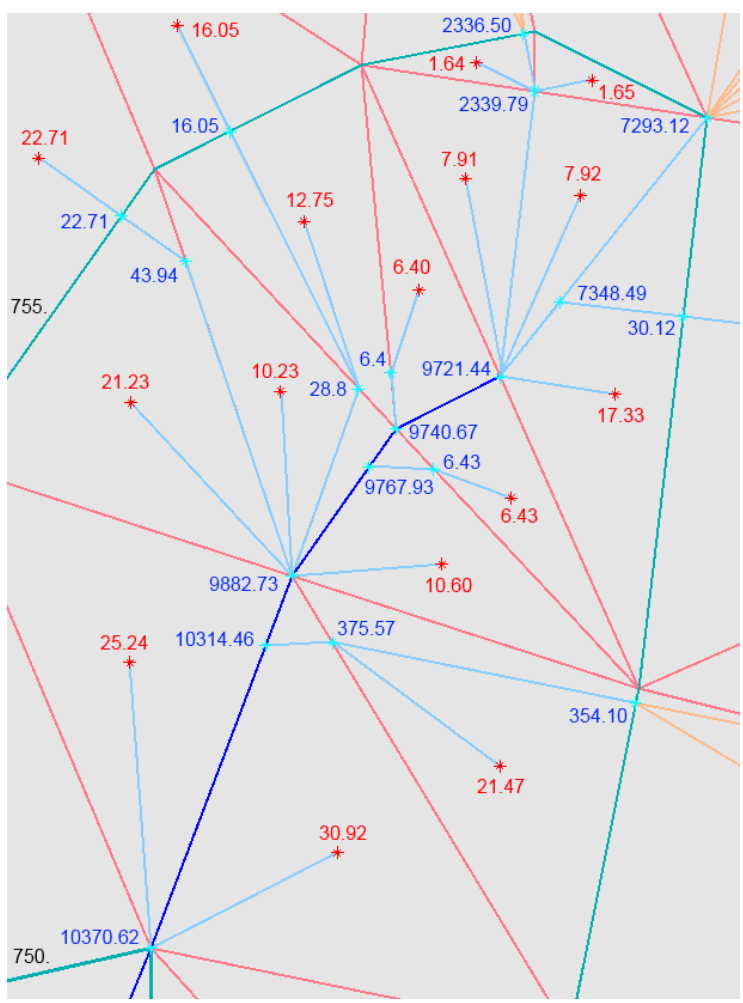

(e)

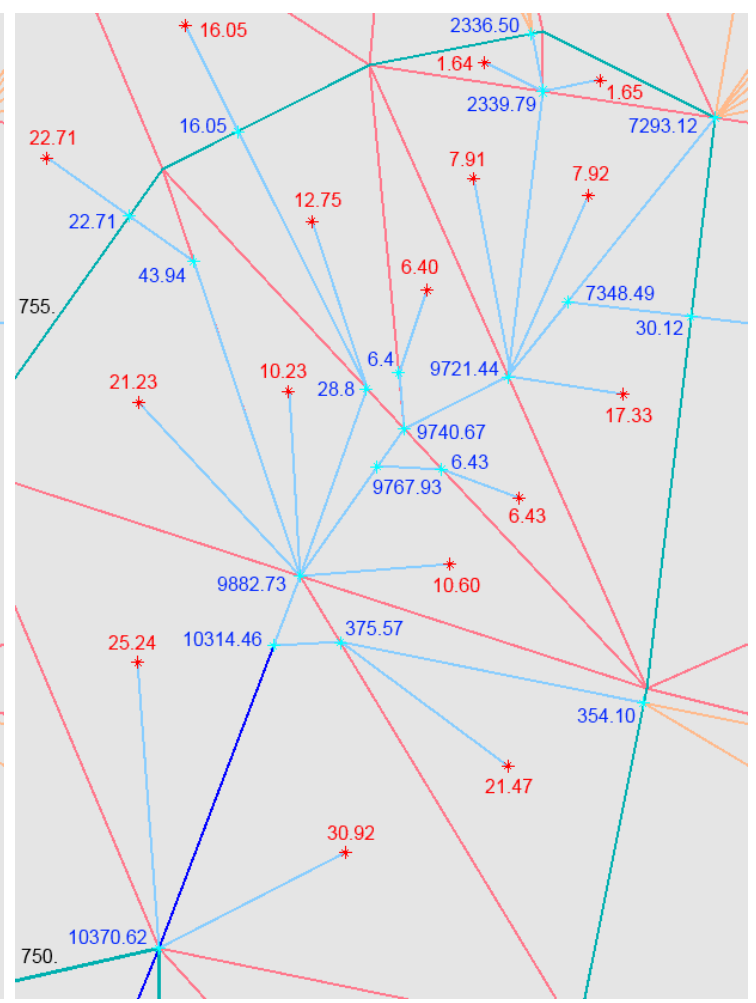

(f)

Figure 3.15 - Flow segments included in a drainage network depend on different threshold values $T$ : (e) $T=9000$. (f) $T=10000$. (conclusion)

It is possible to notice from this example that the distance in the $x y$ plane between the nodes of the drainage graph does not indicate whether a flow segment is included or not in the drainage network. Therefore the main courses of water are defined only from the flow segments that contain nodes with high values of accumulated flows relative to a given threshold, even though there exist many flow segments located close to the main water course. The point here is that the longest the drainage path, then more likely it is part of the main courses of water since the accumulated flow values increase from node to node along the path.

As another example, figure 3.16 exhibits distinct drainage networks generated from a larger region. These drainage networks also differ one from another due to the specific threshold values chosen in each case. It can be noticed that when the threshold value is low, then more flow segments are included, resulting in a denser drainage network. On the contrary, when the threshold value is high, then the number of flow segments included in the drainage network is reduced, thus only remaining the main courses of water over the terrain surface. 


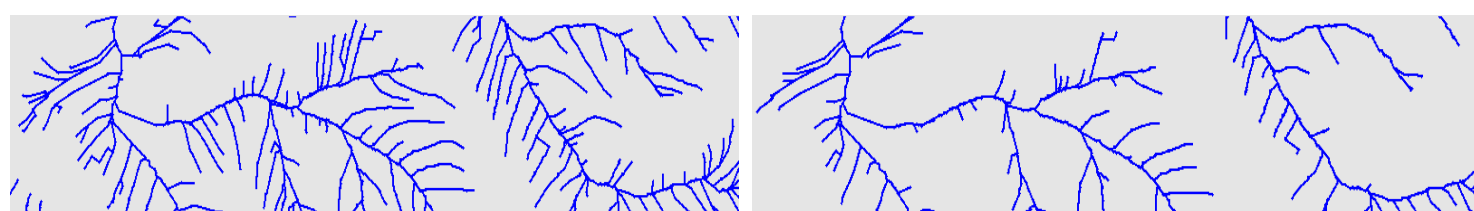
- M I ha

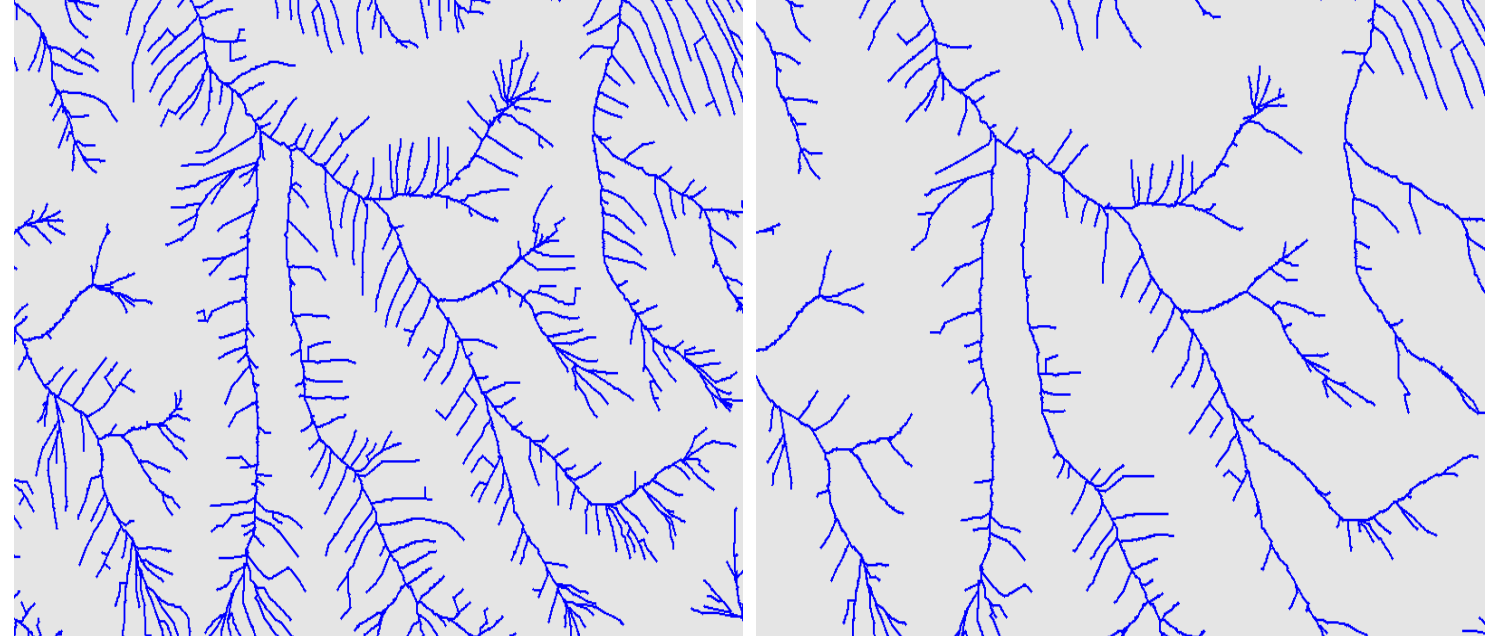

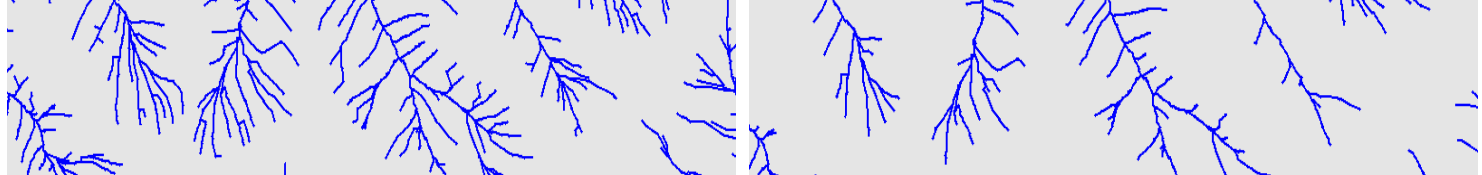

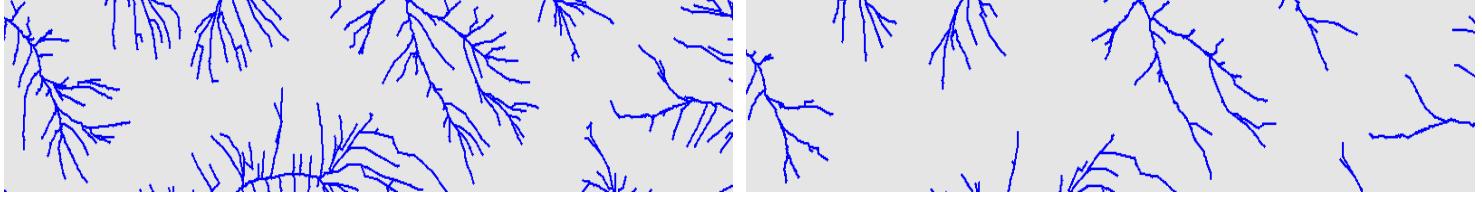

(a)

(b)

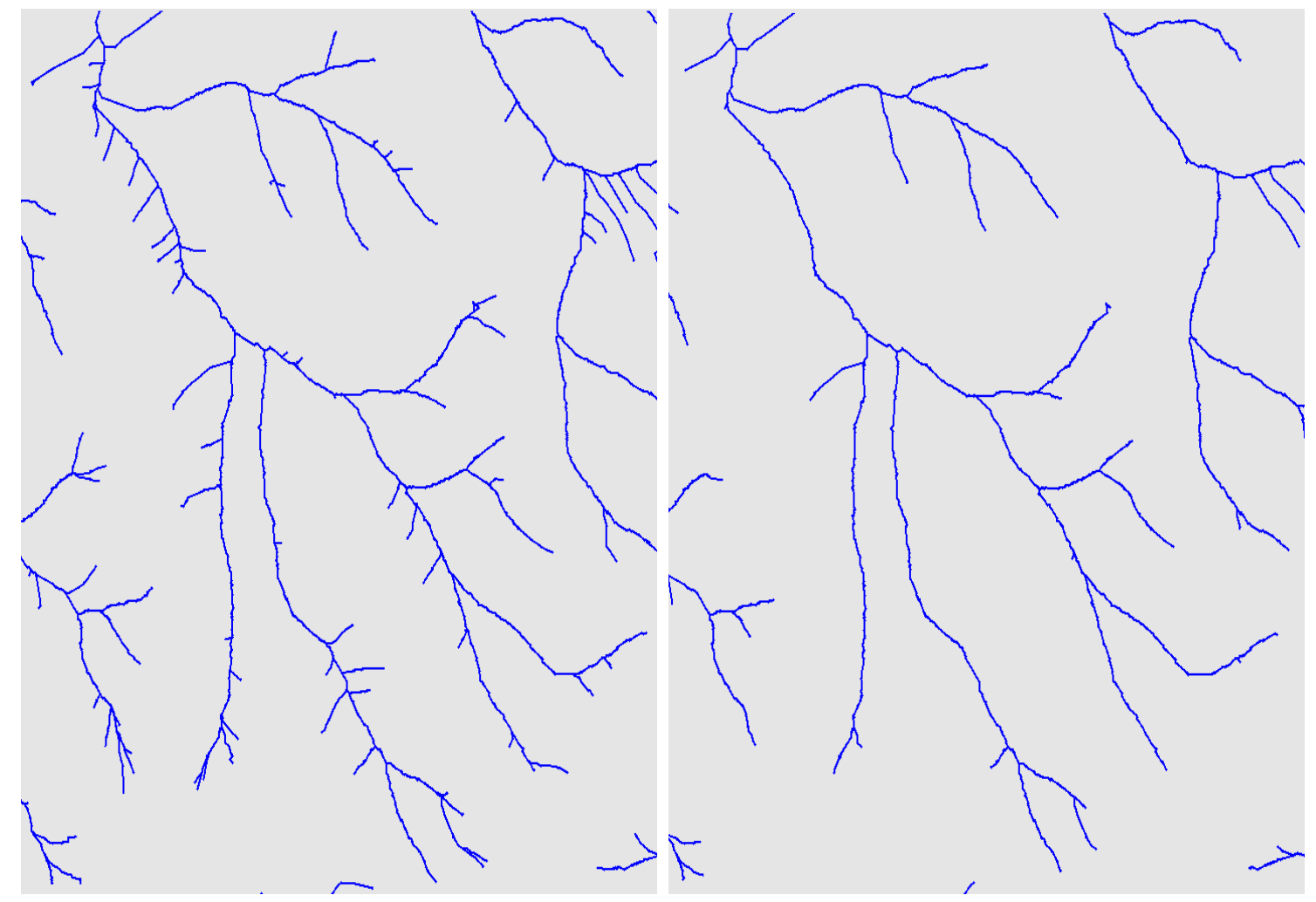

(c)

(d)

Figure 3.16 - Drainage networks generated from different threshold values $T$ : (a) $T=1000$. (b) $T=2000$. (c) $T=4000$. (d) $T=8000$. 


\subsubsection{Watersheds and subwatersheds delineation}

Watersheds or drainage basins determine a significant concept in hydrology since a watershed may be defined as the area that contributes water to a particular region of a drainage network. In essence, it forms a natural unit for hydrological data as the total quantity of water that flows out of the watershed can be measured. Thus the delineation of watersheds plays an important role in hydrological studies with a wide variety of applications (ZHANG et al., 1990).

The watersheds of a given region are represented by distinct and disjoint areas which are determined in accordance with the water flow patterns present on the terrain surface of the region. More precisely, watersheds can be defined by polygons on the terrain model (in the case of this work, on the TIN) where all the interior points drain water to specific flow segments of the drainage network generated from the region. Therefore each flow segment of a drainage network is related to particular points on the terrain surface from which the outflows contribute to the flow of water that reaches the flow segment.

In this work, watersheds are delineated from the flow segments that constitute the drainage graph structure previously described, which strictly follows the paths of steepest descent, so that in this case the delineation represents a more realistic approximation (GUERCIO; SOCCODATO, 1996; YU et al., 1996; TSIROGIANNIS, 2011) in comparison to a delineation that considers only the channel edges of the TIN (JONES et al., 1990).

It is worthy mentioning here that since each node of the drainage graph contains references to the downstream and upstream nodes, then the upstream nodes references can be used to delineate the watersheds. Thus, in the TIN terrain model, the watersheds are automatically delineated from any point located in the drainage network, considered as an initial point, by always following the flow segments connected to each upstream node reachable from the initial point.

From this procedure, all the upstream nodes are recursively traversed along the drainage paths of the drainage graph, where each flow segment defined from a node to its upstream node then adds as part of the watershed either one triangle or even two triangles, in the case that the flow segment is located on a channel edge. This search procedure is performed until all the nodes with no upstream nodes have been reached. 
Figure 3.17 exhibits the flow segments of the drainage graph that are located in the drainage network (dark blue) and also the ones that are not in it (light blue) but that contribute to the flows accumulated in all the nodes present in the drainage network. In this example, the drainage network includes the nodes of the drainage graph which have accumulated values equal to or greater than a threshold value of $8000 \mathrm{~m}^{2}$. Figure 3.17(a) contains each triangle of the TIN crossed by the flow segments that delineate the watershed illustrated in figure 3.17(b) from the initial node at the bottom (cyan).

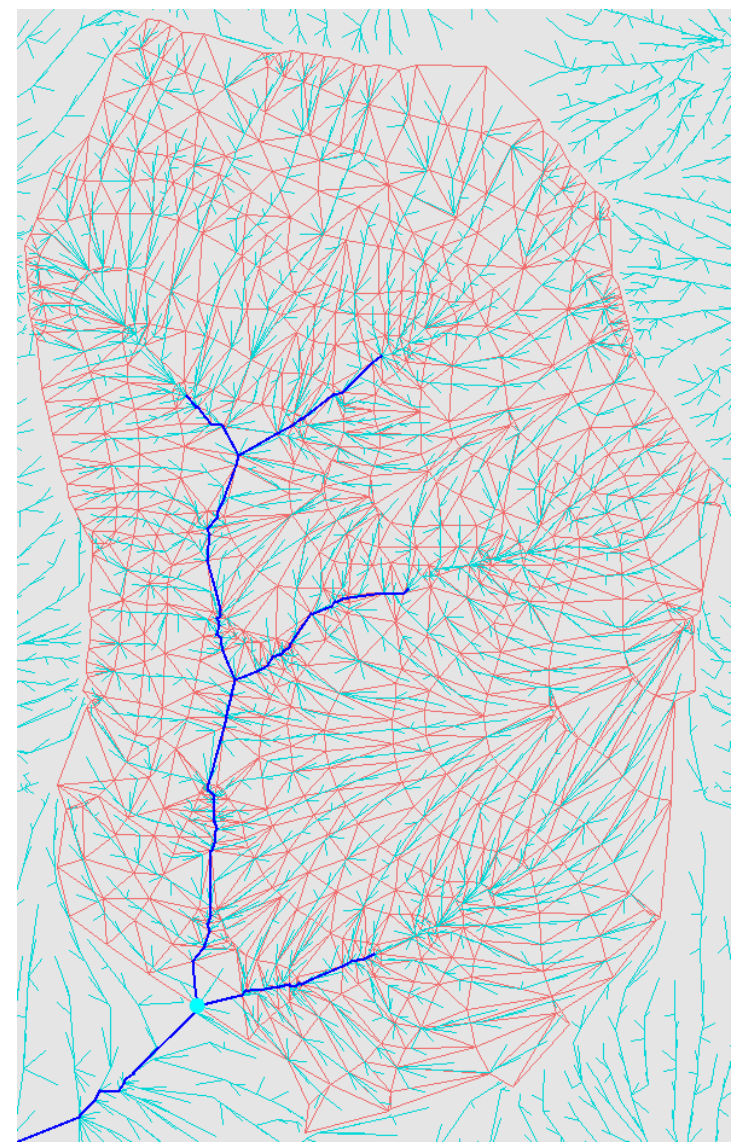

(a)

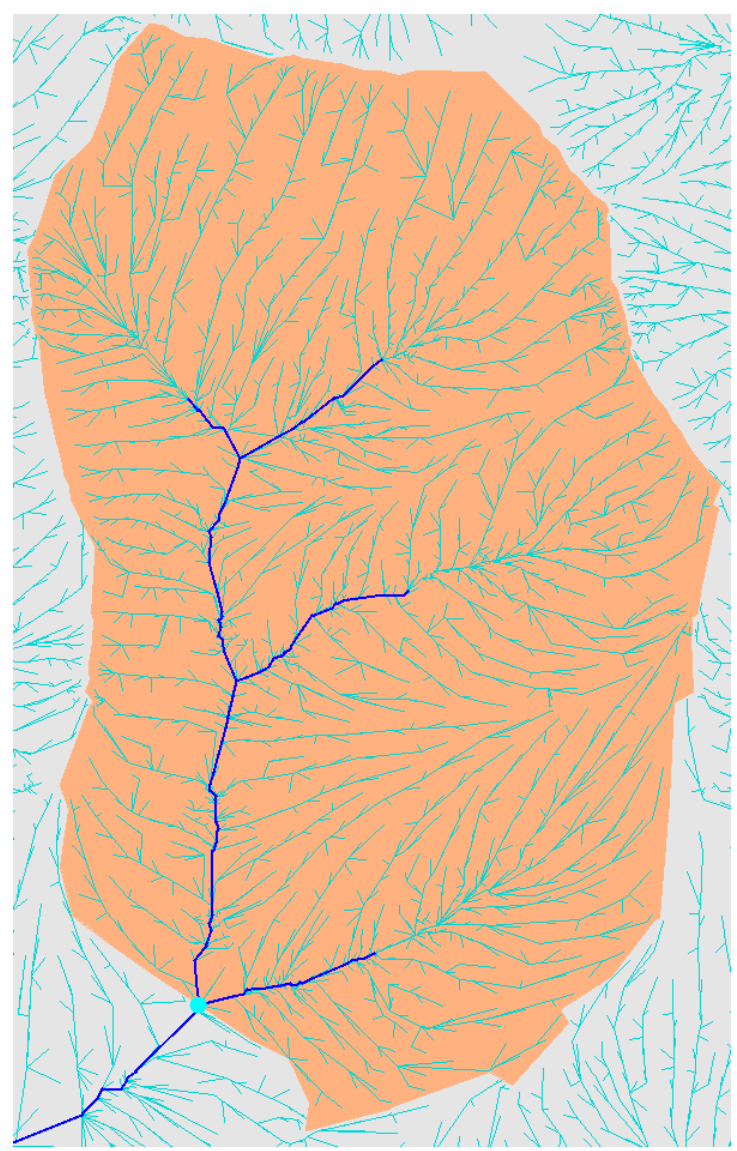

(b)

Figure 3.17 - Watershed delineated from the flow segments of the drainage graph: (a) triangles crossed by the flow segments of a specific region. (b) watershed delineated from the flow segments.

Besides the delineation of a single watershed by following all the upstream nodes of the flow segments from an initial point in the drainage network, it is also possible to subdivide a given watershed into several subwatersheds. This subdivision depends on the existent drainage segments that comprise the drainage network, i.e., parts of 
the drainage network where the flow segments follow a single path. In addition, the points where the drainage network presents branches determine distinct drainage segments, so that each drainage segment has one specific subwatershed associated. Figure 3.18 shows several drainage segments from part of a drainage network.

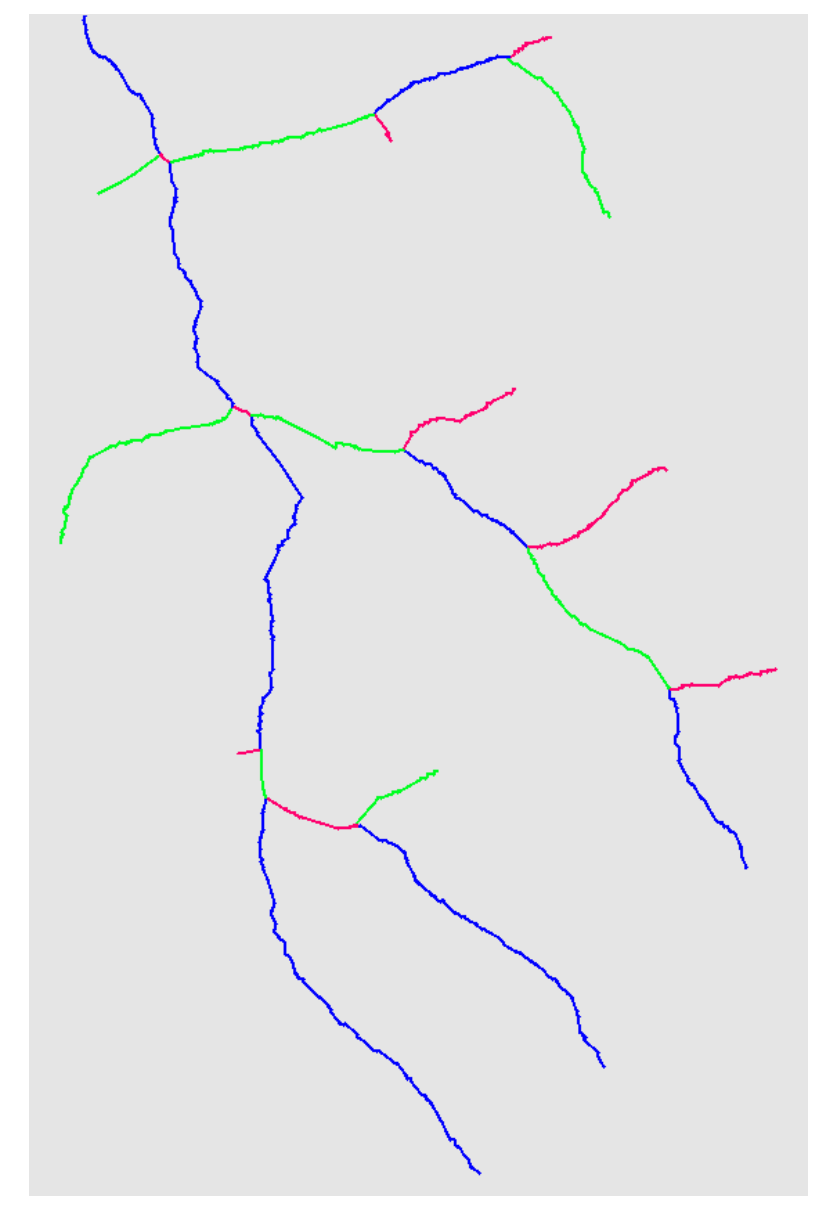

Figure 3.18 - Drainage segments of a drainage network are shown in different colors

Figure 3.19 gives an example of the subwatersheds delineated from an initial point in the drainage network (cyan), where each drainage segment determines a subwatershed, i.e., the area around the drainage segment which includes all the points that contribute to the flow accumulated in the nodes of the drainage segment. It is worthy mentioning that the number of delineated subwatersheds depends on the number of existent drainage segments, which in turn depends on the drainage network generated from a particular threshold value of accumulated flows. 


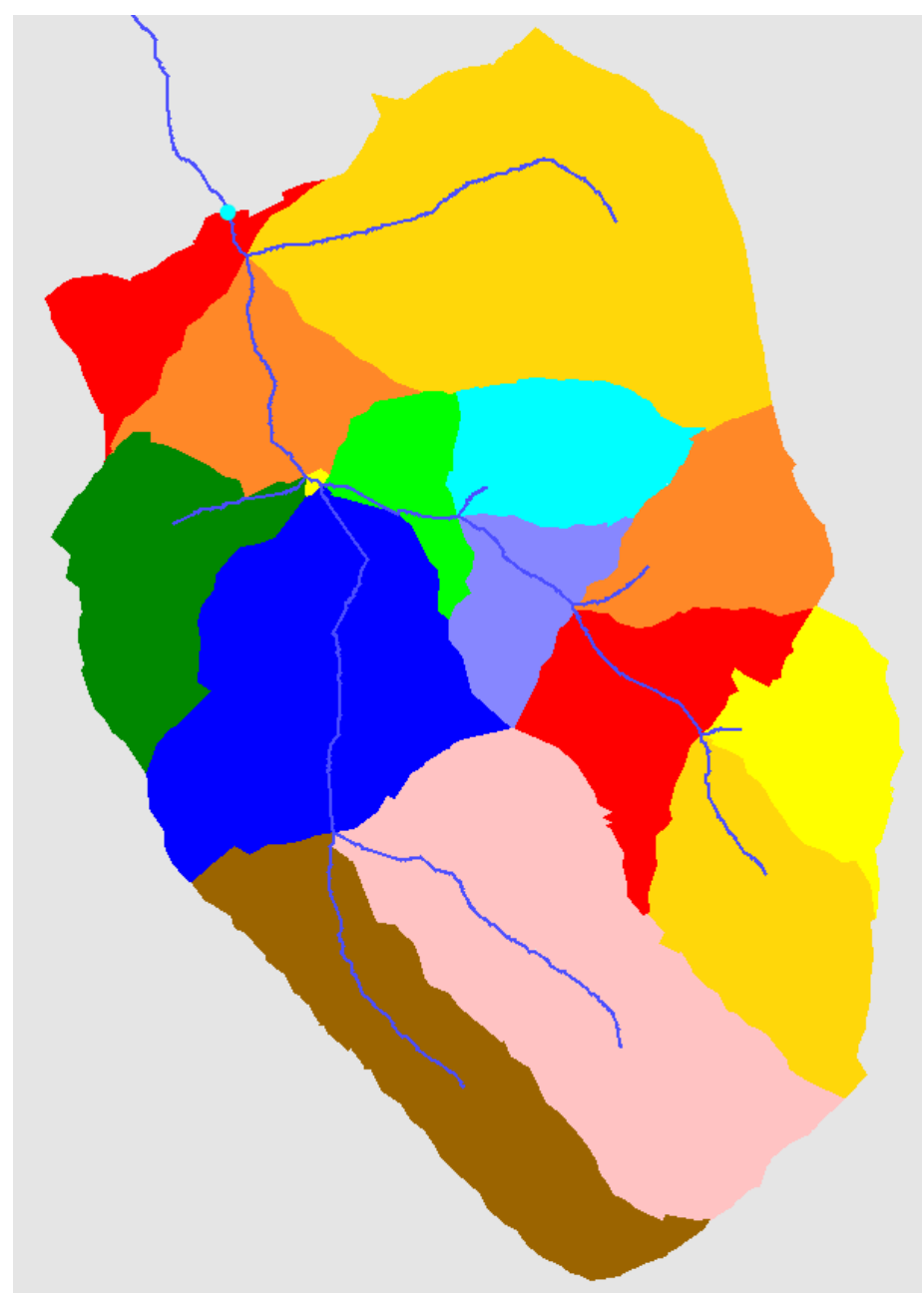

Figure 3.19 - Subwatersheds delineated from each drainage segment 


\section{RESULTS}

\subsection{Area of study}

The input data used in this work comprises contour lines and sampled points from an area located in the city of São José dos Campos - Brazil. All the data is irregularly distributed over the geographic region with bounds ranging from 396000.0m to $427400.0 \mathrm{~m}$ West and from $7421000.0 \mathrm{~m}$ to $7445000.0 \mathrm{~m}$ South, which are given in UTM coordinates and SAD69 projection. These UTM coordinates correspond to the geographic coordinates ranging from $-46.017^{\circ}$ to $-45.709^{\circ}$ in longitude and from $-23.317^{\circ}$ to $-23.102^{\circ}$ in latitude. Figure 4.1 exhibits a RapidEye image from 2011 (BLACKBRIDGE, 2008) of the geographic region that defines the area of study selected for the present work, given in UTM coordinates.

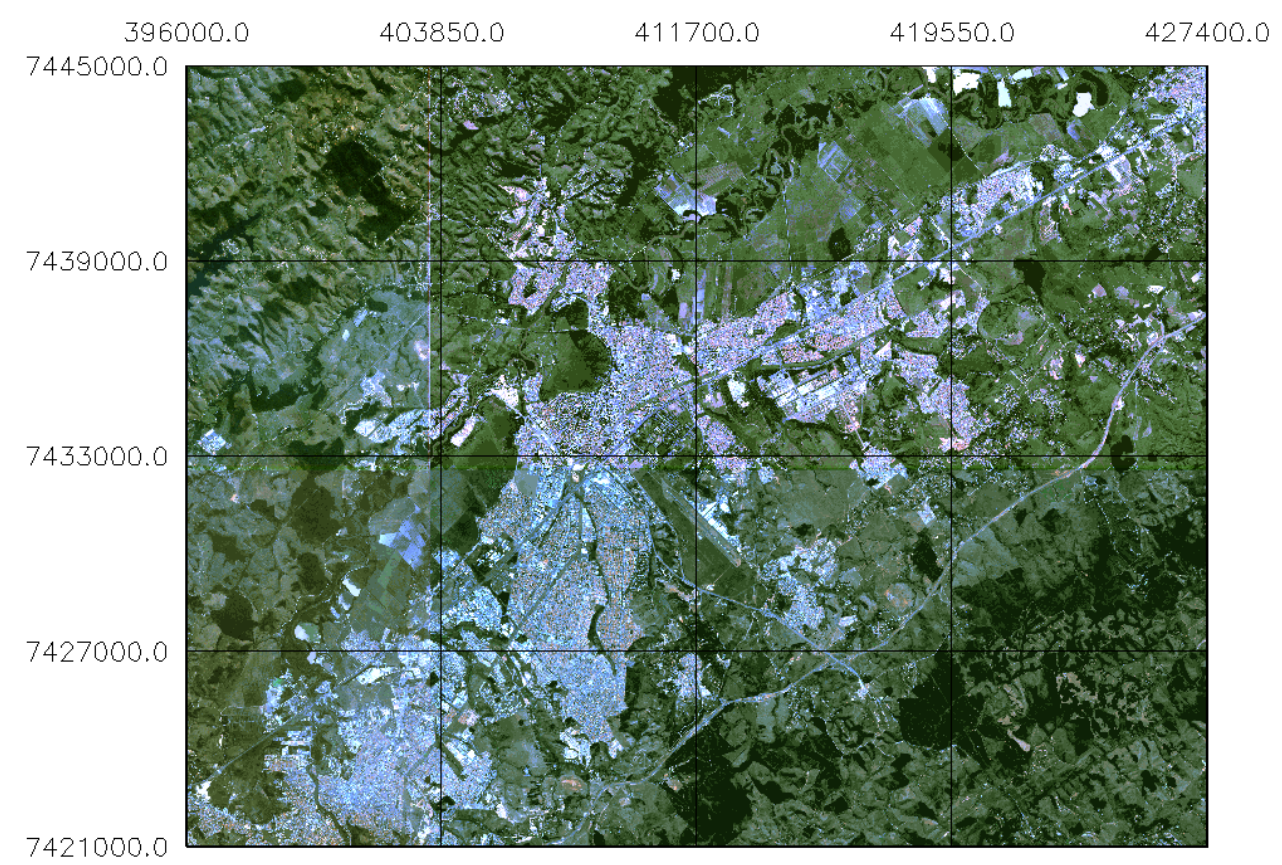

Figure 4.1 - Image from the area of study

Although the entire area of study is represented by the region described before, all the input data, i.e., the contour lines and sampled points, presents a higher density of information inside the region bounded by the coordinates ranging from $416940.0 \mathrm{~m}$ to $425290.0 \mathrm{~m}$ West and from $7428200.0 \mathrm{~m}$ to $7442400.0 \mathrm{~m}$, in UTM coordinates. Therefore the most detailed hydrological results generated in this work were considered mainly for this region since the TIN contains more triangles and, consequently, a richer set of drainage paths is calculated. 


\subsection{Reference drainage network}

In the present work, the drainage networks generated from the TIN terrain model by using the methods described in the preceding chapter are qualitatively compared to a drainage network available in the 'Cidade Viva' database (PREFEITURA MUNICIPAL DE SAO JOSE DOS CAMPOS, 2003).

The 'Cidade Viva' drainage network was manually produced by specialists from an analysis of contour maps and it represents the main courses of water over the area of study, so that it is considered here as a reference. Figure 4.2 exhibits in blue the reference drainage network over a RapidEye image of the region previously mentioned which presents a higher density of terrain information.

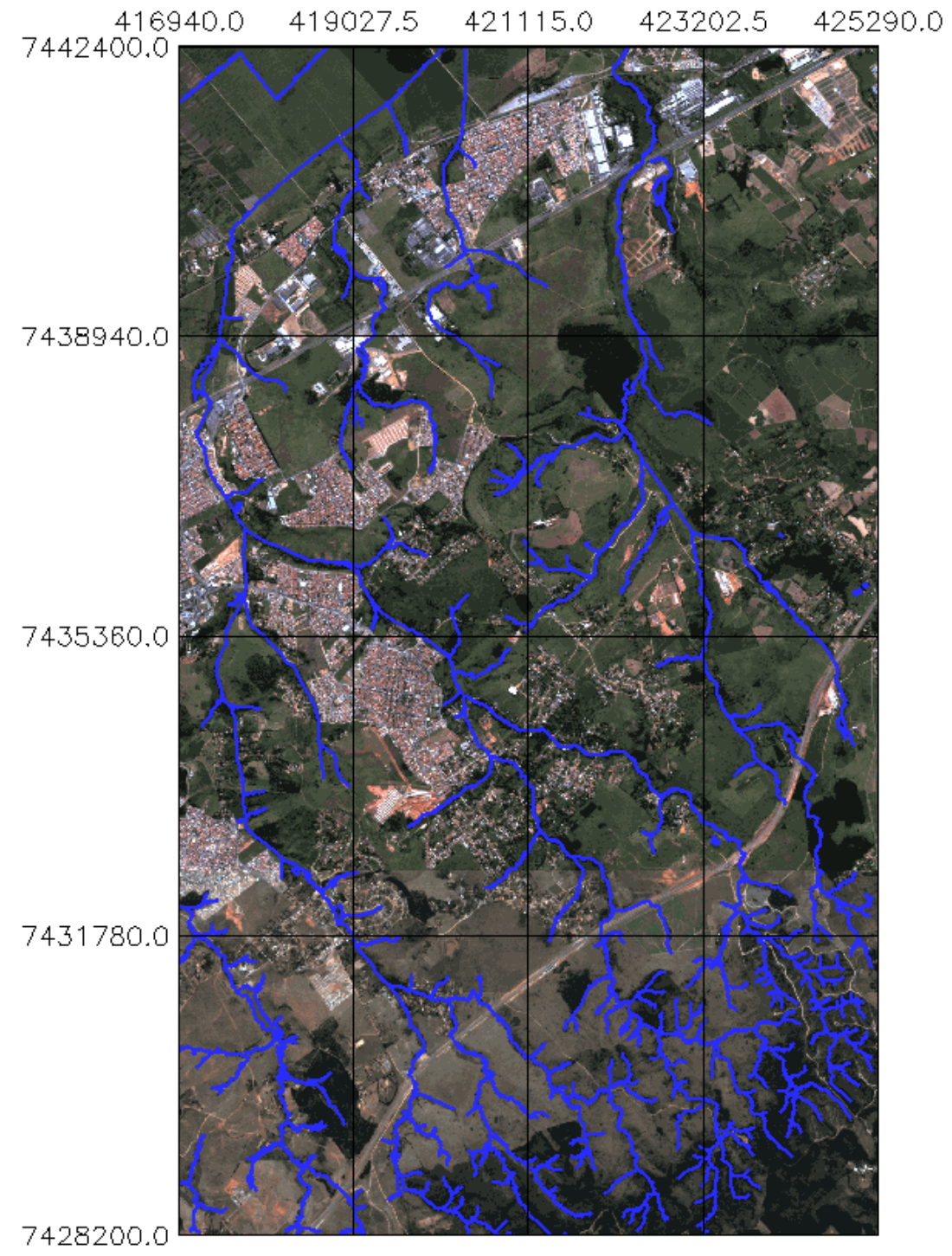

Figure 4.2 - Reference drainage network from the 'Cidade Viva' database 


\subsection{TIN results}

The main and most important results of this work generated from the TIN terrain model are represented by the hydrological structures defined from the gradient method (JONES et al., 1990) as described in the previous chapter. These structures constitute the drainage paths, drainage graph, drainage networks, watersheds and subwatersheds, where the drainage paths determine the basis structure for the generation of the others.

\subsubsection{Drainage paths}

Drainage paths can be visualized in figure 4.3 together with the reference drainage network for a small region from the area of study. It is possible to notice that the drainage paths approximately converge to the reference drainage network, thus forming drainage patterns very close to the real hydrological processes determined by the terrain surface.

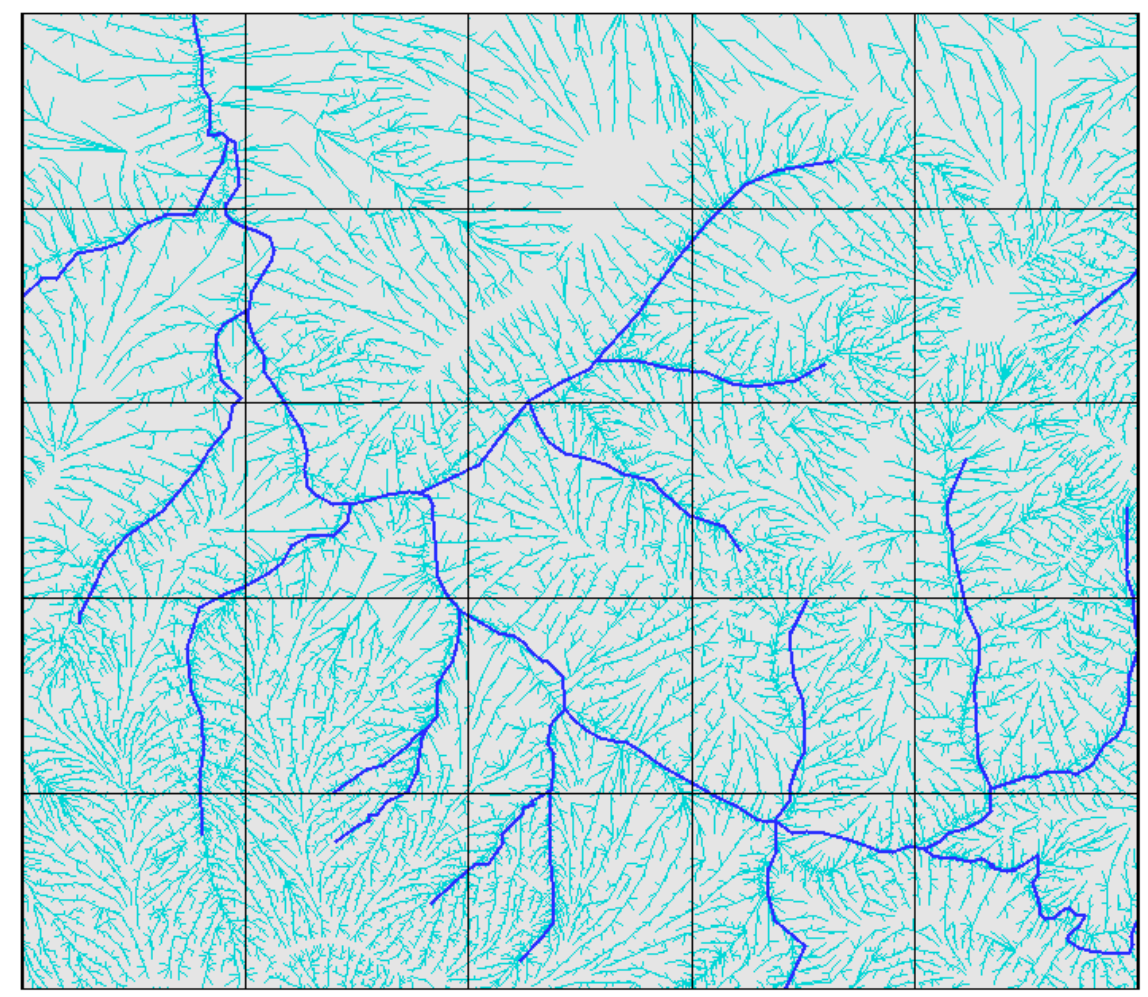

Figure 4.3 - Drainage paths (cyan) and reference drainage network (blue)

Discrepancies between the two drainage patterns, i.e., the drainage paths and the reference drainage network, may be due to either the precision of the input data 
since it changes the flow directions from triangle to triangle, or to the subjective analysis performed by the specialists who determined the main courses of water over the terrain surface from contour maps of the area.

For a more precise visualization of how the drainage paths are distributed over the triangles of the TIN, a closer view at both the drainage paths and the triangulation is given in figure 4.4, which presents these structures for an even smaller region from the area of study.

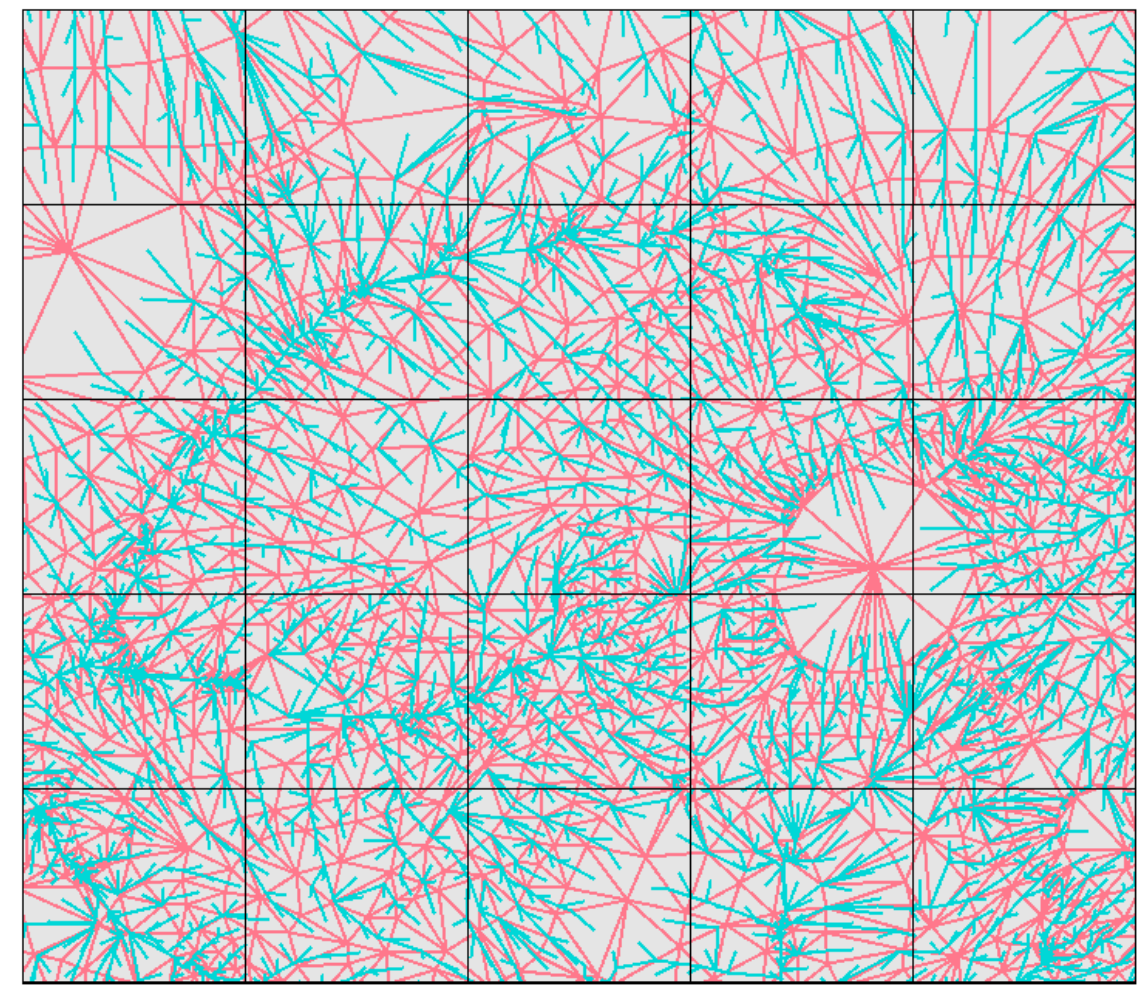

Figure 4.4 - Drainage paths over the TIN

\subsubsection{Drainage networks}

As mentioned earlier, this work presents procedures and algorithms that generate drainage networks from accumulated flows determined from the drainage graph structure detailed in the preceding chapter. Therefore the drainage networks are generated according to a threshold value compared to the value of the flow accumulated in each node of the drainage graph, so that flow segments are included as part of the drainage network only when their endpoints contain accumulated flows equal to or greater than the given threshold. 
With the availability of the drainage network from the 'Cidade Viva' database, which represents the main courses of water over the city of São José dos Campos, it is possible to perform a qualitative comparison between the reference drainage network and the drainage networks automatically generated from the drainage graph.

Figure 4.5 exhibits both drainage networks placed side by side for a visual comparison. It can be noticed that the main courses of water are really close, which indicates that high quality drainage networks, consistent with real-world drainage patterns, can be automatically generated from TIN terrain models. In this example, the TIN drainage network was generated from a threshold value of $40000 \mathrm{~m}^{2}$.

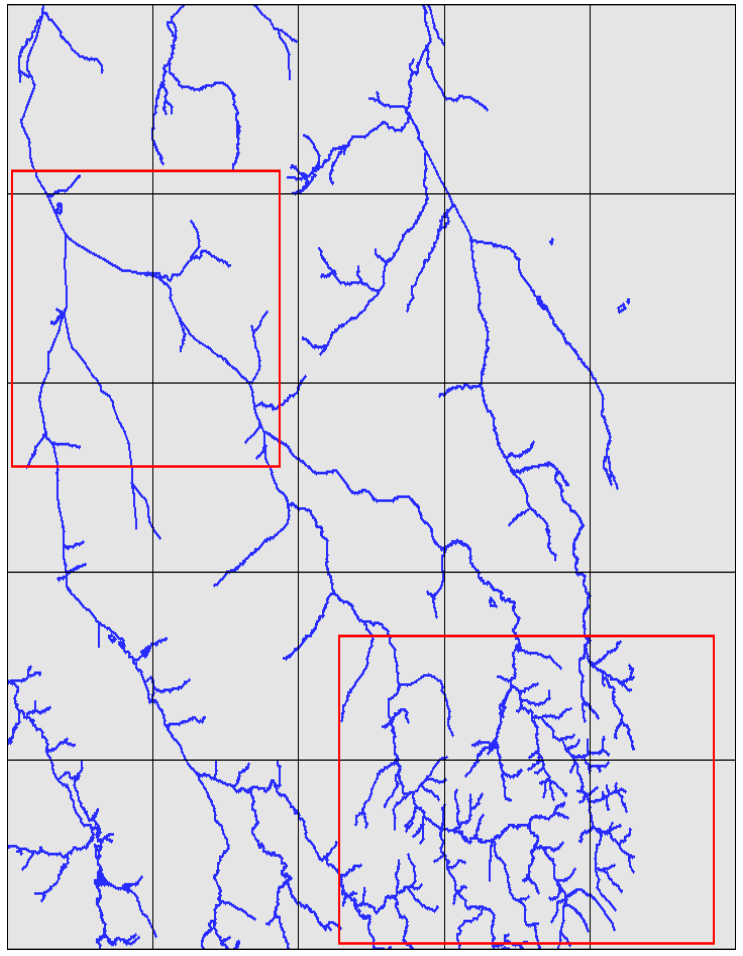

(a)

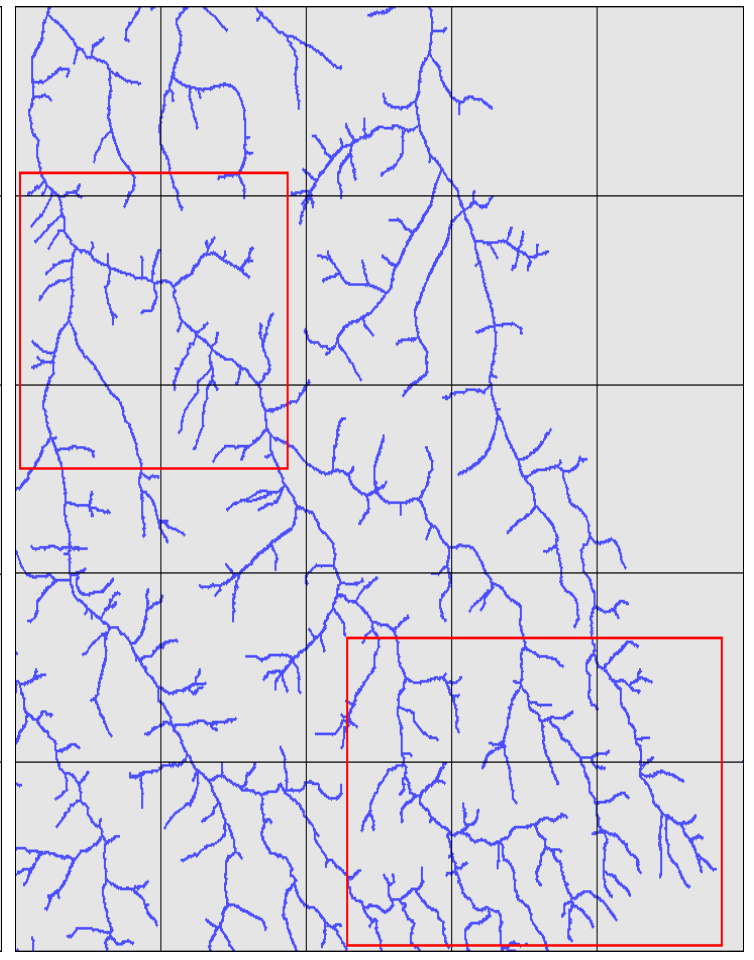

(b)

Figure 4.5 - Comparison between drainage networks: (a) reference drainage network (b) TIN drainage network

It is worthy mentioning that differences between the two drainage networks, when comparing the number of branches and drainage segments, occur due to the fixed threshold value chosen for the comparison with the accumulated flows used to generate the TIN drainage network, while the reference drainage network was produced from a subjective analysis of contour maps that represent the terrain model. Thus it is highly unlikely that both drainage networks can exactly match. 
The red rectangles in figure 4.5 highlight these differences, where the region indicated below presents a higher number of drainage segments in the reference drainage network while the TIN drainage network is not so dense, whereas the opposite can be seen in the region highlighted above.

\subsubsection{Watersheds and subwatersheds}

The watersheds and subwatersheds delineated from the TIN terrain model are represented by distinct polygons made up of the triangles that constitute the TIN. The triangles are considered as part of a watershed or subwatershed depending on the drainage paths that cross the triangles and also on the drainage segments present in the drainage network.

The procedure used to delineate the watersheds and subwatersheds from the drainage network by following the drainage paths was already described in the previous chapter. Figure 4.6 exhibits the watersheds and subwatersheds from three different regions in the southeast corner of figure 4.5(b), which are delineated from the initial points given in cyan located in the drainage network.

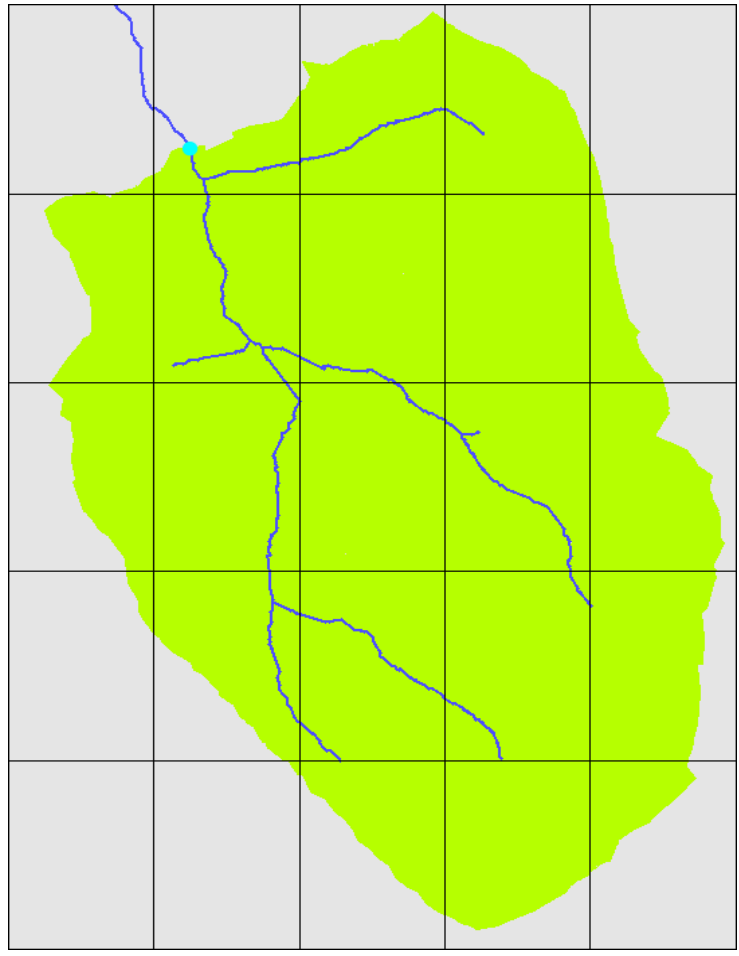

(a)

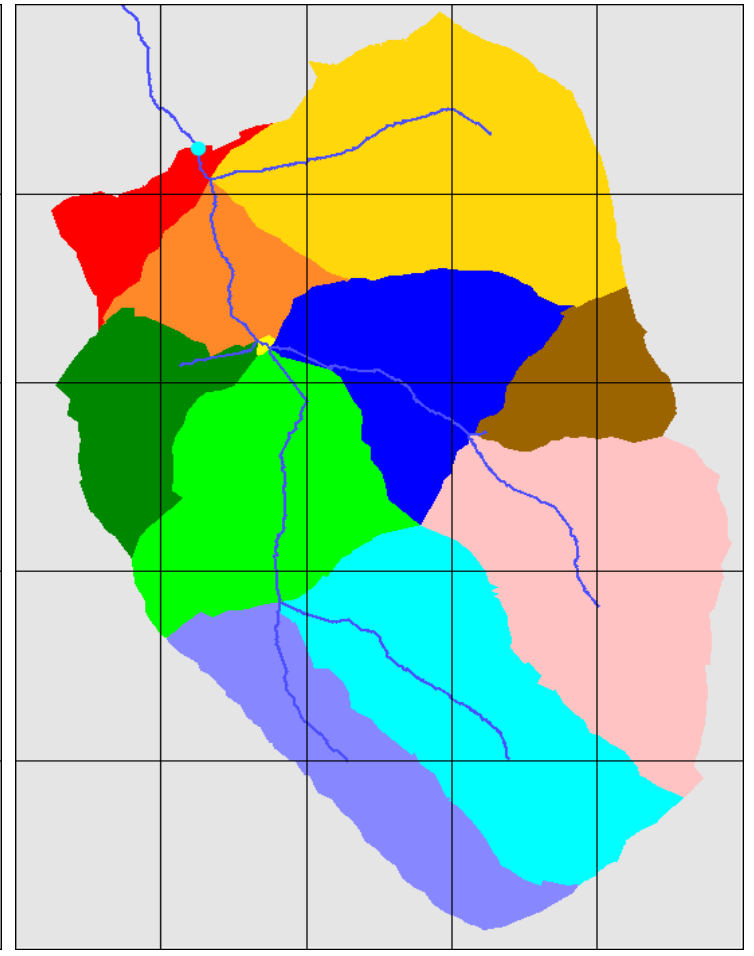

(b)

Figure 4.6 - Watersheds (left) and subwatersheds (right) delineated from the TIN (continue) 


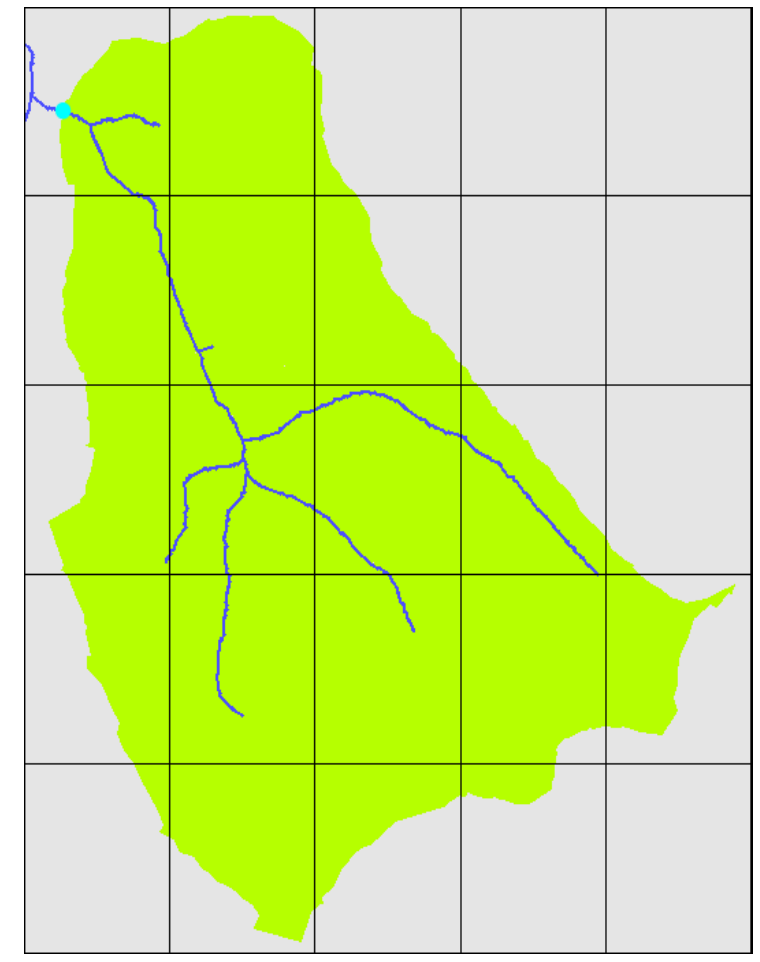

(c)

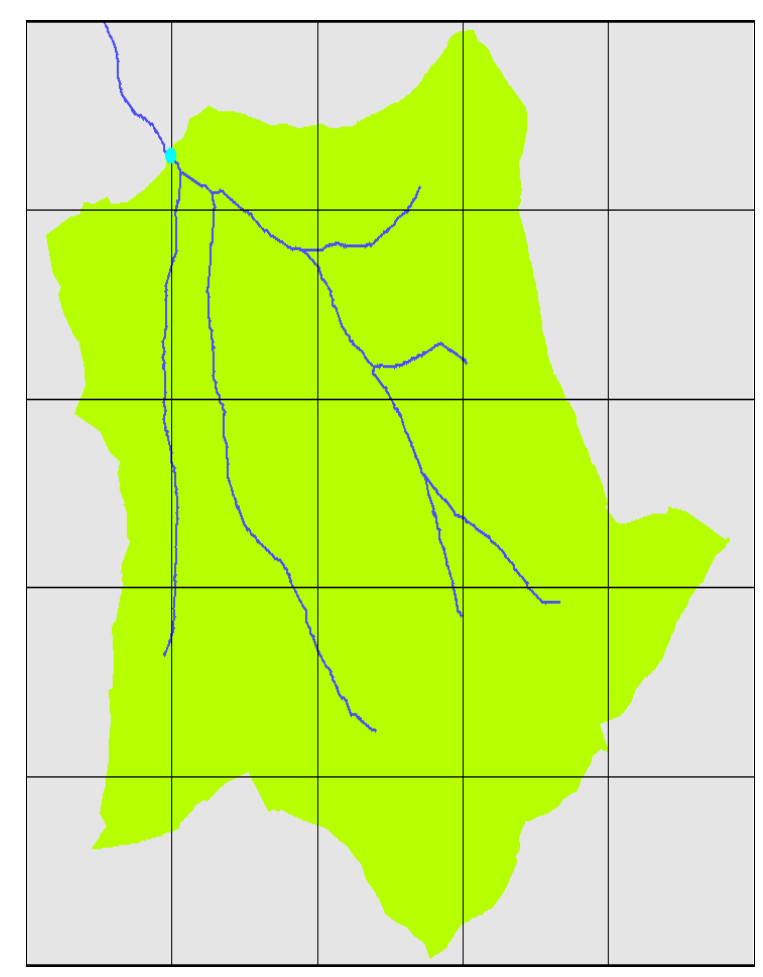

(e)

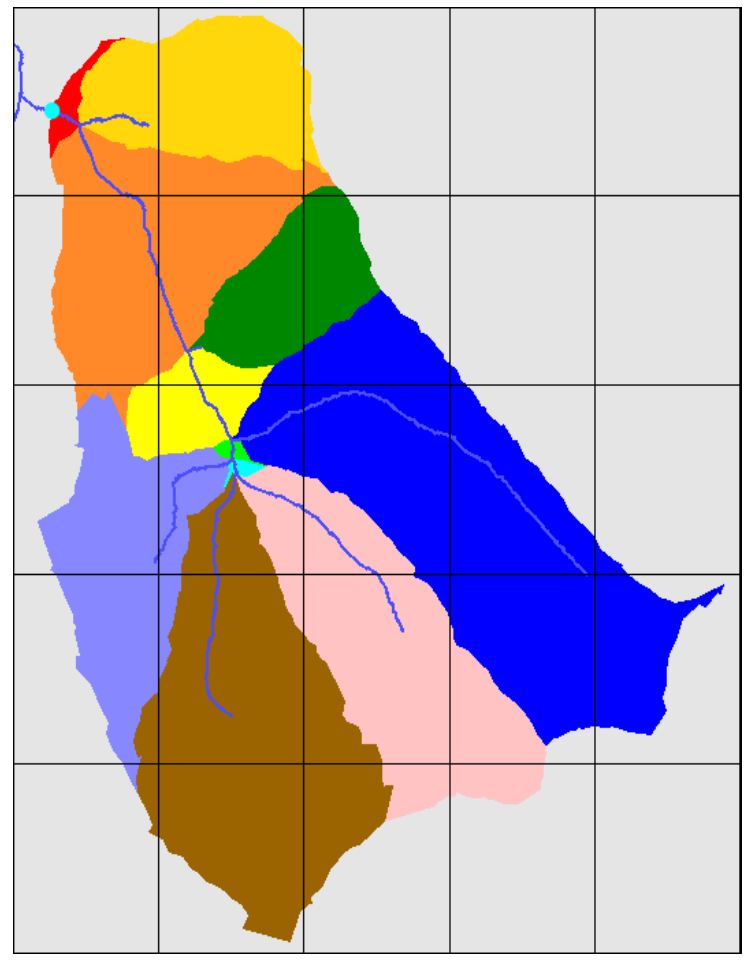

(d)

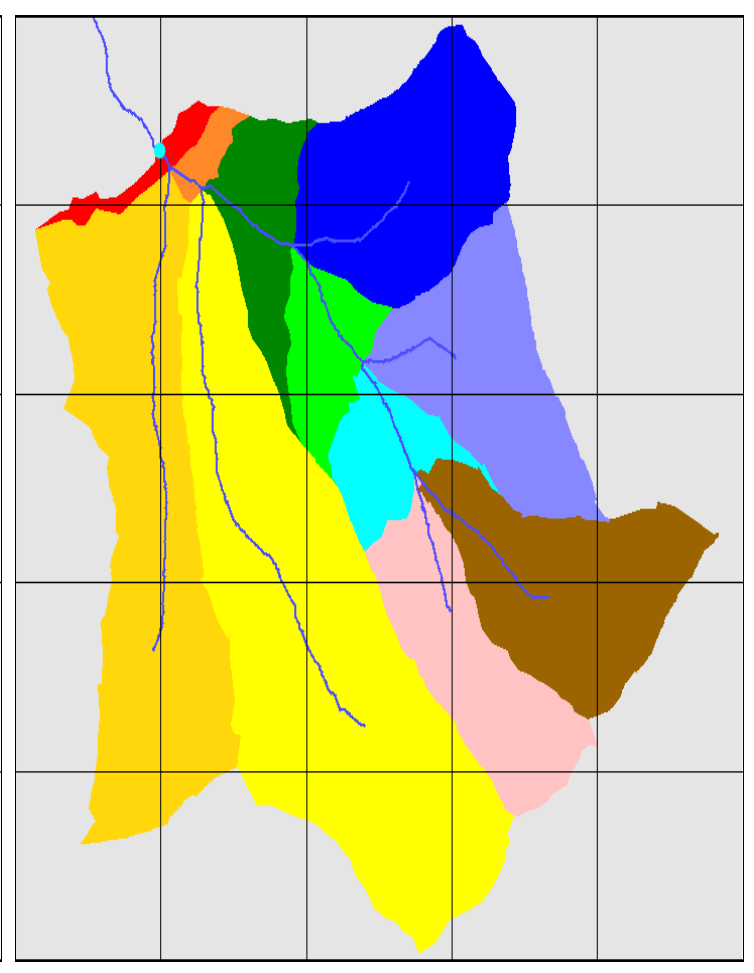

(f)

Figure 4.6 - Conclusion 


\subsubsection{Computational times}

Computational times are an important aspect related to the complexity of the procedures developed to generate hydrological structures from the TIN model. The algorithms presented in this work, in essence, perform linear-time procedures such as depth-first search (DFS) and breadth-first search (BFS) in order to solve common problems that may occur in terrain models, specifically, flat areas and pits.

These specific problems hinder the generation of the hydrological structures that, in this work, include the drainage paths used to define drainage networks from accumulated flows. As mentioned earlier, the drainage paths are recursively defined with DFS procedures performed over the TIN from either the edges or the vertices, which then determine inflows and outflows between points on the terrain surface.

As a practical example of the search algorithms, the problem of flat areas is solved by defining paths of triangles with a DFS procedure for a subsequent interpolation of particular points in the paths. This search procedure is performed for each corner triangle and branching paths are also processed afterwards. In addition, whenever a path of triangles is defined, information of the elevation values from different contour lines are readily obtained from neighboring triangles in order to avoid unnecessary search procedures, thus improving the computational time of the algorithm. This method for removing flat areas is fully explained in a previous chapter.

The computational times obtained from the algorithms described in this work are given in table 4.1 for different number of input points, together with the number of intersections between contour lines and triangles edges, the total number of triangles, flat triangles and pits in the final TIN, and also the number of nodes in the drainage graph. Each execution time was calculated as the average value of 3 from 5 program executions, where the minimum and maximum values were discarded.

Table 4.1 - Output information

\begin{tabular}{ccccccc}
\hline \hline Points & $\begin{array}{c}\text { Intersec- } \\
\text { tions }\end{array}$ & Triangles & $\begin{array}{c}\text { Flat } \\
\text { Triangles }\end{array}$ & Pits & $\begin{array}{c}\text { Drainage } \\
\text { Graph } \\
\text { Nodes }\end{array}$ & $\begin{array}{c}\text { Execution } \\
\text { Time (s) }\end{array}$ \\
\hline 51145 & 2398 & 152554 & 24804 & 209 & 307447 & 2.34 \\
100928 & 7469 & 267600 & 32421 & 179 & 539189 & 3.87 \\
150605 & 10446 & 397810 & 47623 & 164 & 802175 & 5.77 \\
202137 & 15861 & 514578 & 54644 & 12 & 1037775 & 9.02 \\
\hline \hline
\end{tabular}


It is worthy mentioning that all the algorithms were implemented in $\mathrm{C}++$ and compiled in a 64-bit machine, then the program was executed on a PC with Intel Core i7 $2.93 \mathrm{GHz} \mathrm{CPU}$ and $8 \mathrm{~GB}$ of RAM memory. The values shown in table 4.1 include the execution times of all the procedures listed next with the exception of the calculation of the original Delaunay triangulation as one considers that the Delaunay triangulation from a set of points is already given as input.

- Procedures computationally evaluated:
a) Constrained Delaunay triangulation;
b) Removal of flat areas and pits;
c) Re-triangulation with critical points;
d) Calculation of the plane gradient of each triangle;
e) Generation of the drainage paths;
f) Definition of the drainage graph structure;
g) Calculation of accumulated flows.

Moreover, the execution times given as a function of the number of input points can be visualized in figure 4.7 as a dispersion plot (blue), together with a fitting curve represented by a quadratic regression function (red). The quadratic polynomial curve generated from the points is defined by the equation included in the figure, which fits well to the data with $R^{2}$ coefficient equals to $0.9985^{1}$.

\subsection{TIN and regular grid results comparisons}

The most significant concern to be considered when analyzing the effectiveness of the methods developed in this work is the quality of the results generated after applying all the procedures to the TIN terrain model, i.e., the constrained Delaunay triangulation, the removal of flat areas and pits, and the generation of the drainage graph from the drainage paths with the subsequent calculation of drainage networks and delineation of watersheds and subwatersheds.

Therefore all the results are mainly assessed according to the hydrological consistencies of the drainage networks, watersheds and subwatersheds in order to determine if these structures are useful in real-world applications. A preceding

\footnotetext{
${ }^{1}$ If the $R^{2}$ coefficient of a regression curve derived from sampled data equals to 1 ., then it means that the curve perfectly fits the data (KIRK, 2008).
} 


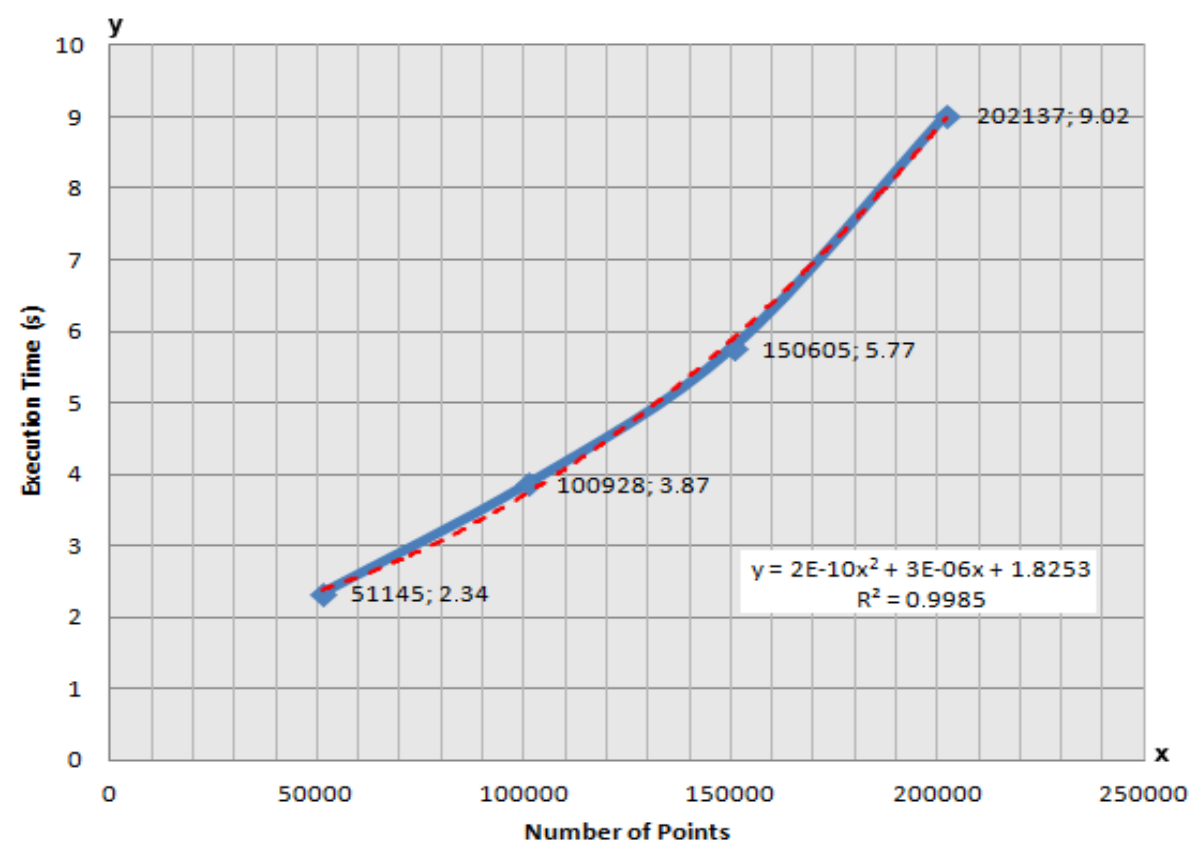

Figure 4.7 - Execution times for different number of input points

section already indicated that it is possible to generate drainage networks from accumulated flows over TIN terrain models with patterns very close to the courses of water from an available reference drainage network produced by specialists.

In addition, this work also considers as criteria of evaluation a qualitative comparison between the hydrological structures generated from the TIN with the methodology described here and from regular grids with the TerraHidro system (ABREU et al., 2012), which is a distributed hydrological modeling platform and part of the TerraView software (INSTITUTO NACIONAL DE PESQUISAS ESPACIAIS - INPE, 2010) developed at INPE.

It is important to mention that the regular grid used as input for the TerraHidro system was generated from the same set of contour lines and sampled points that comprise all the input data already presented in this work. The regular grid was generated with a method defined as weighted mean by quadrant and elevation, which is a regular grid interpolation functionality available in the Spring software (CAMARA et al., 1996) also developed at INPE. The grid cell resolution was defined as $5 \mathrm{~m}$, so the area of each grid cell is equal to $25 \mathrm{~m}^{2}$. 


\subsubsection{Drainage networks}

The drainage networks generated in this work are based on the accumulated flows calculated in either the nodes of the drainage graph, in the case of the TIN model, or the cells of the regular grid. In order to compare both drainage networks, the threshold values defined for the accumulated flows must be considered the same when processing the two structures, i.e., the TIN and the regular grid.

As the TerraHidro system generates drainage networks only from terrain models represented by regular grids, each flow direction then indicates how the water flows are accumulated from cell to cell. Thus a specific number of cells defined in a sequence of flows determines the total value of flow accumulated in each cell.

Figure 4.8 exhibits the drainage networks obtained from the TIN and from the regular grid for three different regions already shown in a previous section. The threshold value considered for the generation of the drainage networks in this example is equal to $40000 \mathrm{~m}^{2}$, which is equivalent to a sequence of 1600 grid cells, each one with an area of $25 \mathrm{~m}^{2}$.

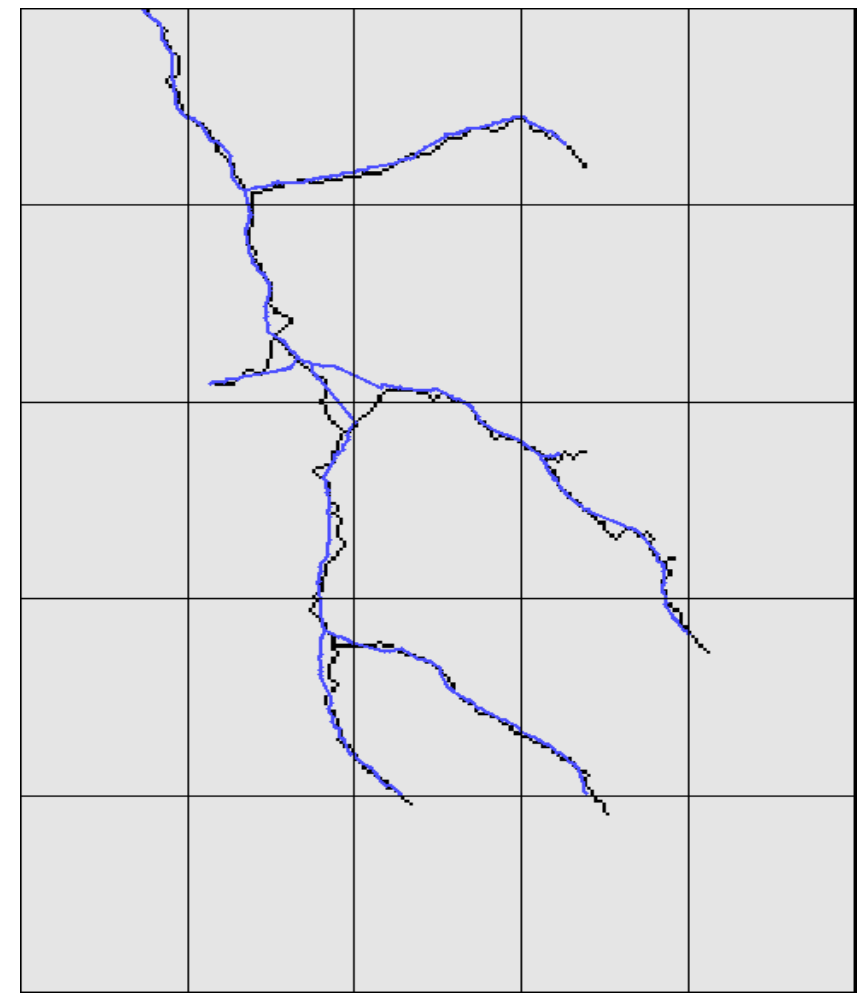

(a)

Figure 4.8 - Drainage networks generated from the TIN (blue) and from the regular grid (black) (continue) 


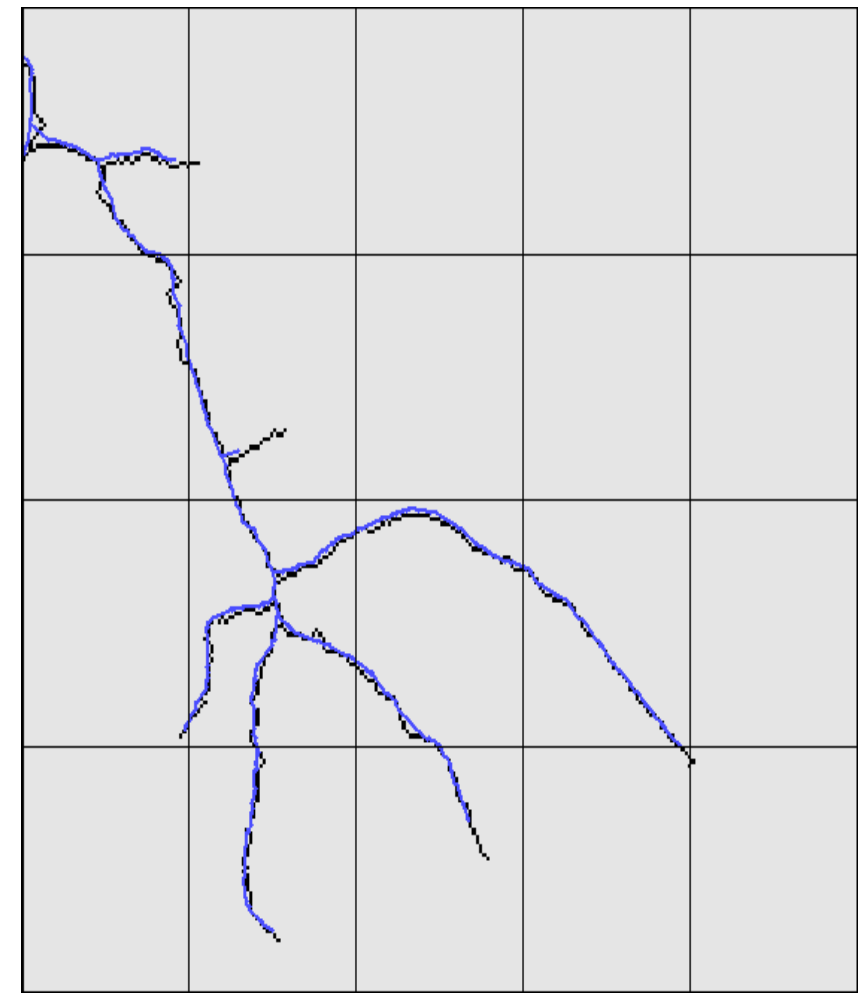

(b)

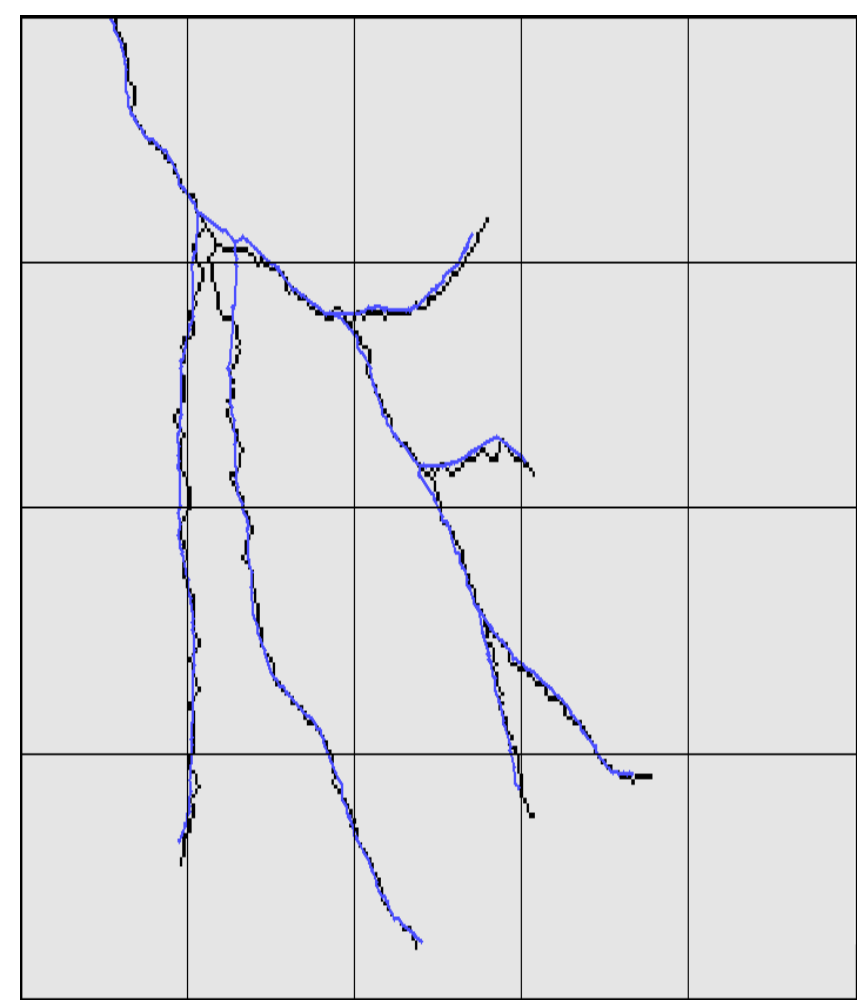

(c)

Figure 4.8 - Conclusion 
One can notice from figure 4.8 that the drainage networks generated from both terrain models present very close drainage patterns, which in turn represents a good indication that the hydrological processes derived from terrain surfaces defined by either a TIN or a regular grid reveal similarities between each other.

Another important consideration to be made, also regarding the results shown in figure 4.8, is that the drainage network generated from the TIN defines smoother paths relative to the paths from the regular grid. This occurs since the triangles from the TIN determine a better approximation of the terrain when compared to the regular grid, as already pointed out in a previous chapter. In a few words, as the flows in the TIN encompass all the possible directions while in the regular grid the flows are limited to only eight different directions, the drainage network defined over the TIN does not present rough variations along its extension.

Furthermore, from the results presented in figure 4.8, it can be noticed that there are some local discrepancies between the drainage networks from the TIN and from the regular grid due to the characteristics inherent to each structure. Specifically in regular grids, drainage networks can be affected by many factors such as, for instance, the interpolation method used for the generation of the regular grid when it is not readily available, the procedures performed to remove inconsistencies of flat areas and pits, and also how the flow directions are defined from cell to cell.

Figure 4.9 illustrates some examples of the differences between the two drainage networks. The yellow rounded shapes highlight parts where the drainage from the TIN follows smoother paths while the drainage from the regular grid takes unnecessary turns (figures 4.9(a), 4.9(b) and 4.9(d)), which possibly occur as a consequence of different flow directions defined over the TIN and the regular grid.

The red and green arrows in figure 4.9 indicate drainage segments that differ in size, which occurs due to either flow directions determining distinct drainage paths (figures $4.9(\mathrm{a})$ and $4.9(\mathrm{~d})$ ) or to different flow values accumulated at specific points of the drainage network (figures 4.9(b) and 4.9(c)), where low values produce longer segments while high values result in shorter ones.

Another type of difference consists in the case that, at very near locations, one drainage network presents branches while the other does not, as shown in figure 4.9 (b), where the drainage network from the regular grid contains branches, denoted by the blue arrows, while the one from the TIN follows only one direction. This occurs as a result from different accumulated flows between the TIN and the regular grid. 


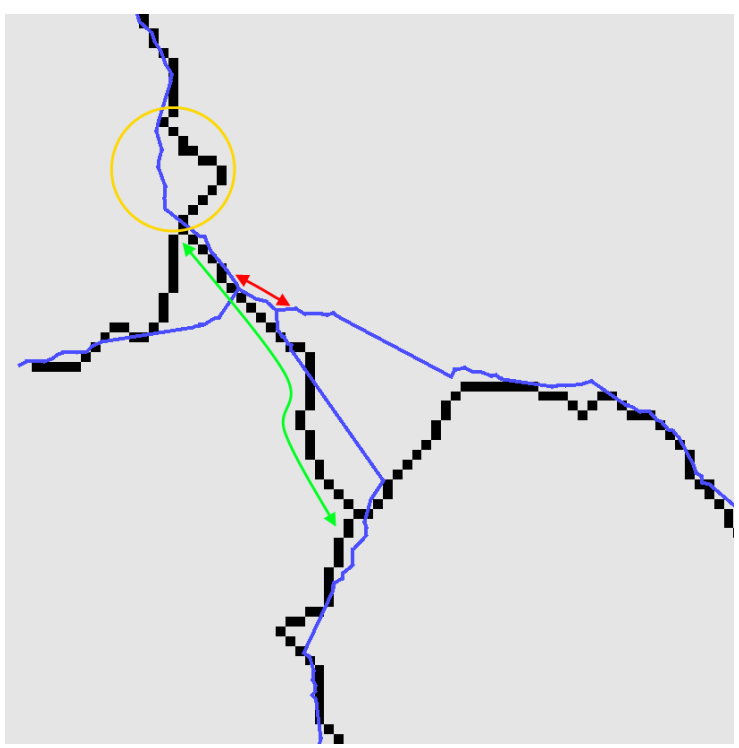

(a)

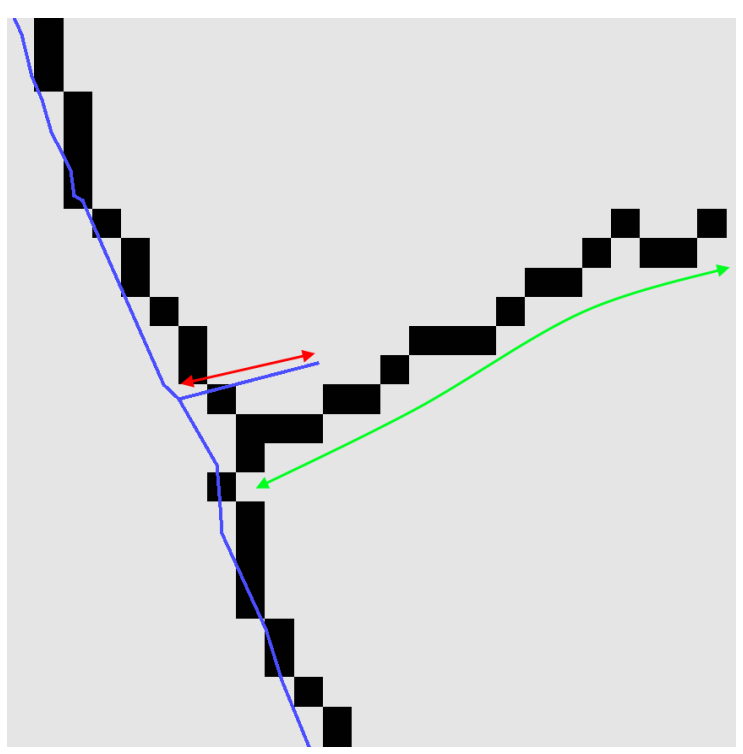

(c)

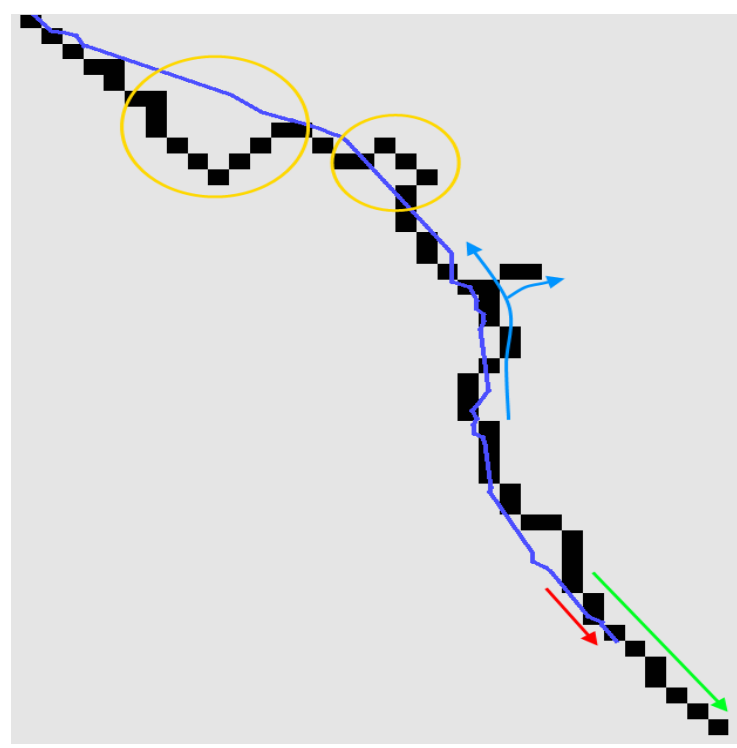

(b)

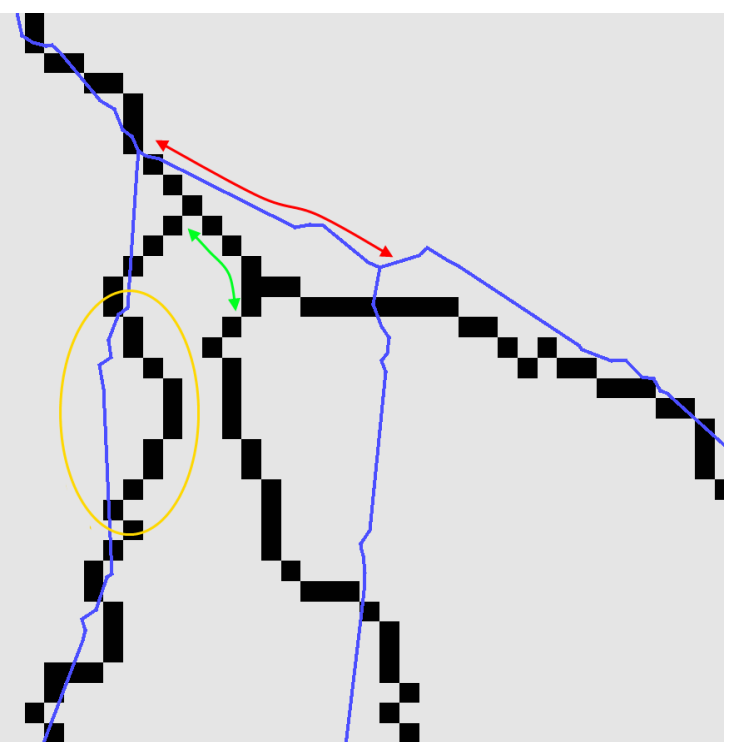

(d)

Figure 4.9 - Differences between the drainage networks from the TIN and from the regular grid 


\subsubsection{Watersheds and subwatersheds}

As already described in a previous section, the delineation of watersheds and subwatersheds of a given region depends on the drainage networks generated from the terrain model. Watersheds are delineated from initial points located in the drainage network considered as base points from where the flows are followed in the opposite direction, so that the upstream flow segments are recursively traversed to each reachable point until there are no more upstream flows to be followed. Similarly, the subwatersheds are delineated for each drainage segment of the drainage network, which are defined either between two branching points or one branching point and one ending point.

In this work, the watersheds and subwatersheds delineated from the TIN are qualitatively compared to the ones generated from the regular grid with the TerraHidro system previously mentioned. Figure 4.10 gives an example of the watersheds (figures 4.10(a) and 4.10(b)) and subwatersheds (figures 4.10(c) and $4.10(\mathrm{~d})$ ) delineated from the TIN and from the regular grid, by starting at the initial points given in cyan located in the two drainage networks.

The initial points particularly chosen from each terrain model do not present the same coordinates since the position of all the points that comprise the drainage networks, either from the TIN or from the regular grid, do not properly agree due to natural discrepancies that exist between both structures, as detailed in the preceding section. However, the initial points were selected to be as close as possible, i.e., the closest grid cell relative to the nodes of the drainage graph from the TIN.

It can be noticed from figures 4.10(a) and 4.10(b) that the delineated watersheds are very similar in size and shape, although some differences are clearly seen as indicated by the red circles, which occur due to distinct flow directions defined in the two terrain model structures, so that the areas around these small regions do not agree between each other as one structure may present more flows while the other presents less flows, or vice-versa.

Another significant difference between the two watersheds is that the borders from the TIN watershed present more continuous and smoother traces in relation to the borders of the regular grid watershed. This is a good indication that the TIN model generates more realistic representations of hydrological structures as already pointed out for drainage networks. 


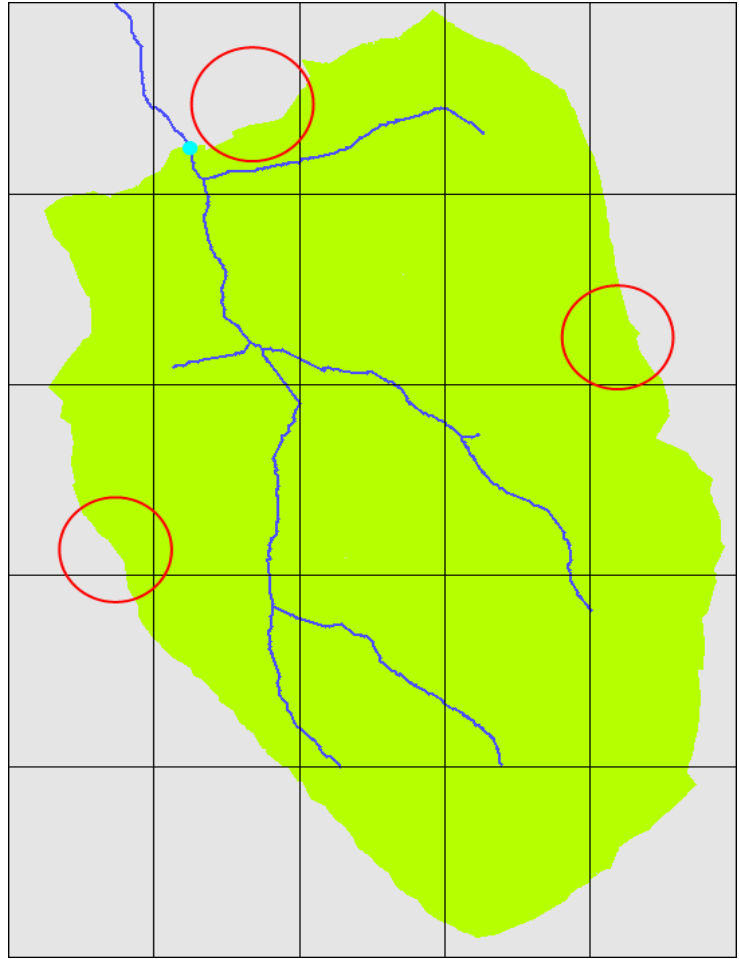

(a)

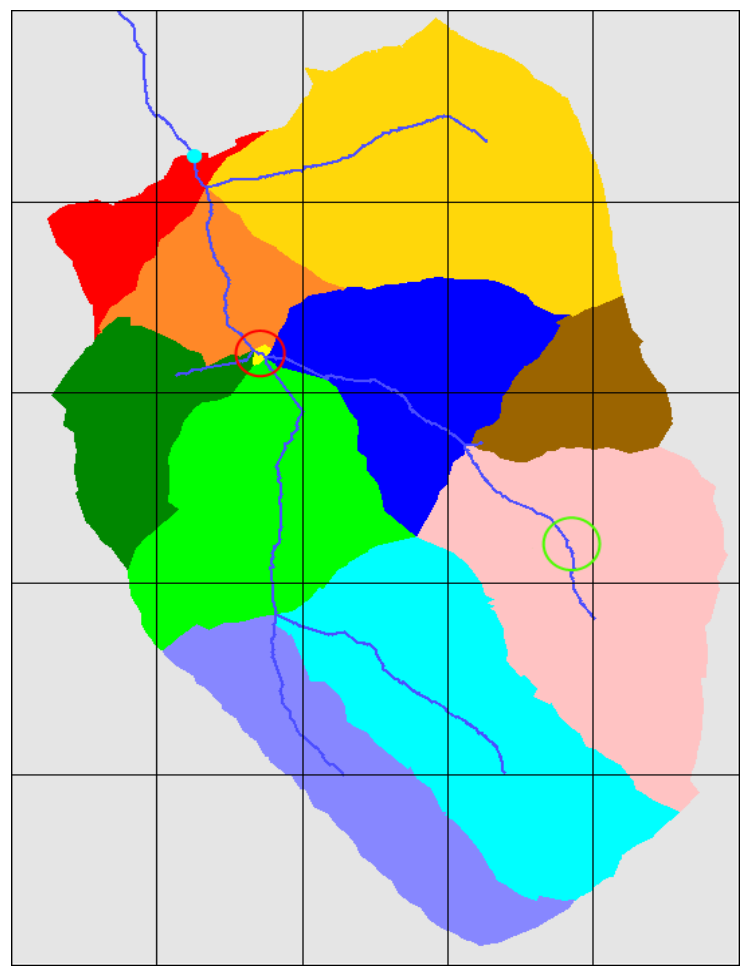

(c)

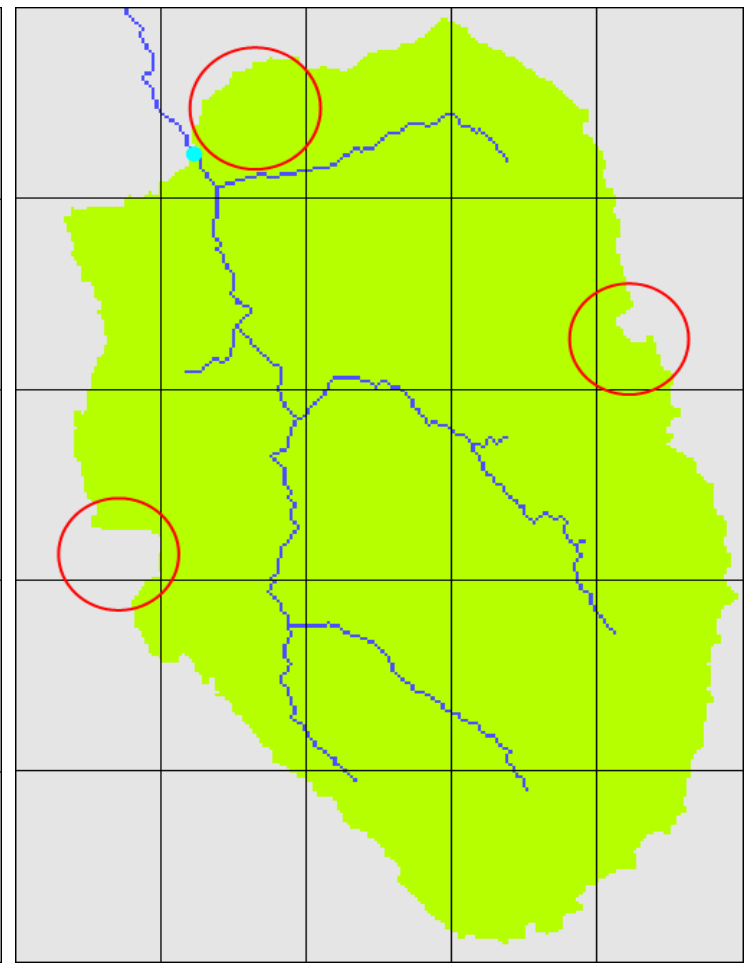

(b)

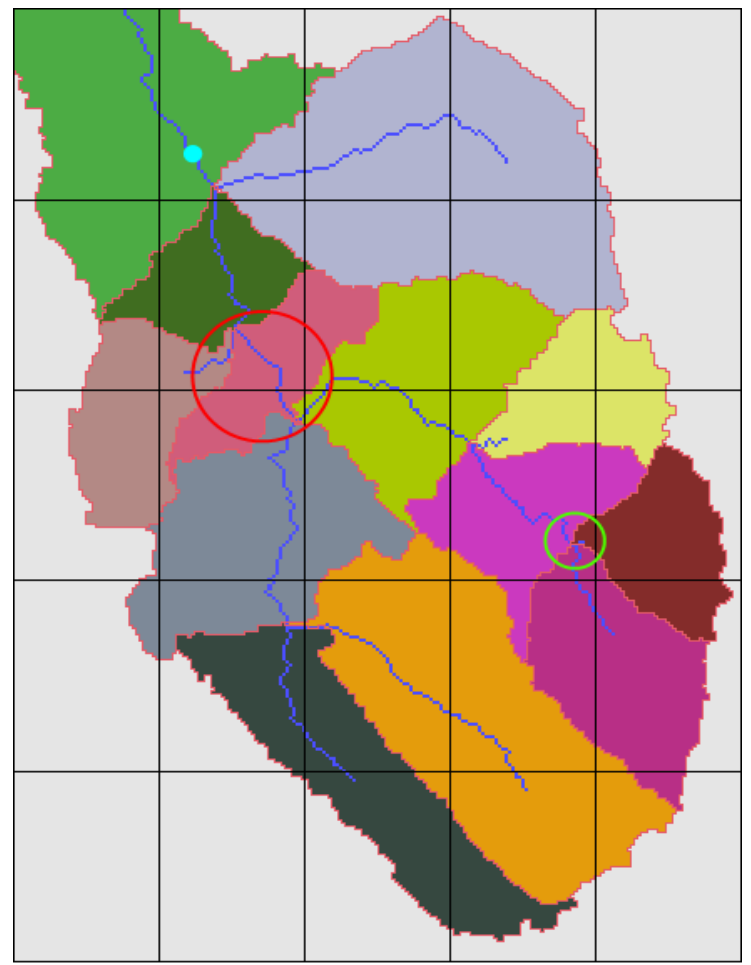

(d)

Figure 4.10 - Watersheds and subwatersheds delineated from the TIN (left) and from the regular grid (right) - Example 1 
Besides the aforementioned differences between the two watersheds, there are also differences between the subwatersheds generated from the corresponding TIN and regular grid drainage networks. Figures 4.10(c) and 4.10(d) indicate that most of the subwatersheds generated from each terrain model structure present similar sizes and shapes. However, differences between the two drainage networks, to be more specific, the distinct sizes of drainage segments as well as the presence of new drainage segments in only one of the drainage networks, result in the generation of totally disparate subwatersheds and new subwatersheds divisions, as indicated by the red and green circles. Figure 4.11 depicts these differences with more details.

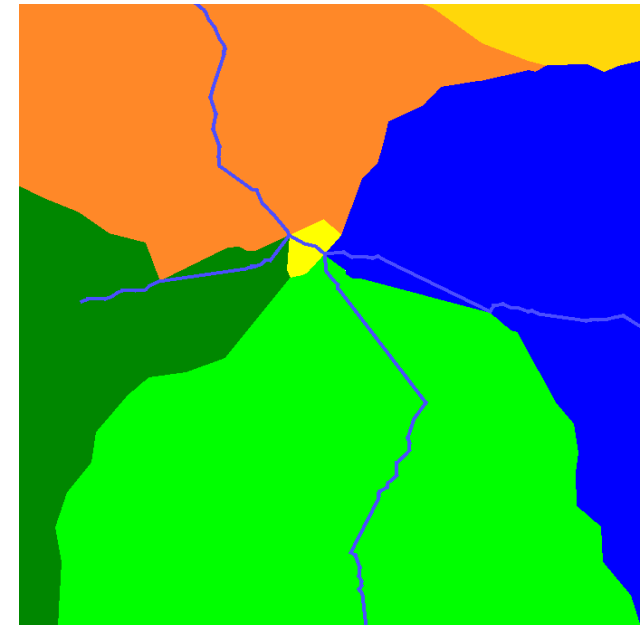

(a)

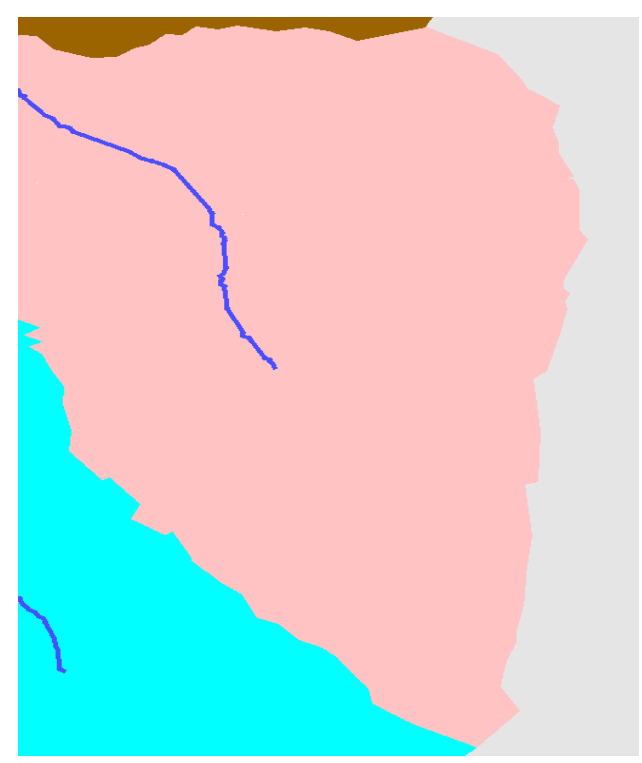

(c)

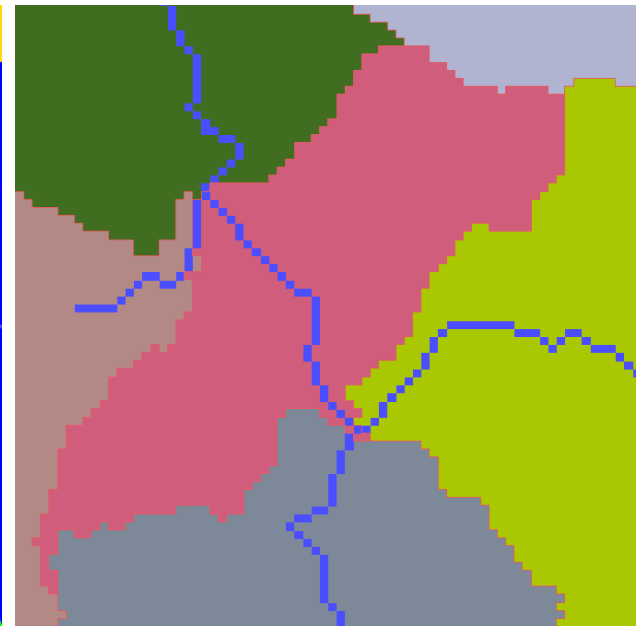

(b)

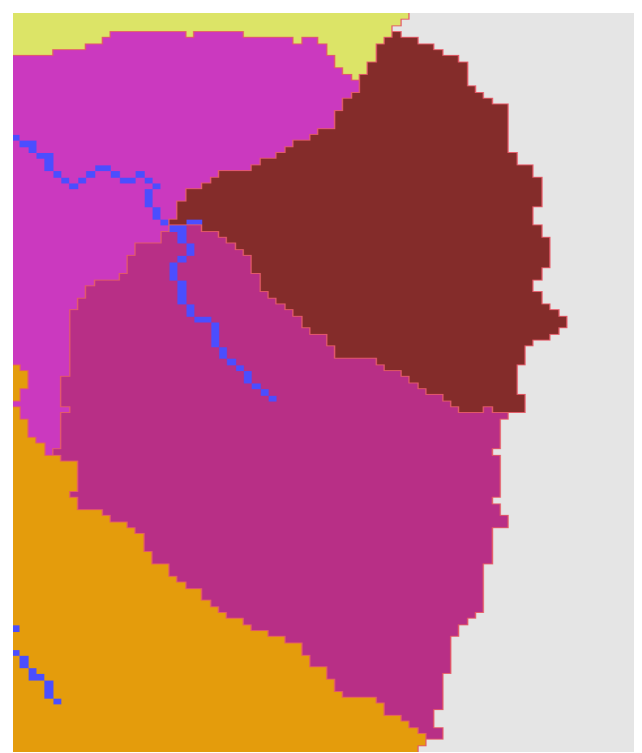

(d)

Figure 4.11 - Differences between the subwatersheds from the TIN (left) and from the regular grid (right) 
Additional illustrations of the differences between the watersheds and subwatersheds delineated from the TIN and regular grid drainage networks are shown in figures 4.12 and 4.13. These examples exhibit the hydrological results generated from distinct regions where the same types of problems pointed out in the last example also occur such as differences in the areas of some regions around the watersheds borders as well as subwatersheds with discrepancies in size and shape, both indicated by the red circles.

However, in these examples, the same number of subwatersheds was generated from each drainage network since all the drainage segments agree well with each other, so the corresponding pairs of subwatersheds are considerably very close patterns. The results shown in these figures reinforce that the TIN model can be used to derive really good approximations of the hydrological structures inherent to a terrain surface.

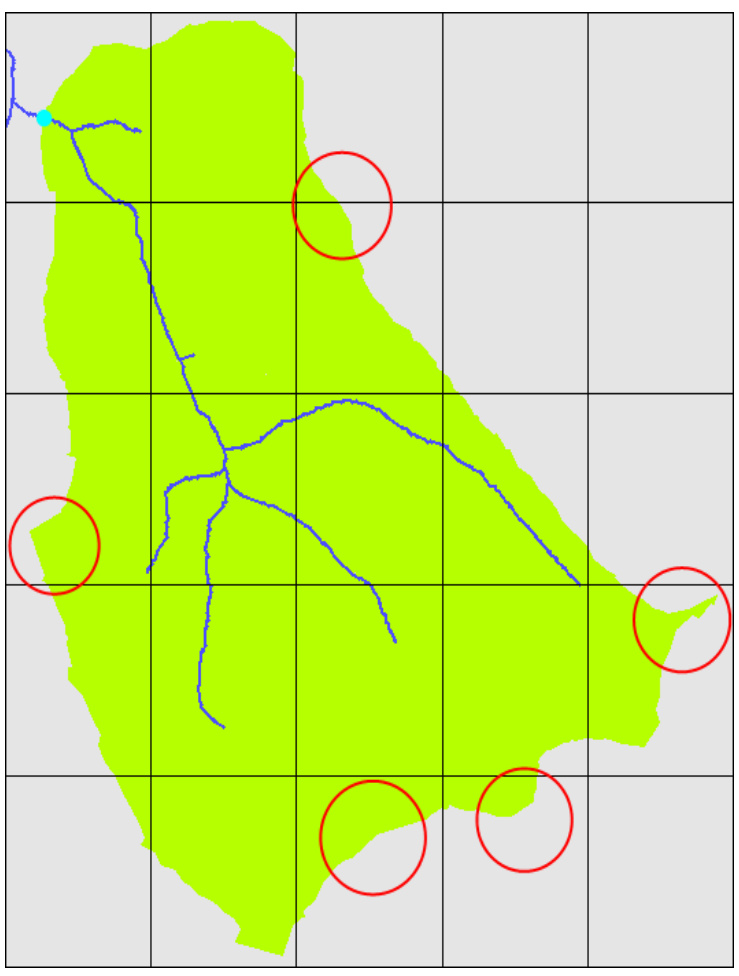

(a)

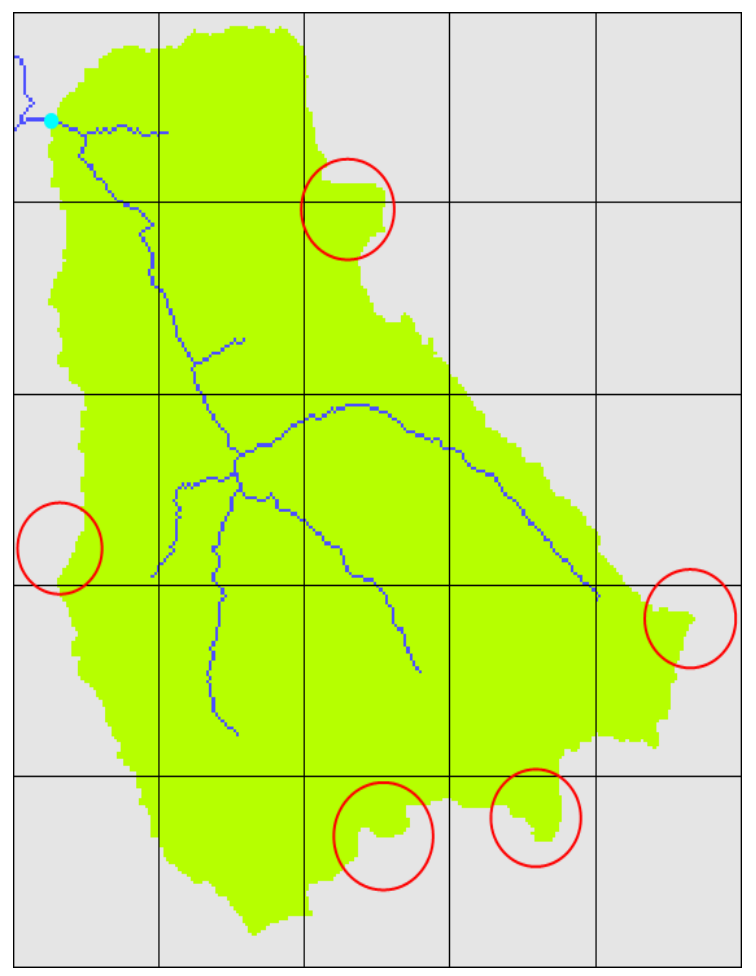

(b)

Figure 4.12 - Watersheds and subwatersheds delineated from the TIN (left) and from the regular grid (right) - Example 2 (continue) 


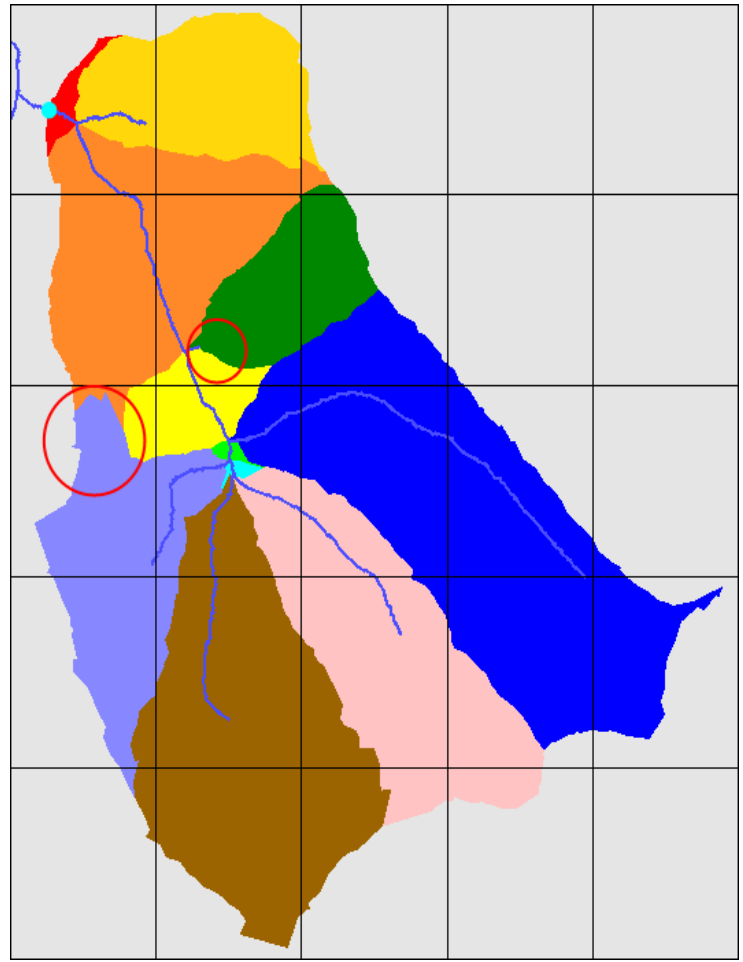

(c)

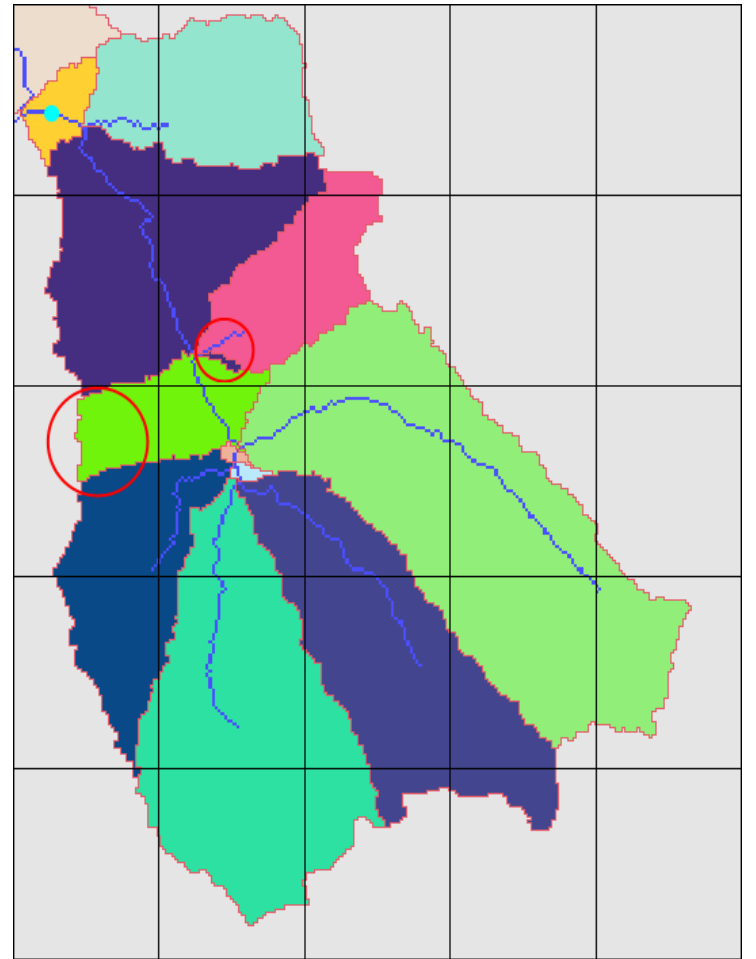

(d)

Figure 4.12 - Conclusion

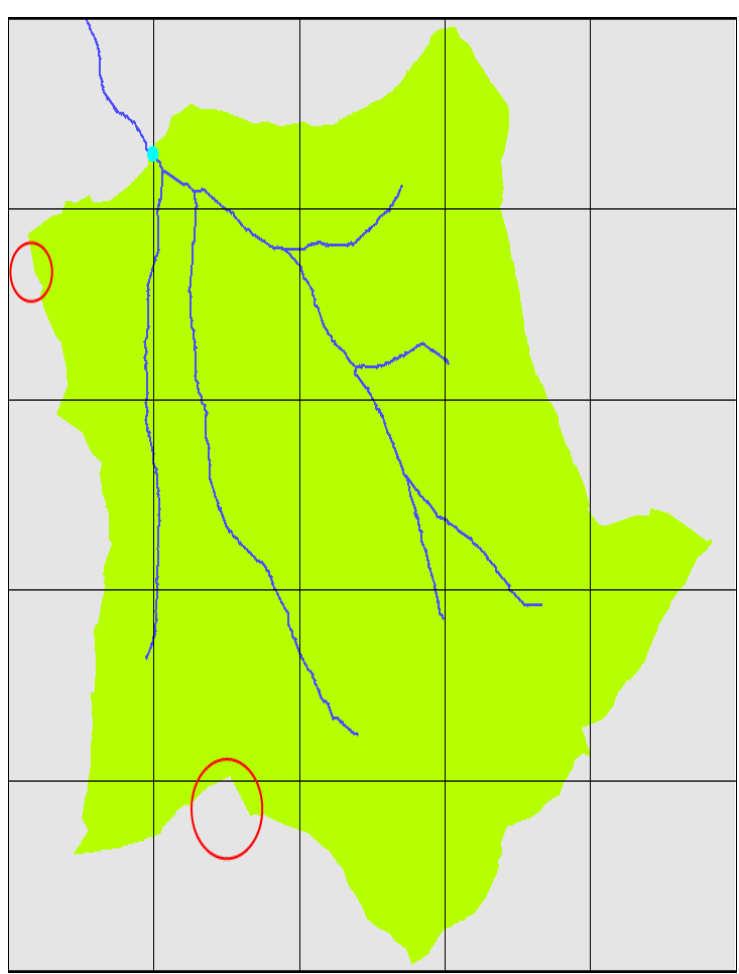

(a)

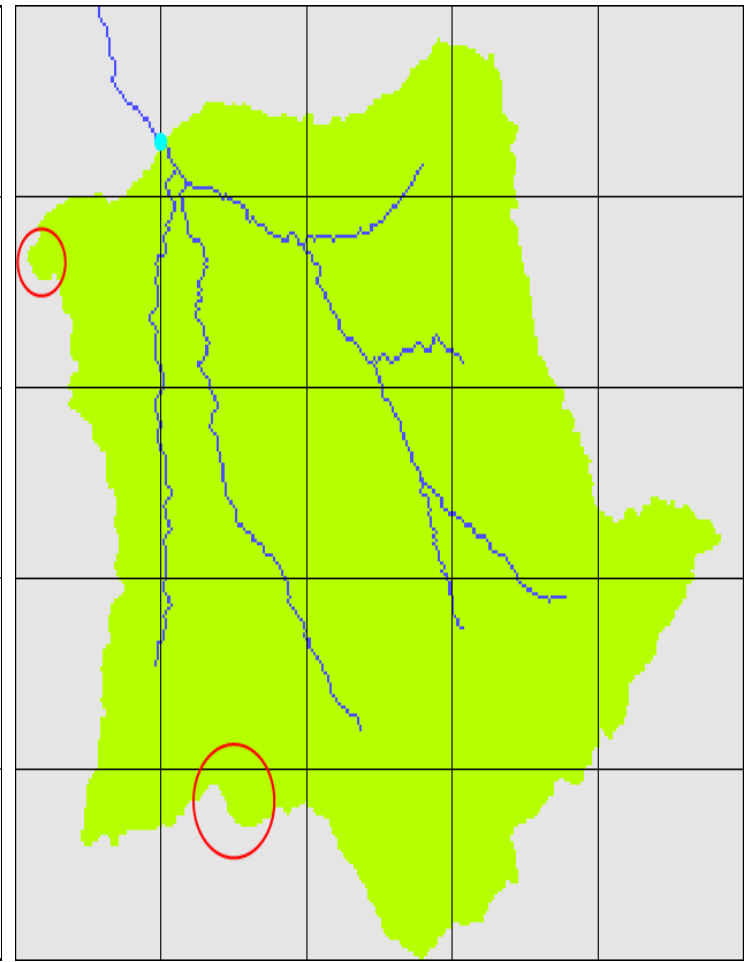

(b)

Figure 4.13 - Watersheds and subwatersheds delineated from the TIN (left) and from the regular grid (right) - Example 3 (continue) 


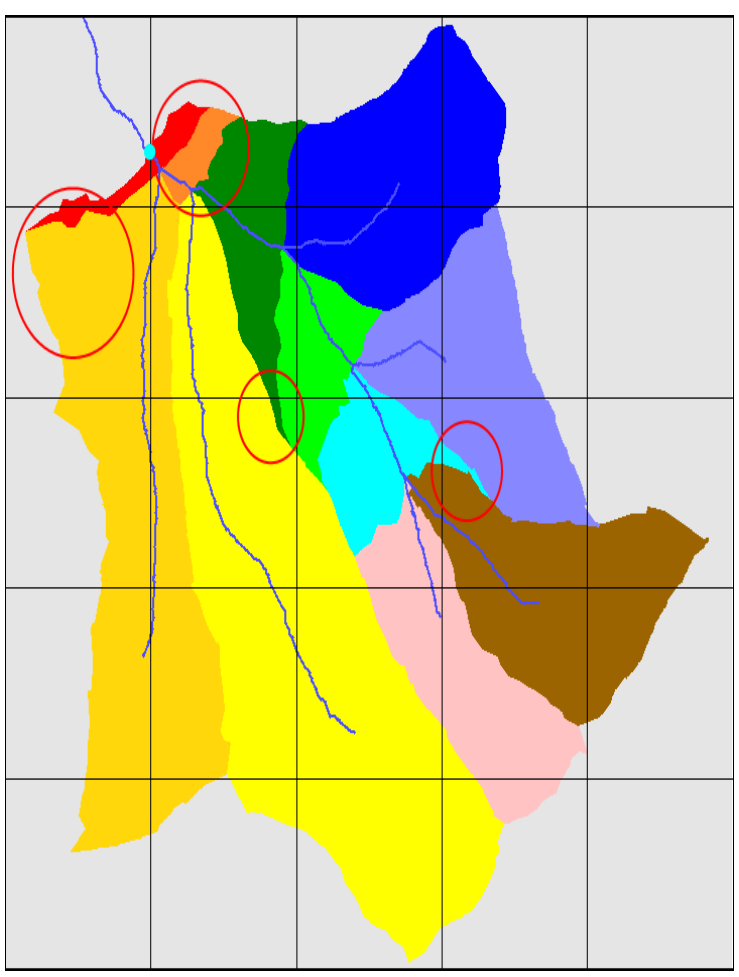

(c)

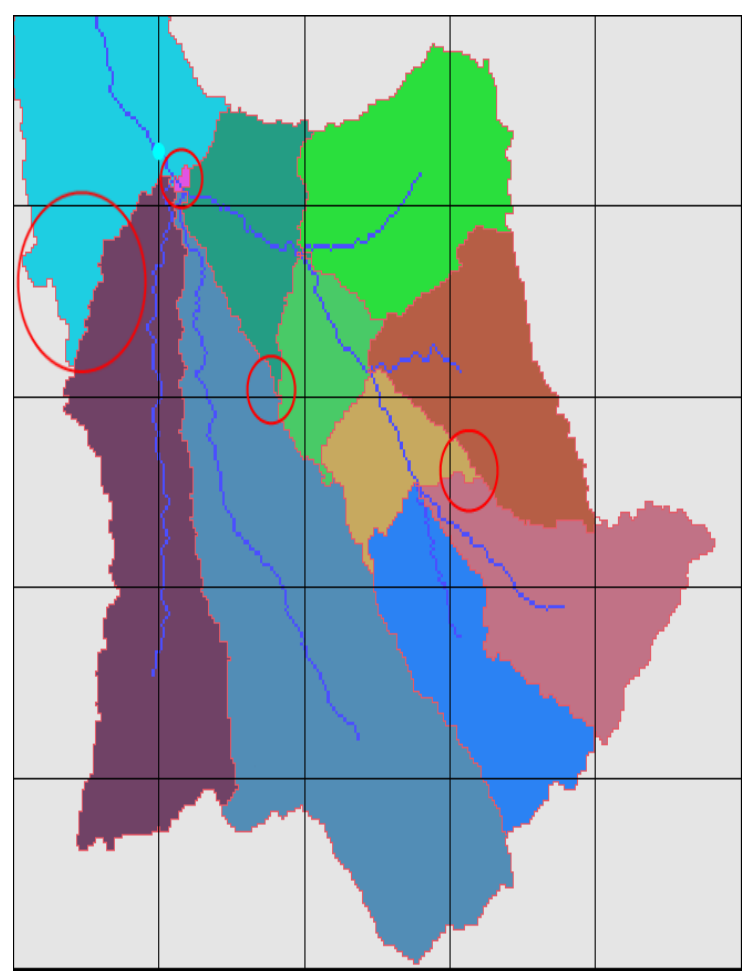

(d)

Figure 4.13 - Conclusion

\subsubsection{Computational times}

Still regarding the comparisons between TIN and regular grid results, the previous section included the computational times from the algorithms developed to process the TIN model. Additionally, the TIN computational times can be roughly compared to the computational times obtained from the regular grid processed in this work with the TerraHidro system.

Table 4.2 exhibits the same information already presented for the TIN together with the computational times obtained from regular grids of different resolutions and number of cells. The regular grid computational times include the execution times of all the procedures performed to remove terrain inconsistencies, namely flat areas and pits, as well as to generate the flow directions and accumulated flows.

One important consideration to be made about this comparison is that the TIN computational times depend mainly on the number of triangles since most of the procedures are performed over the TIN model, with the only exception of the accumulated flows which are calculated from the nodes of the drainage graph. Moreover, the regular grid computational times depend predominantly on the 
Table 4.2 - TIN and regular grid computational times

\begin{tabular}{|c|c|c|c|c|c|c|}
\hline \multicolumn{4}{|c|}{ TIN } & \multicolumn{3}{|c|}{ Regular Grid } \\
\hline Points & Triangles & $\begin{array}{c}\text { Drainage } \\
\text { Graph } \\
\text { Nodes }\end{array}$ & $\begin{array}{c}\text { Execution } \\
\text { Time (s) }\end{array}$ & $\begin{array}{c}\text { Cells } \\
\text { (Rows x } \\
\text { Columns) }\end{array}$ & $\begin{array}{c}\text { Cell } \\
\text { Resolution } \\
(\mathrm{m})\end{array}$ & $\begin{array}{c}\text { Execution } \\
\text { Time (s) }\end{array}$ \\
\hline 51145 & 152554 & 307447 & 2.34 & $\begin{array}{c}273452 \\
(548 \times 499)\end{array}$ & 20 & $\begin{array}{c}75.8 \\
(1 \min 15 \mathrm{~s})\end{array}$ \\
\hline 100928 & 267600 & 539189 & 3.87 & $\begin{array}{c}485450 \\
(730 \times 665)\end{array}$ & 15 & $\begin{array}{c}177.12 \\
(2 \min 57 \mathrm{~s})\end{array}$ \\
\hline 150605 & 397810 & 802175 & 5.77 & $\begin{array}{c}1091715 \\
(1095 \times 997)\end{array}$ & 10 & $\begin{array}{c}308.24 \\
(5 \min 8 \mathrm{~s})\end{array}$ \\
\hline 202137 & 514578 & 1037775 & 9.02 & $\begin{array}{l}4369050 \\
(2190 \times 1995)\end{array}$ & 5 & $\begin{array}{c}1848.86 \\
(30 \min 48)\end{array}$ \\
\hline
\end{tabular}

number of cells, although the lower this number the worse is the regular grid resolution.

From table 4.2 , it is possible to notice that a comparison between the execution times obtained from processing close number of triangles and grid cells such as, for instance, 267600 triangles and 273452 grid cells or even 514578 triangles and 485450 grid cells, indicate that the algorithms developed for the TIN model are likely to be more efficient computationally than for the regular grid.

\subsubsection{Quantitative comparison}

A major and rather complicated problem which follows from the analysis considered between the TIN and regular grid results is the difficulty to define specific measures that provide reasonable numerical results in order to determine how close or similar are all the hydrological structures derived from the TIN and from the regular grid.

A variety of criteria can be used such as the spatial distance between the closest points from each drainage network, the drainage area from accumulated flows, the length of the drainage segments, and the bifurcation and length ratios (MCCOY, 1970; AGRAWAL et al., 2006), which may constitute a more precise and complete comparison between the hydrological results generated from each terrain model, although these measures are not consolidated and not appropriate as numerical evaluations of the results, so that this work does not consider a quantitative comparison. 



\section{CONCLUSIONS}

This work considered the terrain model defined by a Triangulated Irregular Network (TIN) calculated from an input dataset comprised of contour lines and sampled points extracted from contour maps, which presented many different types of problems characterized as errors in the terrain model. These problems possibly occurred due to the whole set of input data be manually generated, even though fully devised by specialists. The errors present in the data hindered the generation of more exact results and had to be minimized with a careful visual and manual correction process in order to improve the quality of the terrain model, although the development of an automatic correction procedure is desirable.

Terrain inconsistencies such as flat areas and pits were removed with specific procedures that performed different search algorithms in order to get terrain information from surrounding contour lines and sampled points, where this information indicated how new elevation values are calculated and assigned to critical points inserted into the triangulation. As presented in this work, the specific use of neighboring terrain information by the procedures for removing flat areas and pits is considered an improvement over the existing methods that define paths of flat triangles and points, thus determining one important contribution.

The most promising and important result of this work is the generation of a drainage graph structure represented by a DAG that contains nodes determined from specific connections between the drainage paths defined over the TIN. The drainage graph allows the calculation of drainage networks from accumulated flows, as extensively used in regular grids, but still non-existent for TINs. Although some previous and recent works developed techniques to calculate drainage patterns over TINs, none of them defined specific structures and procedures for the calculation of accumulated flows and the generation of drainage networks that strictly follow the paths of steepest descent.

The use of the TIN model for hydrological purposes with the methodology developed in this work generated consistent and useful hydrological results. Drainage paths traced by starting at each triangle centroid, ordered by their elevation values and determined from the flow directions given by the gradient of the triangles, closely converged to the main streams of a reference drainage network produced by specialists. In addition, the watersheds and subwatersheds delineated from the drainage networks generated from the drainage paths reasonably represented the areas that drain water to specific locations on the terrain. 
The procedures proposed and developed for processing the TIN in order to remove terrain inconsistencies of flat areas and pits as well as to define the drainage paths from the gradient of each triangle, and also with the subsequent calculation of hydrological structures such as drainage networks, watersheds and subwatersheds, all presented low computational times since these procedures are mostly based on lineartime algorithms. This observation particularly indicates that the TIN structure can be considered as an attractive alternative to other terrain models, specially for hydrological applications.

It is important to mention that this work also presented a qualitative comparison between the hydrological structures generated from the TIN and from the regular grid. All the hydrological results from each terrain model structure were considered very close, although presenting discrepancies between one another that were expected to occur. Most of the comparisons were made only between the resulting drainage networks which were remarkably close with relation to the quantity and lengths of the drainage segments as well as the watersheds and subwatersheds which presented regions considerably similar in size and shape.

The qualitative comparison reinforced that the TIN model can generate good approximations to the potential water flows of real-world terrain surfaces as well as the quantification of their distribution over a region, which is an essential information in hydrological models. Therefore all the hydrological results generated in this work can be used in decision-making systems that support studies and researches of natural phenomena and environmental issues, although it is interesting to define specific measures in order to precisely validate the results.

Regarding the next steps to be taken in future works, one can include careful investigations of precise definitions about the concepts of a quantitative comparison between the TIN and regular grid hydrological structures. The comparison between drainage networks falls on a problem of similarity between graphs, which is not simple to be tackled since it depends on complex mathematical and computational properties related to graphs. In addition, the delineation of watersheds and subwatersheds are affected by the drainage networks, so that a comparison between these structures are also complicated, but can possibly be achieved with the use of polygon-matching methods.

Another relevant consideration to be made about the quantitative comparison is the fact that this work considered a regular grid generated from only one specific interpolation method. However, it is interesting to perform the same qualitative 
comparison with regular grids generated from different interpolation methods and also with distinct spatial resolutions in order to reasonably assess the methods that can produce regular grids presenting hydrological results closer to the results generated from the TIN model.

One also intends to include all the techniques described in this work as hydrologyspecific functionalities in the TerraHidro computational platform. In this case, the TerraHidro system would probably be the first GIS application to derive hydrological structures from TIN terrain models in the same way these structures are usually generated from regular grids, thus providing the end user a broad and rich number of possibilities for hydrological applications, so that the system will not be limited to just one type of terrain model structure.

Besides the issues referred to above, one can also consider the development of a specific procedure for an automatic correction of the problems present in the input data since a visual and manual correction process, as performed in this work, takes considerable time and effort to be accomplished. The input data correction is necessary to avoid inconsistencies in the hydrological results.

One more prospective concern includes the improvement of the computational times after a detailed analysis of all the procedures developed to process the TIN model, which can be achieved by performing further optimizations in the data structures and algorithms. Therefore an analysis of the time and space complexities of the algorithms is also an important issue to be considered.

It is also worth taking into consideration, as already pointed out in the beginning of this work, that future works will consider a thorough and detailed analysis of the methodology as well as the results from another work recently published (GUODONG et al., 2014), which presents a method that also generates and extracts drainage networks, watersheds and subwatersheds from accumulated flows defined over the TIN model. Furthermore, the results from that work will be compared to the results generated with the methodology proposed in the present work. 



\section{REFERENCES}

ABREU, E. S.; ROSIM, S.; RENNO, C. D.; OLIVEIRA, J. R. F.; JARDIM, A. C.; ORTIZ, J. O.; DUTRA, L. V. Terrahidro - A distributed hydrological system to delimit large basins. In: INTERNATIONAL GEOSCIENCE AND REMOTE SENSING SYMPOSIUM (IGARSS). Proceedings... Munich, Germany: IEEE, 2012. p. 546-549. 36,72

AGARWAL, P.; BERG, M. de; BOSE, P.; DOBRINDT, K.; KREVELD, M. J. van; OVERMARS, M. H.; GROOT, M. de; ROOS, T.; SNOEYINK, J.; YU, S. The complexity of rivers in triangulated terrains. In: 8TH CANADIAN CONFERENCE ON COMPUTATIONAL GEOMETRY (CCCG). Proceedings... [S.l.]: Carleton University Press, 1996. p. 325-330. ISBN 0-88629-307-3. 55

AGRAWAL, R.; AHMAD, N.; JAYPRASAD, P.; MAHTAB, A.; KUMAR, J. A. V.; PATHAN, S. K. Comparative evaluation of various algorithms for drainage extraction using CARTOSAT-1 stereo data. Agriculture and Hydrology Applications of Remote Sensing, v. 6411, p. 1-11, Dec 2006. 83

BARBALIC, D.; OMERBEGOVIC, V. Correction of horizontal areas in TIN terrain modeling - algorithm. In: 19TH ANNUAL ESRI INTERNATIONAL USER CONFERENCE. Proceedings... San Diego, CA, USA: Esri, 1999. Access in 12 jun. 2013. Available from: <http://proceedings.esri.com/library/userconf/ proc99/proceed/papers/pap924/p924.htm>. 2, 5, 23, 26

BERG, M. de; CHEONG, O.; KREVELD, M. v.; OVERMARS, M.

Computational geometry: algorithms and applications. 3. ed. Santa Clara, CA, USA: Springer-Verlag, 2008. 191-205 p. ISBN 978-3-540-77973-5. 1, 13, 14, 16, 17 , 18,19

BLACKBRIDGE. RapidEye - satellite imagery. 2008. 63

BOWYER, A. Computing Dirichlet tessellations. The Computer Journal, v. 24, n. 2 , p. $162-166,1981.14$

CAMARA, G.; SOUZA, R. C. M.; FREITAS, U. M.; GARRIDO, J. SPRING: Integrating remote sensing and GIS by object-oriented data modelling.

Computers \& Graphics, v. 20, n. 3, p. 395-403, May 1996. 72

CAMARA, G.; SOUZA, R. C. M.; PEDROSA, B. M.; VINHAS, L.; MONTEIRO, A. M. V.; PAIVA, J. A.; CARVALHO, M. T.; GATTASS, M. TerraLib: technology 
in support of GIS innovation. In: II BRAZILIAN SYMPOSIUM ON

GEOINFORMATICS (GEOINFO2000). Proceedings... Sao Paulo, SP, Brazil:

INPE, UNICAMP-DCC and Tecgraf PUC-RIO, 2000. 15

CIGNONI, P.; MONTANI, C.; SCOPIGNO, R. DeWall: A fast divide and conquer Delaunay triangulation algorithm in Ed. Computer-Aided Design, v. 30, n. 5, p. 333-341, Apr 1998. 14

COLLISCHONN, W. Simulacao Hidrologica de Grandes Bacias. PhD Thesis (PhD) - Universidade Federal do Rio Grande do Sul, Porto Alegre, Instituto de Pesquisas Hidraulicas, Dec 2001. 35

COLlisCHONN, W.; AllASIA, D.; SIlVA, B. C.; TUCCI, C. E. M. The MGB-IPH model for large-scale rainfall-runoff modelling. Hydrological Sciences Journal, v. 52, n. 5, p. 878-895, May 2007. 35

CORMEN, T. H.; STEIN, C.; RIVEST, R. L.; LEISERSON, C. E. Introduction to algorithms. 2. ed. [S.1.]: McGraw-Hill Higher Education, 2001. ISBN 0-07-013151-1. 52

EASTMAN, J. R. Idrisi32 Release 2: Guide to gis and image processing. Worcester, MA, USA, May 2001. v. 2. 19, 23

FORTUNE, S. A sweepline algorithm for Voronoi diagrams. Algorithmica, v. 2, p. $153-174,1987.14$

FOWLER, R. J.; LITTLE, J. J. Automatic extraction of irregular network digital terrain models. SIGGRAPH Computer Graphics, v. 13, n. 2, p. 199-207, Aug 1979. $1,8,12,36$

FREITAS, H. R. A.; ROSIM, S.; OLIVEIRA, J. R. F.; FREITAS, C. C. Drainage paths derived from TIN-based digital elevation models. In: XIV BRAZILIAN SYMPOSIUM ON GEOINFORMATICS (GEOINFO2013). Proceedings... Campos do Jordao, SP, Brazil: CCST, INPE and UNICAMP-IC, 2013. p. 31-42. $24,26,28$

GUERCIO, R.; SOCCODATO, F. M. GIS procedure for automatic extraction of geomorphological attributes from TIN-DTM. In: HYDROGIS 96: APPLICATION OF GEOGRAPHIC INFORMATION SYSTEMS IN HYDROLOGY AND WATER RESOURCES MANAGEMENT. Proceedings... Vienna: International Association of Hydrological Sciences (IAHS), 1996. p. 175-182. 55, 59 
GUIBAS, L. J.; KNUTH, D. E.; SHARIR, M. Randomized incremental construction of Delaunay and Voronoi diagrams. Algorithmica, v. 7, n. 1-6, p. 381-413, Jun 1992. 14

GUODONG, Q.; DANYANG, S.; ZHANGHUA, L. A new algorithm to automatically extract the drainage networks and catchments based on triangulation irregular network digital elevation model. Journal of Shanghai Jiaotong University (Science), v. 19, n. 3, p. 367-377, Jun 2014. 3, 87 INSTITUTO NACIONAL DE PESQUISAS ESPACIAIS - INPE. TerraView 4.1.0. Sao Jose dos Campos, SP, Brazil, 2010. 72

JARVIS, A.; REUTER, H.; NELSON, A.; GUEVARA, E. Hole-filled SRTM for the globe Version 4. 2008. International Centre for Tropical Agriculture (CIAT). 1, 36

JENSON, S. K.; DOMINGUE, J. O. Extracting topographic structure from digital elevation data for geographical information system analysis. Photogrammetric Engineering and Remote Sensing, v. 54, n. 11, p. 1593-1600, Nov 1988. 8, 36 JONES, N. L.; WRIGHT, S. G.; MAIDMENT, D. R. Watershed delineation with triangle-based terrain models. Journal of Hydraulic Engineering - ASCE, v. 116, n. 10, p. 1232-1251, Oct 1990. 1, 2, 7, 12, 14, 38, 41, 42, 44, 55, 59, 65

KIRK, R. E. Statistics: An introduction. 5. ed. [S.l.]: Thomson Wadsworth, 2008. 71

MCCOY, R. M. Automatic measurement of drainage networks. IEEE Transactions on Geoscience Electronics, v. 8, n. 3, p. 178-182, Jul 1970. 83 O'CALLAGHAN, J. F.; MARK, D. M. The extraction of drainage networks from digital elevation data. Computer Vision, Graphics, and Image Processing, v. 28, n. 3, p. 323-344, Dec 1984. ISSN 0734-189X. 8, 9, 36, 55

O'ROURKE, J. Computational geometry in C. 2. ed. New York, NY, USA: Cambridge University Press, 1998. ISBN 0521640105. 14

PETRIE, G.; KENNIE, T. J. M. Terrain modelling in surveying and civil engineering. Computer-Aided Design, Butterworth-Heinemann, Newton, MA, USA, v. 19, n. 4, p. 171-187, May 1987. ISSN 0010-4485. 1

PREFEITURA MUNICIPAL DE SAO JOSE DOS CAMPOS. Banco de dados 'Cidade Viva'. Rua Jose de Alencar, 123, 6o andar (paco municipal), 2003. 9, 64 
SILFER, A. T.; KINN, G. J.; HASSETT, J. M. A geographic information system utilizing the triangulated irregular network as a basis for hydrologic modeling. In: 8TH INTERNATIONAL SYMPOSIUM ON COMPUTER-ASSISTED CARTOGRAPHY. Proceedings... [S.1.], 1987. p. 129-136. 37, 38

SILVEIRA, R. I. Optimization of polyhedral terrains. PhD Thesis $(\mathrm{PhD})-$ Utrecht University, Jul 2009. 2, 5, 30

SILVEIRA, R. I.; OOSTRUM, R. van. Flooding countries and destroying dams. In: 10TH INTERNATIONAL WORKSHOP ON ALGORITHMS AND DATA STRUCTURES (WADS). Proceedings... Halifax, Canada: Springer-Verlag Berlin Heidelberg, 2007. (Lecture Notes in Computer Science, v. 4619), p. 227-238. ISBN 978-3-540-73948-7. 2, 5, 29, 30

SOILlE, P.; VOGT, J.; COLOMBO, R. Carving and adaptive drainage enforcement of grid digital elevation models. Water Resources Research, v. 39, n. 12 , p. 1366-1375, Dec 2003. 2, 5, 30

TSAI, V. J. D. Delaunay triangulations in TIN creation: an overview and a linear-time algorithm. International Journal of Geographical Information Systems, v. 7, n. 6, p. 501-524, 1993. 14

TSIROGIANNIS, C. P. Analysis of flow and visibility on triangulated terrains. PhD Thesis (PhD) — Eindhoven University of Technology, Nov 2011. 7, $13,38,39,40,42,44,45,55,59$

VOMACKA, T. Delaunay triangulation of moving points in a plane. 2008. University of West Bohemia, Pilsen. 15

WATSON, D. F. Computing the $n$-dimensional Delaunay tessellation with application to Voronoi polytopes. The Computer Journal, v. 24, n. 2, p. 167-172, 1981. 14

WIGMOSTA, M. S.; NIJSSEN, B.; STORCK, P. The distributed hydrology soil vegetation model. In: Mathematical models of small watershed hydrology and applications. Littleton, CO, USA: Water Resource Publications, 2002. chapter 2, p. 7-42. 35

YU, S.; KREVELD, M. v.; SNOEYINK, J. Drainage queries in TINs: from local to global and back again. In: 7TH INTERNATIONAL SYMPOSIUM ON SPATIAL DATA HANDLING. Proceedings... [S.1.], 1996. 55, 59 
ZALIK, B.; KOLINGEROVA, I. An incremental construction algorithm for Delaunay triangulation using the nearest-point paradigm. International Journal of Geographical Information Science, v. 17, n. 2, p. 119-138, 2003. 15

ZHANG, M. C.; CAMPBELL, J. B.; HARALICK, R. M. Automatic delineation of drainage basins within digital elevation data using the topographic primal sketch. Mathematical Geology, v. 22, n. 2, p. 189-209, Feb 1990. 59

ZHU, Y. J.; YAN, L. An improved algorithm of constrained Delaunay triangulation based on the diagonal exchange. In: 2ND INTERNATIONAL CONFERENCE ON FUTURE COMPUTER AND COMMUNICATION (ICFCC). Proceedings... Wuhan, China: IEEE, 2010. v. 1, p. 827-830. 2, 19, 21 
